\title{
Dimeric Cinchona alkaloids
}

\author{
Przemysław J. Boratyński
}

Received: 2 June 2014 / Accepted: 22 December 2014 / Published online: 15 January 2015

(C) The Author(s) 2015. This article is published with open access at Springerlink.com

\begin{abstract}
Nature is full of dimeric alkaloids of various types from many plant families, some of them with interesting biological properties. However, dimeric Cinchona alkaloids were not isolated from any species but were products of designed partial chemical synthesis. Although the Cinchona bark is amongst the sources of oldest efficient medicines, the synthetic dimers found most use in the field of asymmetric synthesis. Prominent examples include the Sharpless dihydroxylation and aminohydroxylation ligands, and dimeric phase transfer catalysts. In this article the syntheses of Cinchona alkaloid dimers and oligomers are reviewed, and their structure and applications are outlined. Various synthetic routes exploit reactivity of the alkaloids at the central 9-hydroxyl group, quinuclidine, and quinoline rings, as well as 3-vinyl group. This availability of reactive sites, in combination with a plethora of linker molecules, contributes to the diversity of the products obtained.
\end{abstract}

Keywords Quinine - Quinidine - Dimer · Trimer · Cinchona $\cdot$ Alkaloid

\section{Introduction}

The term alkaloid is used for many vastly different nitrogen heterocycles of mostly plant origin. Alkaloids are classified according to the heterocycle and the taxonomy of the species they were isolated from. The natural diversity of the alkaloids is further extended by the presence of numerous dimeric alkaloids (Fig. 1). Some dimers appear as byproducts

\section{P. J. Boratyński $(\bowtie)$}

Department of Organic Chemistry, Wrocław University of Technology, Wyspiańskiego 27, 50-370 Wrocław, Poland e-mail: przemyslaw.boratynski@pwr.edu.pl

URL: http://org.wch.pwr.edu.pl by coupling of a small portion of the monomers (e.g., salutadimerine), while others are the final products of biosynthesis (e.g., cephalostatin). Alkaloid dimers can exhibit biological activities unrelated to that of the corresponding monomer [1], such as in vitro anti-HIV and antimalarial properties of Michelleamine A [2], or fungicidal activity of Bismurrafoline B [3]. Natural alkaloid dimers of different symmetry and heterodimers were isolated (Fig. 1). Additionally, dimers of alkaloids can be synthesized in the laboratory giving rise to a virtually unlimited number of combinations [1].

The bark of various Cinchona species contains four major alkaloids, namely quinine (QN), quinidine (QD), cinchonidine (CD), and cinchonine (CN) (Fig. 2). These quinoline alkaloids are isolated on an industrial scale in multi-ton amounts. Their structures contain a central hydroxyl group as well as quinoline and quinuclidine rings. The individual alkaloids differ in the configuration at two crucial stereogenic centers (C-8 and C-9, Fig. 2). Quinine has been used for nearly four centuries to treat malaria. Although currently it is largely replaced by newer medicines, such as chloroquine (1947) and artemisinin (1970's), its therapeutic use is limited to drug-resistant strains. On the other hand, quinidine is often used to treat certain arrhythmias. Cinchona alkaloids are also employed in enantioselective synthesis (catalysts, ligands) and separation processes (resolving agents, solid phases, assays) [4]. To date, no dimeric alkaloid in this family has been isolated from a natural source. Nevertheless, many synthetic dimers were made exploiting a few reactive sites in the Cinchona alkaloids (Fig. 2).

These synthetic dimers were examined for their biological activities and applicability in asymmetric reactions. For the purposes of medicinal chemistry, the multiplication of the pharmacophore in the dimers could improve binding or cause crosslinking of the biological receptors. The transition from a monomeric to dimeric alkaloid molecule results in accumu- 
<smiles>COc1cc(C)cc2c(O)c(-c3cc(-c4c(O)cc(O)c5c4CC(C)N[C@H]5C)c(O)c4c(OC)cc(O)cc34)cc(-c3c(O)c(O)cc4c3CC(C)N[C@@H]4C)c12</smiles>

Michelleamine A (from Ancistrocladus korupensis)

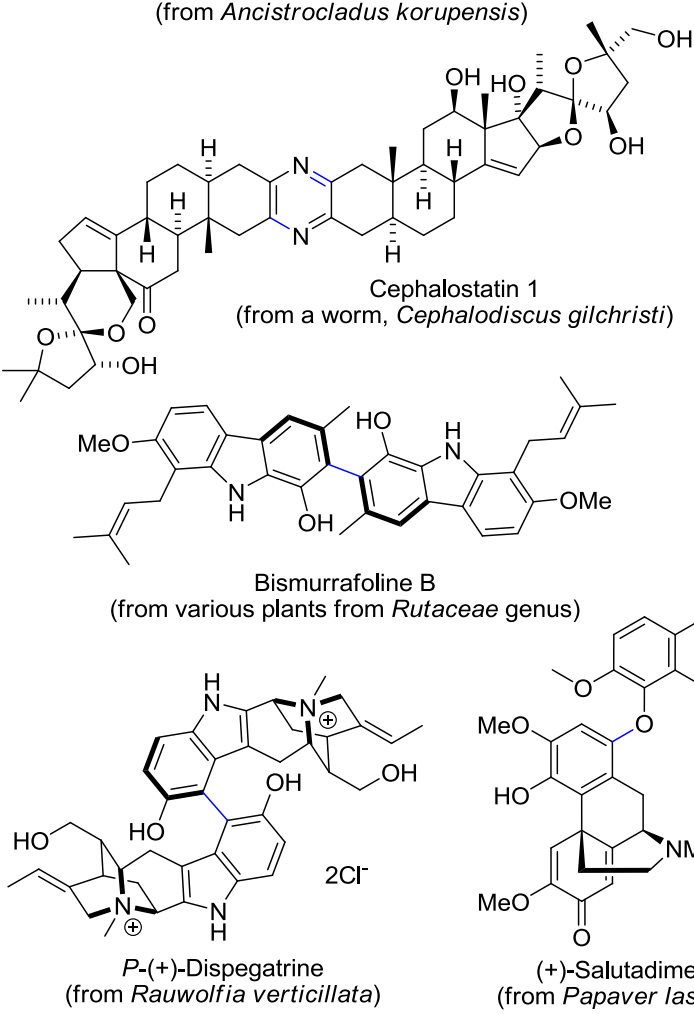
芒<smiles></smiles>
halostatin 1

Bismurrafoline $B$ ceae genus)

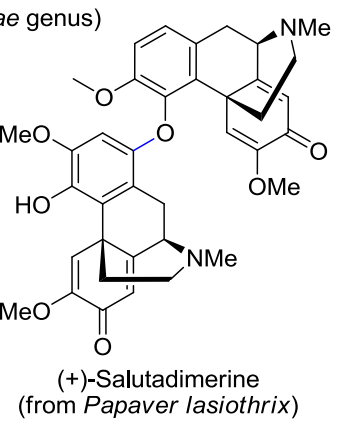

Fig. 1 Selected natural dimeric alkaloids

lation of functional groups confined within a limited space. These features as well as a $C 2$-symmetry are often present in effective asymmetric catalysts. Modifications of Cinchona alkaloids at the central 9-OH group and at the quinuclidine $\mathrm{N}-1$ atom led to the most effective dimeric catalysts and biologically active compounds.
For the purpose of this review, alkaloid derivatives are labeled with the corresponding alkaloid (QN, QD... cf. Fig. 2), 10,11-dihydroalkaloid (DHQN, DHQD...), or 9-epialkaloid descriptor ( $e \mathbf{Q N}, e \mathbf{Q D}$, etc. $)$ followed by a consecutive compound number. It has to be emphasized that some derivatization reactions were reported only for a single alkaloid, while others were exercised on a set of Cinchona alkaloids.

\section{Dimers connected at the central C-9 position}

The central 9-OH group offers an attractive site for modification (i.e., etherification and esterification reactions). Alternatively, the hydroxyl group can be replaced with a few other groups (e.g., $\mathrm{NH}_{2}$ ) and subsequently used for dimerization.

\section{9-Ether-linked dimers}

Dimers, in which the Cinchona alkaloid units are connected through 9-aryl ethers, represent a class of the most successful ligands for the Sharpless asymmetric dihydroxylation (AD) and related aminohydroxylation reactions (Figs. 3 and 4) [48].

The same ligands with tungstate catalyzed enantioselective sulfur oxidation with hydrogen peroxide [9]. Furthermore, numerous applications in metal-free catalysis emerged [4,10,11] and made Cinchona alkaloid derivatives included to the privileged chiral structures [12]. Examples of these asymmetric organocatalytic reactions include (Fig. 4): dichlorination [13], fluorination [14,15], opening of cyclic anhydrides (including dynamic kinetic resolution) [16,17], aldol [18] and Mannich-type reactions [19], various types of conjugate addition [20-23], cyanation of ketones [24], cyclopropanation [25], and nucleophilic substitution [26].

In dimeric Cinchona aryl ethers (Fig. 3), the linkers are either electron deficient heterocycles or anthraquinones. All of these compounds were obtained through aromatic nucleophilic substitution. Thus, the alkaloid units are mostly in the para position with the exception for few meta derivatives, but
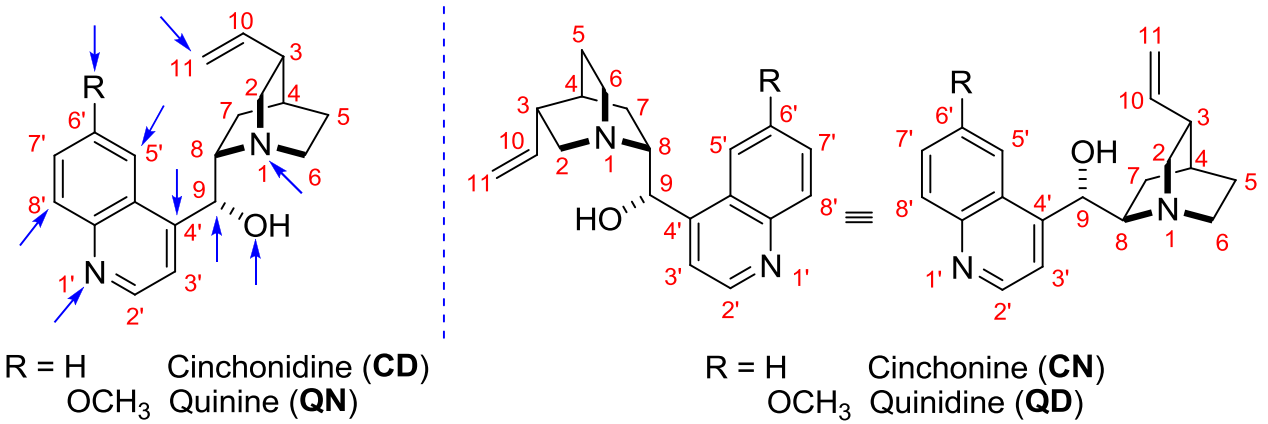

Fig. 2 Four major Cinchona alkaloids. Arrows mark the reactive sites used for dimerization 


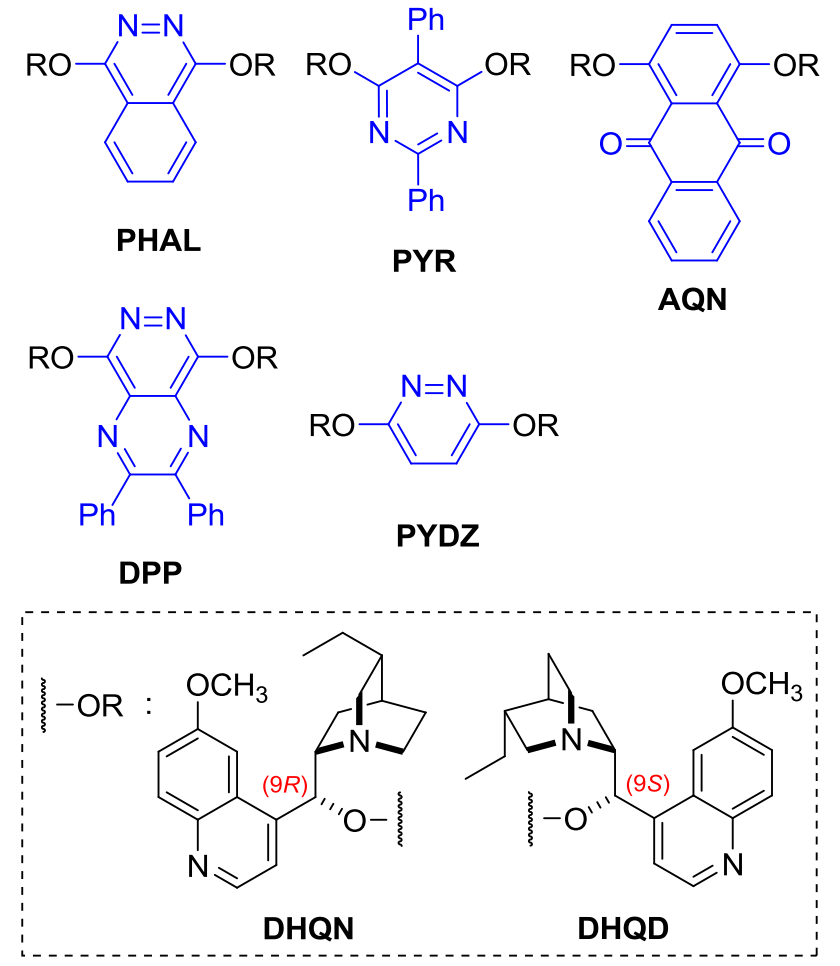

Fig. 3 Prominent linkers of dimeric aryl ethers and their common abbreviations

no ortho-diethers are known. The lack of such products arises from the reactivity of halo-aryls in the nucleophilic substitution, rather than steric interactions, since heavily substituted pyrimidine derivatives were obtained with relative ease.

\section{Phthalazine derivatives}

Up to 1992 there was an incremental progress in osmiumcatalyzed asymmetric dihydroxylation reactions, and promising results were obtained with monomeric Cinchona alkaloid derivatives in the role of ligands. Then, the discovery of dimeric phthalazine ether ligands (PHAL, 3) marked an enormous leap for asymmetric synthesis $[27,28]$. The respective dimers 3 were obtained from reactions of alkaloids with 1,4-dichlorophthalazine (2). The process required basic conditions and azeotropic removal of water with toluene [29]. In an alternative synthesis, the alkaloids were first deprotonated with $\mathrm{NaH}$ in DMF and subsequently treated with dichloride 2 [30]. This change in protocol often provided better preparative yields. Although $\mathbf{2}$ is commercially available, it can be efficiently obtained from phthalhydrazide (1), $\mathrm{PCl}_{5}$, and a catalytic amount of DMF (Fig. 5) [29].

Also, a stepwise protocol for the synthesis of unsymmetrical dimers was devised. Equimolar amounts of 1,4dichloro-phthalazine (2) and dihydroalkaloid gave reactive chloroderivative $\mathbf{4}$ that was used in a subsequent step to $9 \mathrm{O}$-arylate another alkaloid. The resulting quinidine- dihydroquinidine and quinine-dihydroquinine heterodimers 3 had a single vinyl group that was used to anchor the molecule to polymer supports using the radical addition of thiols (Fig. 6) [31-33].

A single reactivity averts crosslinking, and in the cases presented by the authors, also prevents significant distortion of geometry in the parent structure. The same approach was followed to obtain chiral stationary phase additive, by reaction of QD/DHQD-3 with octadecyl mercaptan [33]. The symmetrical phthalazine dimers $\mathbf{3}$ were also subject to many subsequent derivatization attempts. These include primarily immobilization, for example, direct copolymerization of quinine-based dimer with methacrylates [34], or copolymerization of more reactive alkaloid-derived acrylate DHQN-10 (Fig. 7) with styrene/divinylbenzene in suspension [35].

Apart from simple alkaloids, also their elaborate derivatives were dimerized with phthalazine [36]. Didehydroquinidine (QD-11, vide infra) was coupled in a Sonogashira reaction with various aryl halides to yield alkaloids with extended carbon scaffold QD-12a-b. Also, 11-iodinated didehydroalkaloid QD-12c was prepared by addition of iodine to the triple bond of didehydro-alkaloid followed by elimination of HI. These three compounds (QD-12a-c) were used to obtain respective phthalazine dimers QD-13a-c (Fig. 8). The yields of the dimerization step were similar to that of unmodified quinidine [37].

Under osmium-catalyzed asymmetric dihydroxylation conditions, the two native vinyl groups in Cinchona dimers QN-3, QD-3 are transformed to the corresponding tetraols. These products catalyze the AD reaction, although arguably [27] their effectiveness is inferior to DHQN-3 and DHQD3. Nevertheless, the polar character of these compounds was advantageous for reactions carried in special solvents, including ionic liquids, polyethylene glycol (PEG), and water. Thus, even more polar compounds were obtained by mono N1-quaternization with benzyl [30] or allyl bromide. The $N$-allyl ammonium salt QD-14 was then directly used in the AD reaction. It was transformed in situ to water soluble ammonium salt DHQD-15 having six hydroxyl groups, which facilitated recycling of the ligand through aqueous extraction (Fig. 9) [38]. Also, exhaustive quinuclidine $N$ alkylation was performed on phthalazine dimer DHQD-3. The obtained dimeric quaternary salts were not suitable for $\mathrm{AD}$ reactions, but were considered for phase transfer catalysis (PTC) [39].

Dimers of a different architecture were also obtained in a reaction of polyethylene glycols (PEG) with monomeric dihydroquinine ether DHQN-4 (Fig. 10). These immobilized soluble ligands DHQN-16a-c were still successful in aminohydroxylation reactions and could be recycled; however, significant catalyst loading was required [40].

Modification at the spacer unit required de novo synthesis of the dimers. The analogue of $\mathbf{2}$ with two pendant 


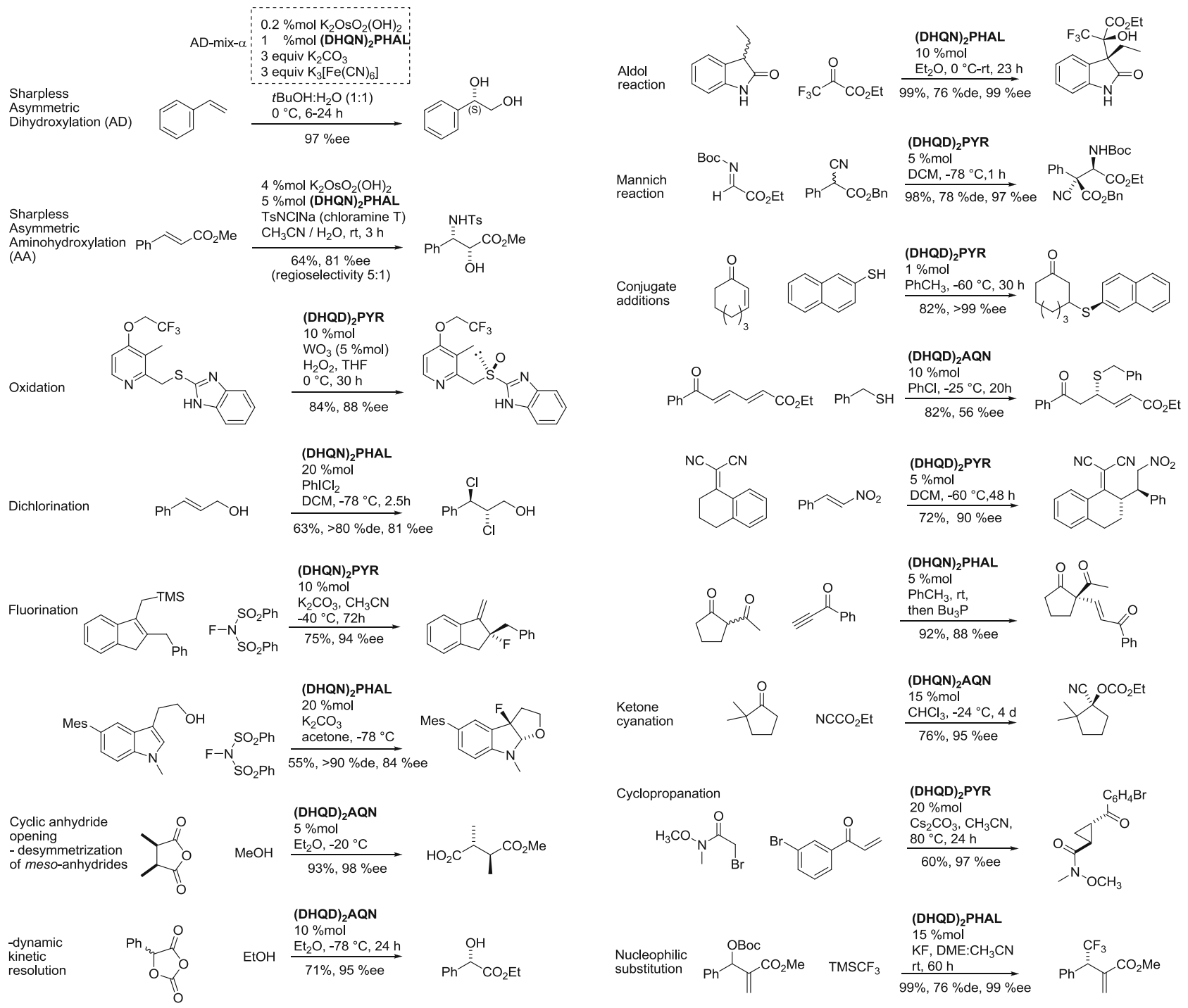

Fig. 4 Asymmetric reactions catalyzed by dimeric ethers of Cinchona alkaloids<smiles>O=c1[nH][nH]c(=O)c2ccccc12</smiles><smiles>Clc1nnc(Cl)c2ccccc12</smiles>

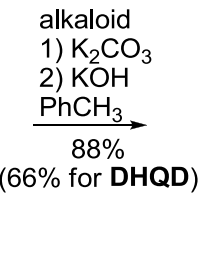<smiles>CCC1CC2CCC1CC2[C@H](CC1C2CCN1CC2CC)Oc1nnc(OC(c2ccnc3ccc(OC)cc23)c2ccnc3ccc(OC)cc23)c2ccccc12</smiles>

DHQN-3 (PHAL)

Fig. 5 Synthesis of $(\mathrm{DHQN})_{2} \mathrm{PHAL}$ dimer 


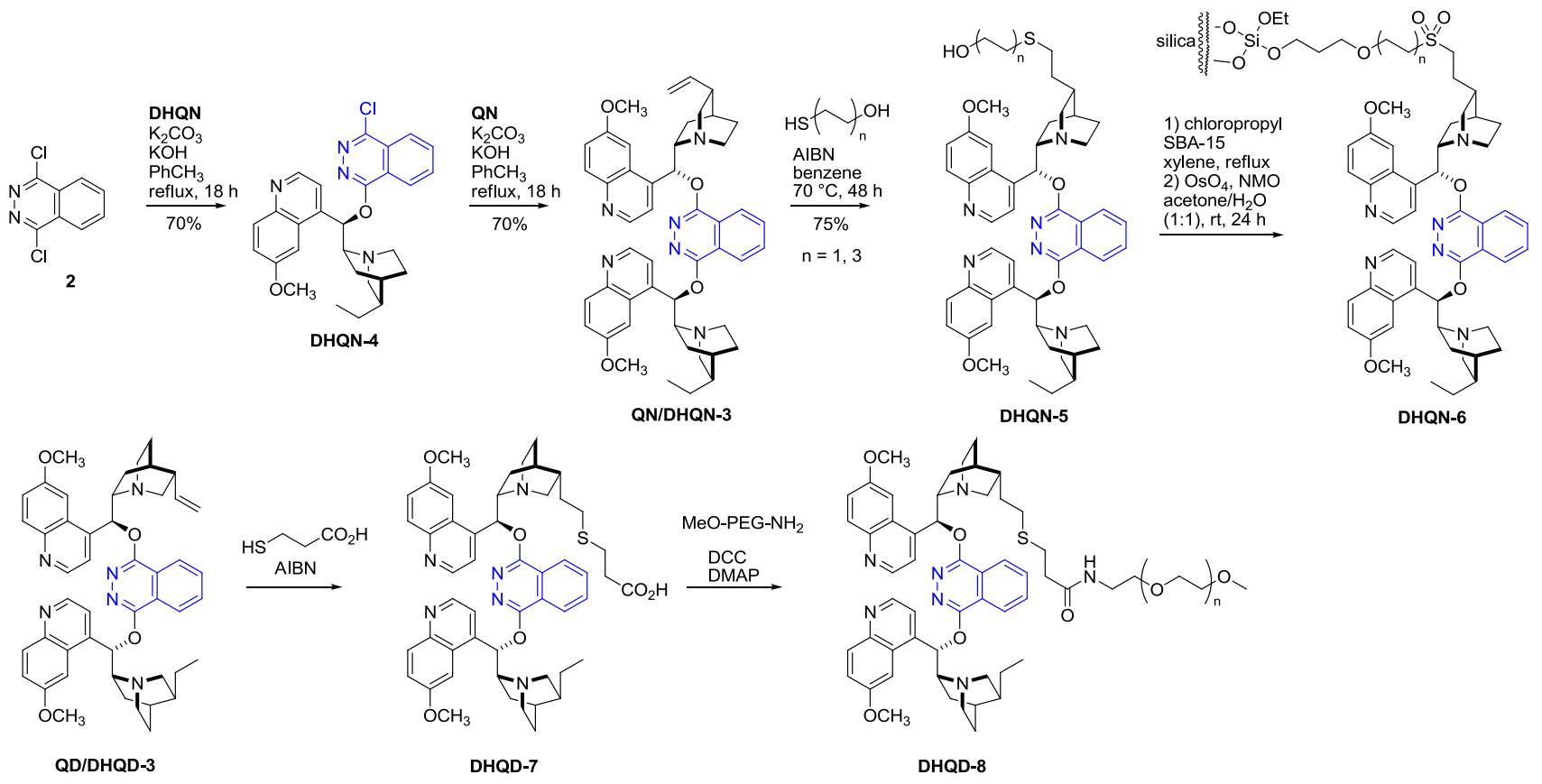

Fig. 6 Stepwise synthesis of PHAL-type heterodimers, and their immobilization

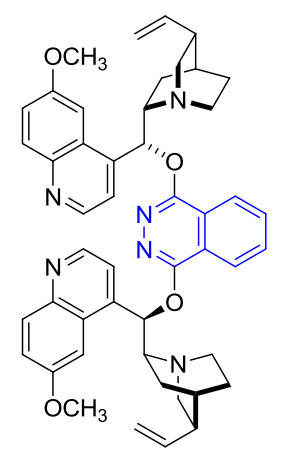

QN-3
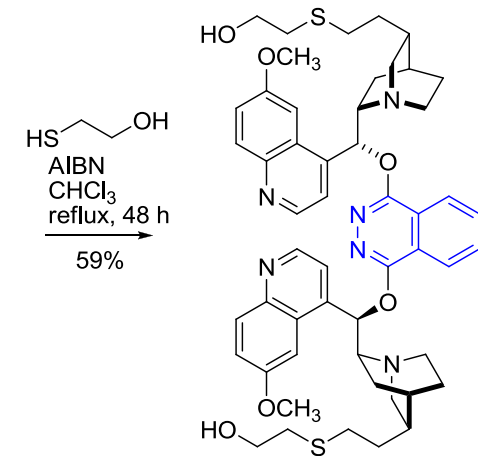

DHQN-9

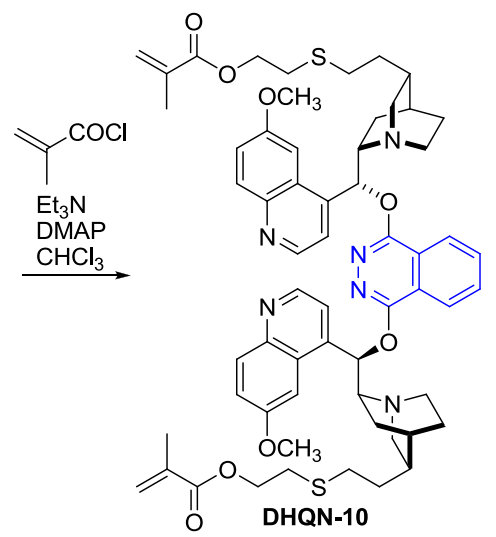

Fig. 7 PHAL-dimer derivative for copolymerization

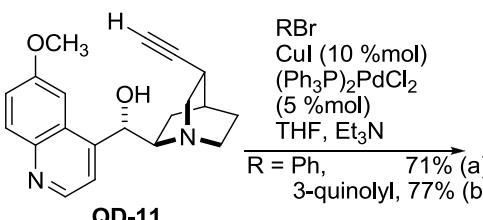

QD-11

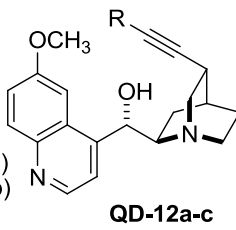

QD-12a-c

$\mathrm{I}_{2}$, morpholine, $\mathrm{PhCH}_{3}, 55^{\circ} \mathrm{C}, 10 \mathrm{~h}$

$\mathrm{R}=1,95 \%$ (c)

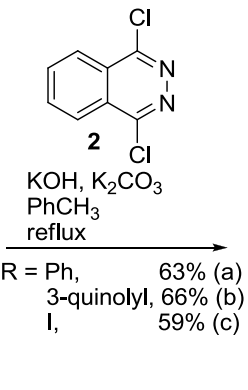

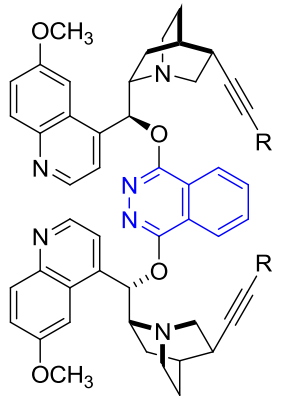

QD-13a-c

Fig. 8 PHAL-type dimers from modified Cinchona alkaloids 

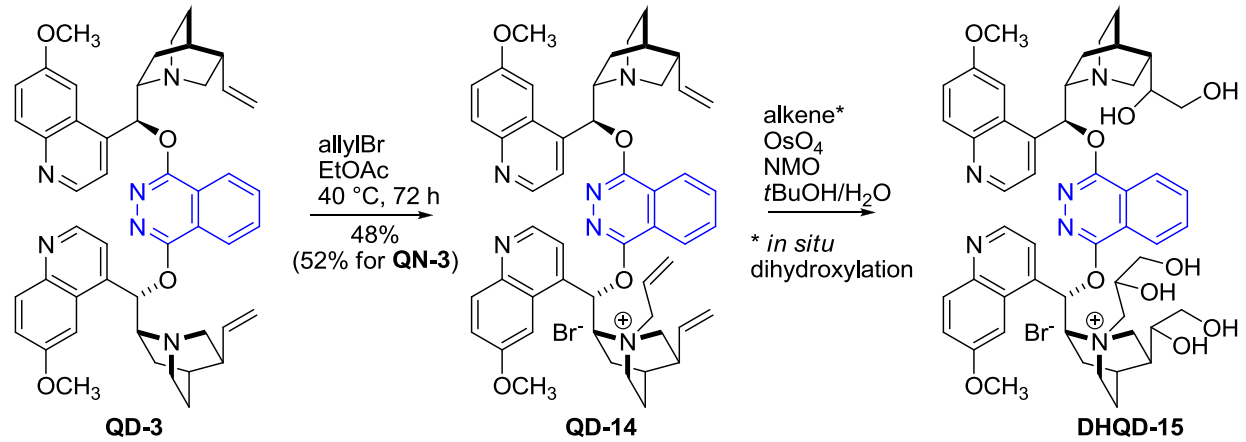

Fig. 9 Transformation of PHAL-dimer to hydrophilic species

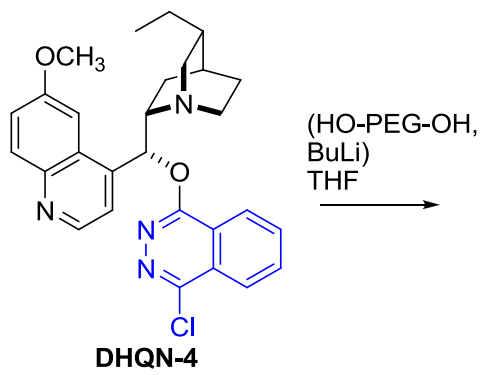

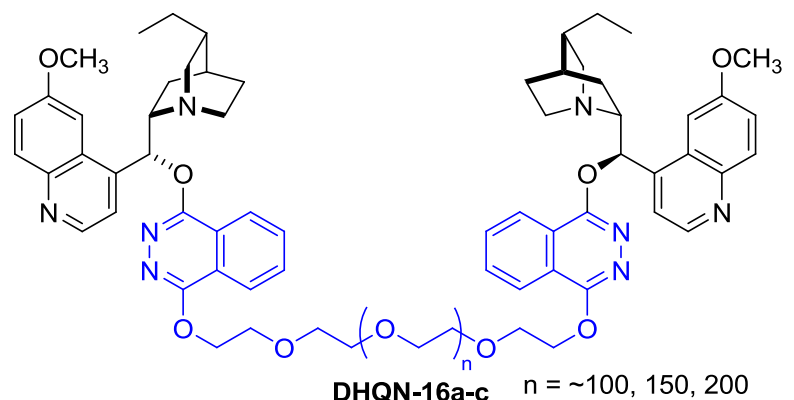

Fig. 10 Synthesis of alkaloid phthalazine ether-flanked PEG
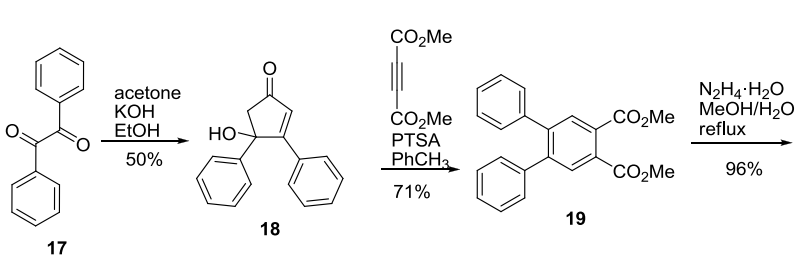

19
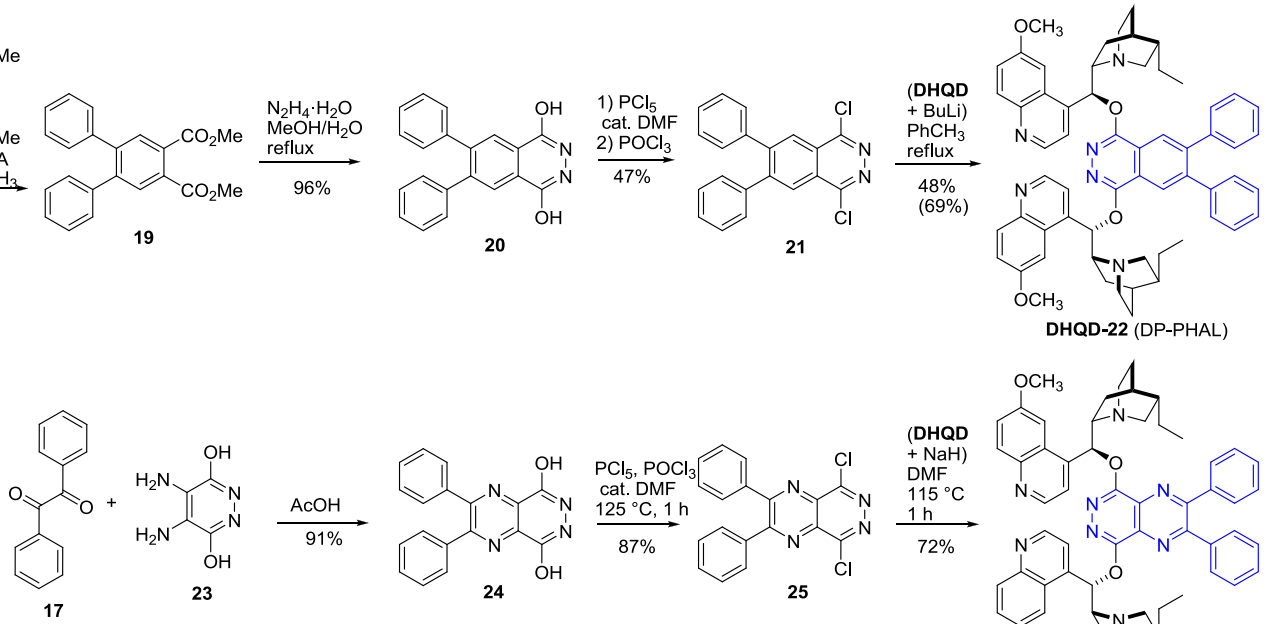

DHQD-22 (DP-PHAL)

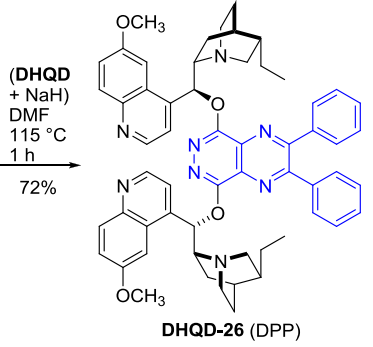

Fig. 11 Synthesis of DP-PHAL and DPP-type dimers

phenyl groups, 1,4-dichloro-6,7-diphenylphthalazine (21) was obtained in four steps (16\% total yield) from benzil (17). Coupling of $\mathbf{2 1}$ with both dihydroquinine and dihydroquinidine proceeded to DP-PHAL dimers DHQN-22 and DHQD-22 in 69 and $48 \%$ yield, respectively-i.e., by $20 \%$ lower than for the unsubstituted linker 2 [41]. A similar linker with two more nitrogen atoms incorporated into the planar ring system was also applied: 1,4-Dichloro-6,7-diphenylpyrazinopyridazine (25) [42] was coupled with two dihydro- quinidine units providing the respective DPP dimer DHQD26 (Fig. 11) [43].

Also, linkers with extended fused ring systems were applied. The synthesis of DHQD-32 by Corey involves two additional modifications. The alkaloid was first modified at the $6^{\prime}$-position of the quinoline ring (vide infra). Prior to coupling, dihydrocupreidine (DHQD-27), a derivative of quinidine with free $6^{\prime}$-hydroxy group, was $6^{\prime} O$-alkylated with a series of secondary alkyl bromides [44]. The reactive linker 


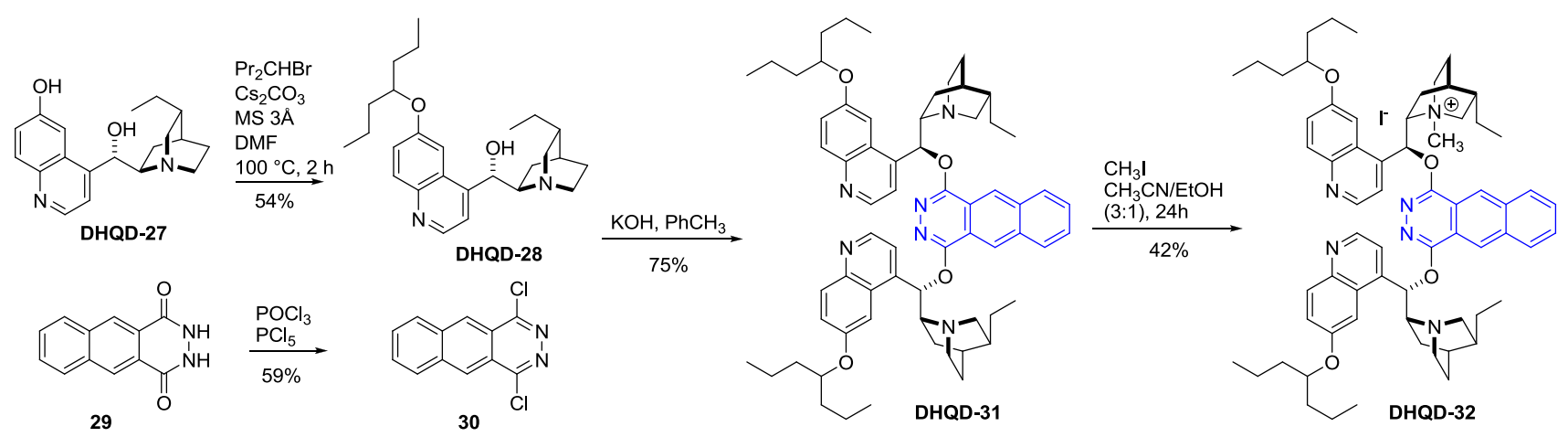

Fig. 12 Synthesis of a multiply modified PHAL-type heterodimer

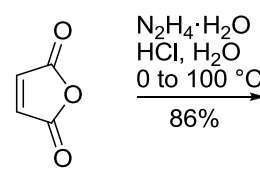

33

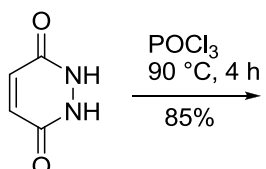

34

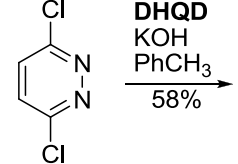

35

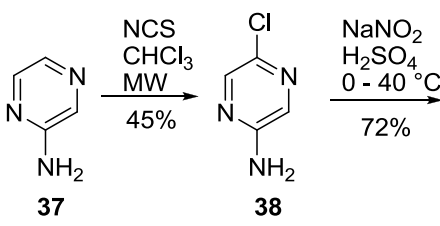<smiles>Oc1ncc(Cl)cn1</smiles>

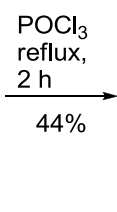<smiles>Clc1cnc(Cl)cn1</smiles><smiles>COc1ccc2nccc(C(Oc3ccc(O[C@H](CN4CC5CCC4CC5C)c4ccnc5ccc(OC)cc45)nn3)c3ccnc4ccc(OC)cc34)c2c1</smiles>

DHQD-36 (PYDZ)<smiles>COc1ccc2nccc(C(CN3CC4CCC3C4)Oc3cnc(O[C@@H]4CC5CCC(C5)N4)cc3[C@@H]3CCN4CCC3CC4C)c2c1</smiles>

DHQD-41

Fig. 13 Synthesis of PYDZ and pyrazine-linked dimeric ligands

molecule 30 was obtained analogously to phthalazine, starting from naphthalenedicarboxylic acid hydrazide and a mixture of $\mathrm{PCl}_{5}$ and $\mathrm{POCl}_{3}$ [45]. The coupling of quinidine derivative DHQD-31 with 1,4-dichlorobenzo[ $g]$ phthalazine afforded the dimer DHQD-31 in good yield. Subsequent partial N1-quaternization with methyl iodide concluded the synthesis of this highly diversified structure (Fig. 12). DHQD-32 provided a highly regioselective and enantioselective course of $\mathrm{AD}$ reaction of terminal isopropylidene groups in selected terpenoids [44].

\section{Pyridazine, pyrazine, and pyrimidine derivatives}

Shortly after introduction of phthalazine-type ligands by Sharpless, Cinchona dimers with two single-ring heteroaromatic linkers were applied by Corey's group [46]. These included pyridazine DHQD-36 (PYDZ) and pyrazine spacers DHQD-41. The coupling was accomplished by refluxing the respective 3,6- or 2,5-dichloro heterocycles $\mathbf{3 5}$ and $\mathbf{4 0}$ with dihydroquinidine in toluene in the presence of a base and the azeotropic removal of water. 3,6-Dichloropyridazine (35) not only is commercially available, but can also be obtained in a short and efficient synthesis [47]. On the other hand 2,5dichloropyrazine (40) is more challenging to obtain (Fig. 13) $[48,49]$.

Similarly to PHAL-dimers, a few modifications to the original PYDZ structure 36 were made. Double tethered derivatives of $\mathbf{3 6}$ are presented in the last section of this article. Dimer 36 was also partially quaternized with 9anthracenylmethyl group [50]. A pyridazine linker substituted with a short alkyl chain flanked with a terminal alkyne group was also obtained. The functionalized reactive dichloroheterocycle $\mathbf{4 4}$ was obtained via the sequential DielsAlder and retro-Diels-Alder reactions of dichlorotetrazine (42) and 1,7-octadiyne (43) in one pot. After the coupling of 44 with dihydroquinidine the terminal alkyne group of the dimer DHDQ-45 remained reactive in the copper-catalyzed Huisgen 1,3-dipolar "click" cycloaddition (CuAAC) [51]. Thus 1,2,3-triazoles were obtained with various azides including small molecules [52] and polymers (Fig. 14) [51].

Pyrimidine-based dimers 51 (PYR) constitute another important group of ligands, particularly useful in $\mathrm{AD}$ of 


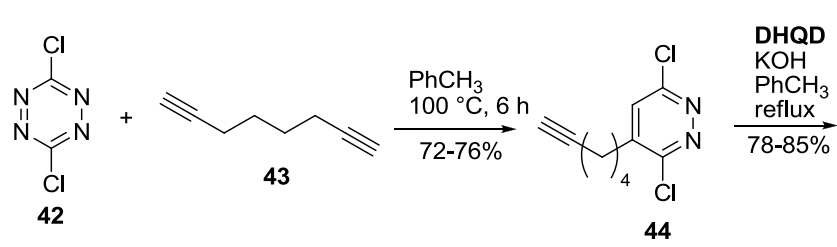

44
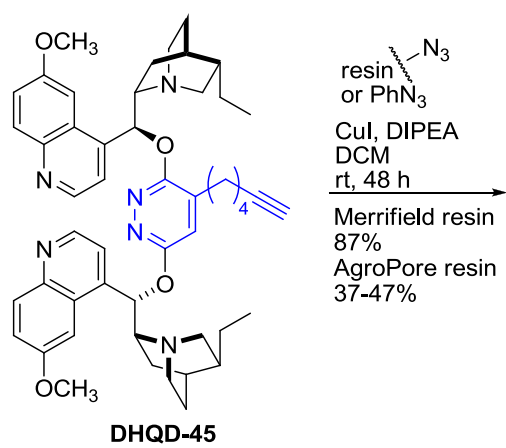

Fig. 14 Synthesis of PYDZ derivative reactive in "click" chemistry<smiles>CCOC(=O)C(CC)c1ccccc1</smiles>

47<smiles>N=C(N)c1ccccc1</smiles>

$\cdot \mathrm{HCl}$

48

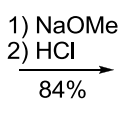

$\underset{84 \%}{\mathrm{HCl}}$

(2)
$\mathrm{HO}$<smiles>Oc1nc(-c2ccccc2)nc(O)c1-c1ccccc1</smiles>

49

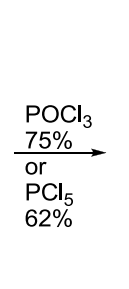

50

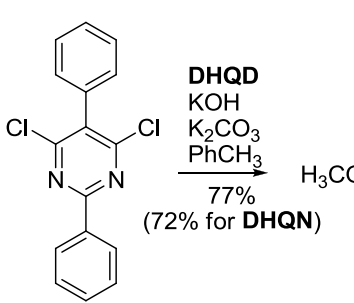

50

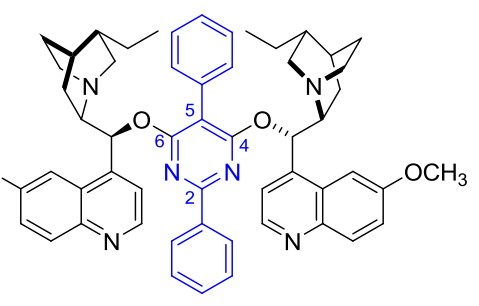

DHQD-51 (PYR)<smiles>CSc1nc(Cl)c(-c2ccccc2)c(Cl)n1</smiles>

52

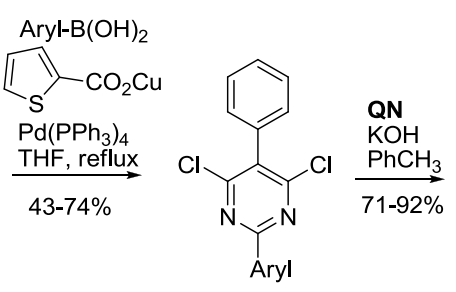

50a-d

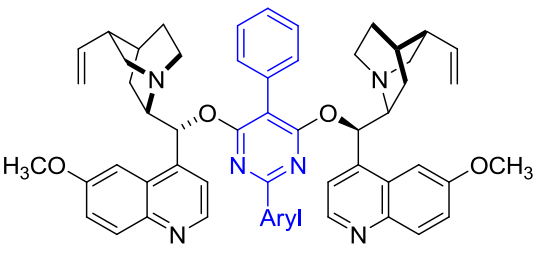

QN-51a-d

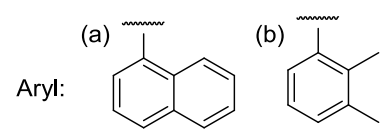

(c) (d) ${ }^{m}$

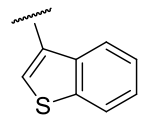

Fig. 15 Representative syntheses of PYR-type dimers

branched olefins. Their major distinction is that the alkaloid units are positioned meta instead of para to each other. The synthesis again relied on refluxing the dichloroheterocycle 50 with the alkaloid in the presence of a base in toluene and the azeotropic removal of water (Fig. 15). The reactive dichloride $\mathbf{5 0}$ was obtained in a two-step procedure starting from the condensation of adequately substituted diethyl malonate $\mathbf{4 7}$ and amidine $\mathbf{4 8}$. Subsequent reaction with either $\mathrm{POCl}_{3}$ or $\mathrm{PCl}_{5}$ gave the required reactive intermediate 50. An important feature of the pyrimidine scaffold is that 2- and 5- substituted derivatives are often easily accessible. Sharpless obtained dimers with pyrimidine linkers substituted at position 2 and 5 with combinations of phenyl and tert-butyl groups [53]. However, groups with greater steric demands at position 2 impede the formation of the dimer, and only monomeric alkaloid derivatives could be obtained from 2-CEt $3-5-t \mathrm{Bu}$-substituted pyrimidine [54]. In later reports, more differently 2 - and 5-substituted and unsubstituted pyrimidine dimers were mentioned [55]. The diversity of the products was further enhanced in a synthesis of several 2-aryl substituted dimers. The commercially available 4,6-dichloro-2-methylthio-5-phenylpyrimidine (52) reacted with a series of arylboronic acids in a Suzuki-type reaction. The obtained intermediates with quinine provided the dimers QN-51a-d in very good yields (Fig. 15) [56]. Also, an analogue of DHQN-51 substituted at the 2-position of the pyridine with 3,4,5-trimethoxyphenyl group was specifically designed for AD step in a synthesis of a natural product [57]. It was established that for applications in $\mathrm{AD}$ the presence of 2-tert-butyl is detrimental, while substitutions at 5-position are more tolerated [53]. Nevertheless, such tuning of the catalyst structure with bulky groups improved its performance in an asymmetric Feist-Bénary reaction [55,56].

Also, a related spacer with 1,3,4-triazine core was exploited. The synthesis was based on the reaction of inexpensive cyanuric chloride (53) with aniline to replace one of the reactive chlorides. Then, dichloride $\mathbf{5 5}$ was reacted with the prepared in situ quinine sodium salt in THF to provide the respective dimer $\mathbf{Q N - 5 6}$ in nearly quantitative yield. Although the authors used only 4-bromoaniline (54), they proposed that a diverse array of products could be obtained using different aniline or amine derivatives. Interestingly, an excess of quinine sodium salt with cyanuric chloride gave $C 3$-symmetric trimeric derivative QN-57 (Fig. 16). 


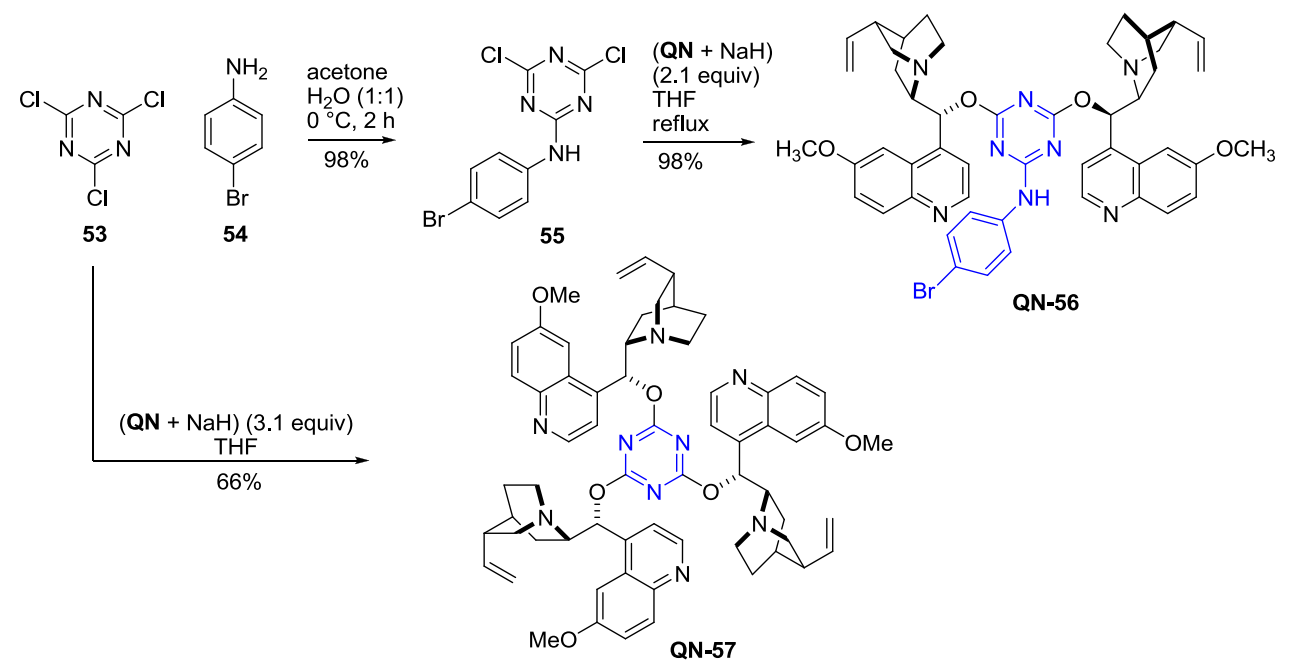

Fig. 16 Synthesis of 1,2,3-triazine-linked dimer and trimer

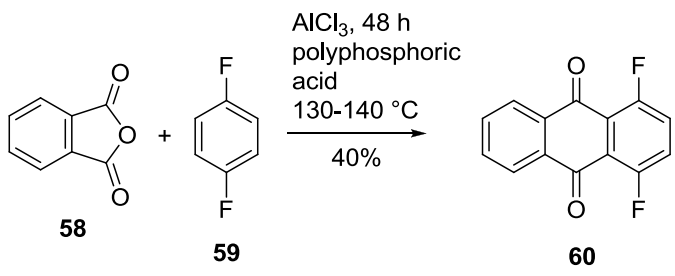

59

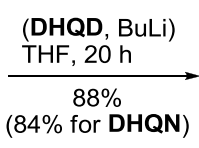

(84\% for DHQN)

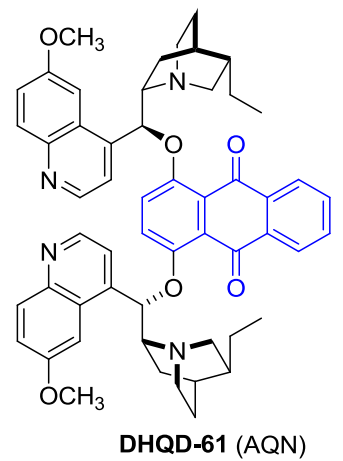

Fig. 17 Synthesis of AQN-type dimeric ligands

However, only dimer 56 showed promise in AD reactions [58].

\section{Anthraquinone derivatives}

Apart from the heterocyclic spacers, also the anthraquinone unit was extensively studied. 1,4-Difluroanthraquinone (60) was obtained in the Friedel-Crafts reaction of phthalic anhydride with $p$-difluorobenzene. For the coupling, dihydroalkaloid was converted in situ into a lithium salt with butyllithium, and then a reaction with difluorocompound $\mathbf{6 0}$ yielded the anthraquinone dimers (AQN, DHQN-61, DHQD-61) in very good yield (Fig. 17) [59]. These ligands are superior in AD of alkenes with aliphatic substituents.

Similarly to the phthalazine ligands, also a stepwise synthesis was devised. Consecutive reactions of $\mathbf{6 0}$ were carried out with alkaloid sodium salts in DMF. This approach, although lower in yield, allowed for the synthesis of heterodimers namely, alkaloid-dihydroalkaloid pair QN/ DHQN-61 suitable for immobilization (Fig. 18) [60].

Other immobilization attempts included addition of thiols to quinine and quinidine homodimers (QN-61 and QD-61)
[61]. Also, the spacer was modified to accommodate further transformations. 6-Bromoderivative of anthraquinone 66 was obtained from 4-bromo- $o$-xylene (64) in a four-step synthesis. The coupling with the alkaloid afforded 6-bromo derivative of AQN-dimer DHQD-67, which was reactive in a Suzuki coupling with TBS-protected 4-hydroxyphenylbor onic acid. The silyl ether was cleaved, and the obtained phenol group was exploited to obtain a series of derivatives 6973 (Fig. 19) [62]. Among these were linear polystyrene [63], silica gel supported material, polyethylene glycol derivatives [62] including a tetramer DHQN-73 formed from tethered dimeric quinine units [64].

\section{9-Alkyl ethers}

Dimeric alkaloid alkyl ethers constitute a much less studied group of compounds. Their synthesis is, however, straightforward and involves the Williamson etherification of an alkali metal alkaloid salt and the respective alkyl dihalide. For xylylene-linked dimers, all three isomers: ortho [65], meta [66], and para [67,68] were reported or mentioned in the literature. The shortest known link was made 

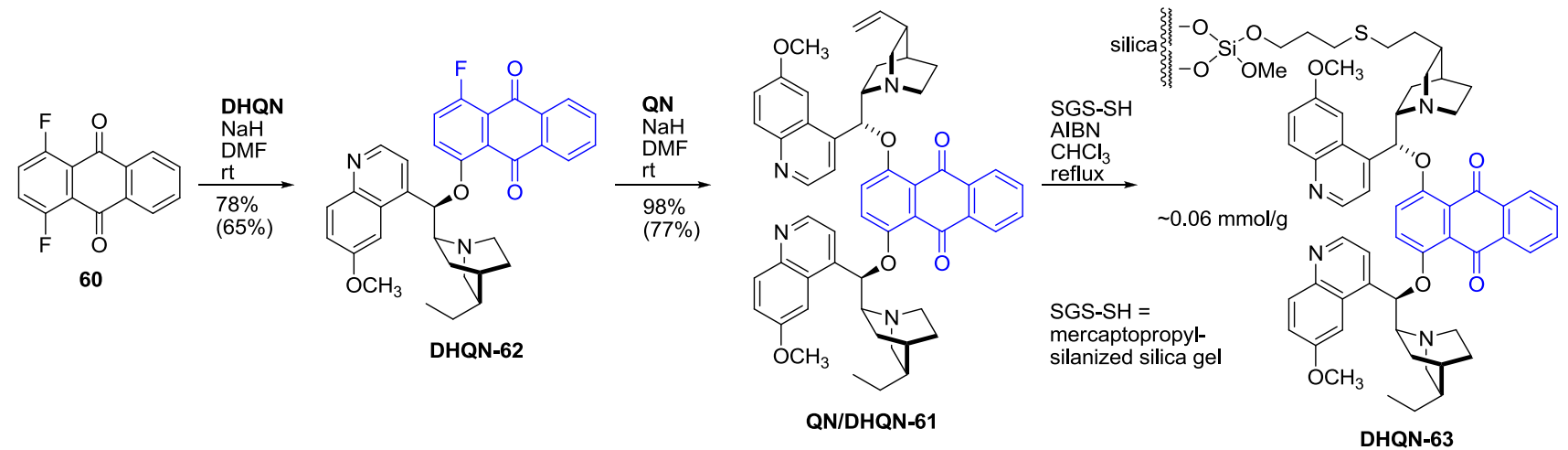

Fig. 18 Stepwise synthesis of AQN-type heterodimers, and their immobilization

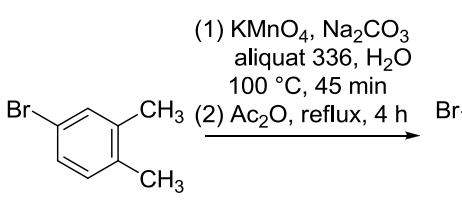

64

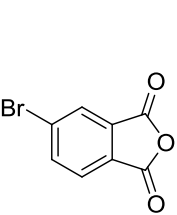

65

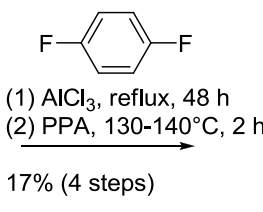<smiles></smiles>

66

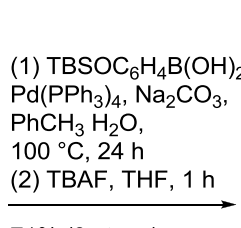

$71 \%$ (3 steps)<smiles>CCC(C)c1ccnc2ccc(OC)cc12</smiles>

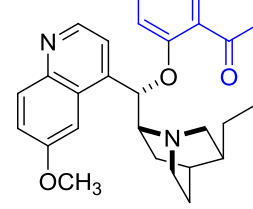

$\mathrm{R}=\mathrm{H}, \mathrm{DHQD}-\mathbf{6 8}$<smiles>C1CC2CCC(C1)C2</smiles><smiles>[Li]</smiles> 

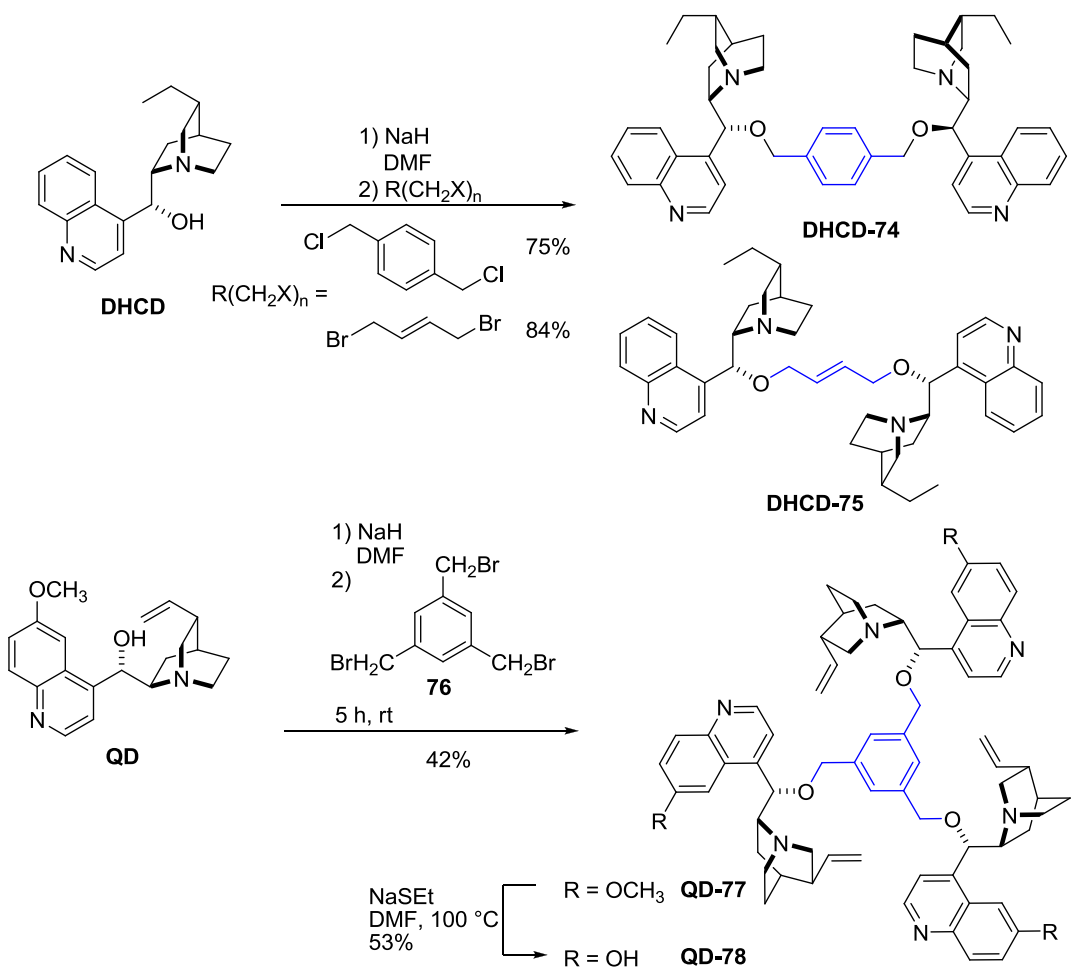

Fig. 20 General synthesis of dimeric and trimeric alkaloid alkyl ethers
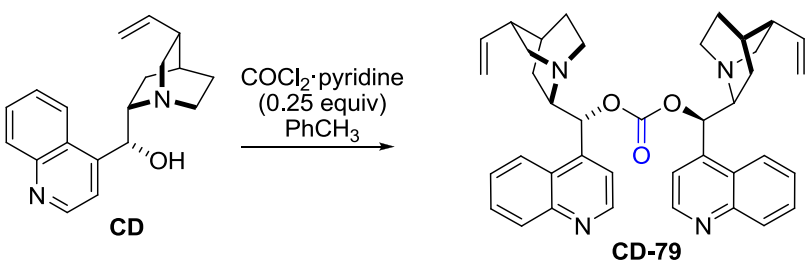

Fig. 21 Synthesis of dimeric alkaloid carbonate

acid chlorides and the alkaloids. Usually the transformation was carried in the presence of a base such as triethylamine [71] and sometimes with catalytic amounts of 4dimethylaminopyridine (DMAP). The yields, when reported, were above $70 \%$. The dimers were also obtained in a one-pot procedure, where the starting diacids were first transformed to the corresponding chlorides with thionyl or oxalyl chloride and subsequently coupled with the alkaloids. Alternatively the dicarboxylic acids were activated with a carbodiimide (e.g., EDC); however, this milder method often resulted in poor yields [72]. Following one of these general methods, a relatively large array of dimeric esters was synthesized (Figs. $22,23)$. Links were formed from simple aliphatic diacids with 3-10 carbon atoms [71,72], as well as those with ether (German patent No. DE237450, 1909) and disulfide bonds $[72,73]$.

Dimers linked with spacers having unsaturated bonds, aromatic rings, and bicyclic scaffolds were also obtained, mostly using corresponding acid chlorides for coupling. Cinchona dimers with all of the isomers of benzenedicarboxylic acid were obtained, namely terephthalic [71], isophthalic [74], and $o$-phthalic esters 86, 88, and 90, respectively. Dimeric esters with linkers incorporating heteroatoms were obtained, starting from dichlorides of pyridine 2,6-dicarboxylic acid [75], 2,2'-diselenodibenzoic acid [76], and ferrocene 1, 1'dicarboxylic acid (Fig. 24) [77]. Also, a C3-symmetric trimer QN-95 was formed in a reaction of trimesic acid chloride with quinine (Fig. 25) [71].

A few of the esters were tested in AD reaction. Some, like the ferrocene-linked dimer DHQN-91 provided only moderate enantioselectivity ( $<61 \%$ ee). In the group of simple diesters, hexadionate DHQN-81b turned out an effective catalyst (92\%ee) in contrast to malonate. The most attractive catalytic properties were found for the terephthalate ligand (DHQN-86, >98\%ee), which in AD of certain substrates outperformed the classic PHAL-type ligand DHQN-3 $[71,78]$. On the other hand, isophthalic ester, while still effective in $\mathrm{AD}$, provided worse enantioselectivity than terephthalates in all the cases studied [74]. In a later study, improved results in $\mathrm{AD}$ and $\mathrm{AA}$ reactions were achieved for isophthalates and a series of analogous pyridine-linked dimers $\mathbf{8 9}$ [79].

Diversity in the linker structure was also introduced by subsequent modifications of an already dimeric molecule. In the reaction of fumaroyl dichloride with cinchonine and cinchonidine, the respective dimeric esters $\mathbf{C N - 9 6}$ andCD- 


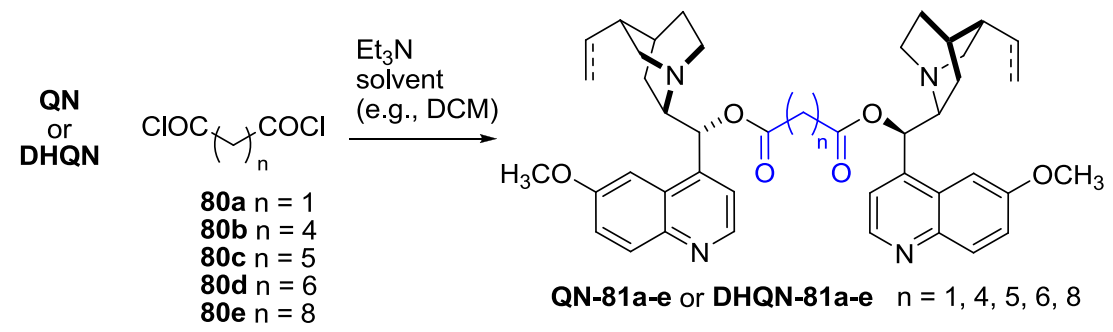

Fig. 22 General synthesis of alkaloid dimeric esters<smiles>[R16]O[Na]</smiles><smiles>NOC(=O)CCCSSCCCC(=O)O[Na]</smiles><smiles>O=C(CCCCSSCCCCC(=O)OS(=O)(=O)S)O[N+](=O)[O-]</smiles><smiles>C=CC1CC2CCN1C(C(OC)c1ccnc3ccc(OC)cc13)C2</smiles>

Fig. 23 Linear spacers with heteroatoms

96 were formed in 78-86\% yield [80]. The reactivity of the activated double bond was further exploited in a Diels-Alder reaction with cyclopentadiene and isoprene (Fig. 26). Consequently, a set of cyclohexene- and bicyclic dicarboxylic acid esters 97 and 98 were obtained. These were, however, not viewed as target compounds, instead Cinchona alkaloids were used as chiral auxiliaries for the Diels-Alder reaction, and the esters were cleaved afterward. The transformations using dimers provided much improved enantioselectivity compared to that of monomeric esters also used in the study (94-99\% vs. 6-93\%ee) [80]. Interestingly, an inverted sequence of Diels-Alder and acylation reactions was also explored. The enantiomeric diacids, adducts of anthracene and fumaric acid, were converted to acid dichlorides and reacted with dihydrocinchonidine. The products were assayed in asymmetric dihydroxylation of stilbene giving 52-85\%ee. Better results were obtained for ester of $11 R, 12 R$ configuration DHCN-94b than the $11 S, 12 S$ diasteromer DHCN-94a (Fig. 24) [81].
Another strategy for the synthesis of dimeric esters of Cinchona alkaloids was to first obtain monomeric esters with linker precursors that could be coupled later. In one such approach esters with a terminal alkyne group 99a-d and azido-esters $\mathbf{1 0 0 a}-\mathbf{d}$ of various chain length were formed by carbodiimide coupling. Using these components a combinatorial library of dimers 101aa-dd was obtained in a copper-catalyzed 1,3-dipolar 'click' cycloaddition (Fig. 27). In products $\mathbf{1 0 1}$ the linker units are non-symmetric. Since the esterification step is performed separately, well-defined heterodimers could also be obtained. The products were studied for inhibition of P-glycoprotein, and optimum linker length was established at 6 methylene groups at each side of the triazole unit (as in QN-101cc) [82].

Few examples of dimers with more elaborate and functional linkers relevant to supramolecular and coordination chemistry were obtained. One such scaffold incorporated a chiral crown ether. Ether $\mathbf{1 0 2}$ with two hydroxymethyl groups was modified with phthalic anhydride, and the resulting diacid was converted to acid chloride 104. Subsequent reaction of $\mathbf{1 0 4}$ with cinchonine gave dimeric ester $\mathrm{CN}-105$ in $78 \%$ yield (Fig. 28). Although the authors saw potential in the product for phase transfer catalysis, they did not develop the idea further [83].

In a study on the derivatization of annulenes, a one-pot procedure to introduce one or two alkoxycarbonyl groups to nickel dibenzotetraaza[14]annulene complex (106) was developed. The sequence of reactions involved FriedelCrafts acylation with oxalyl chloride, followed by decarbonylation and alkoxydehydrohalogenation [84]. The chirality of the product was assured with nonracemic alcohols, including quinine. The respective dimeric ester QN-107 was formed in $20 \%$ yield (Fig. 29). This result is similar to that obtained for other explored alcohols [85].

A series of dimeric carbamates was obtained according to two general protocols $[73,86]$. In the first one, the alkaloid was treated with diisocyanate derived from the corresponding diamine. This process was highly efficient furnishing the dimers in 64-87\% yield; however, it was only attempted for the commercially available diisocyanates. Alternatively, a two-step procedure was used: First the alkaloid was converted to an active carbonic ester QN-111 in a reaction with nitrophenyl chloroformate (110). Then, reaction of an excess 


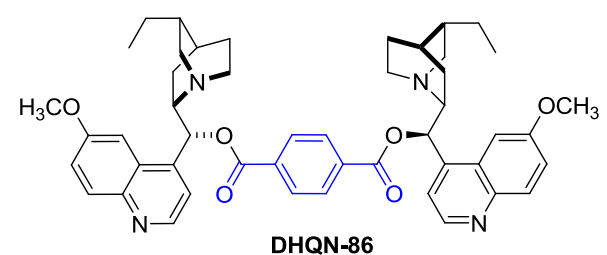

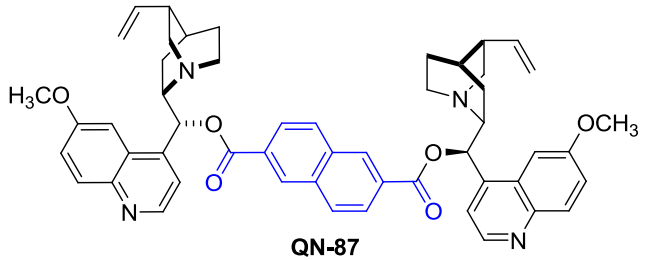<smiles></smiles>

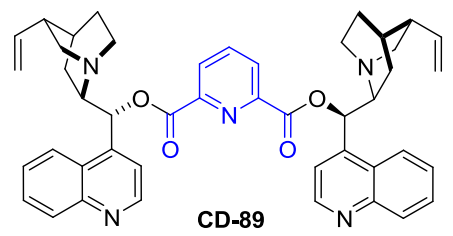<smiles>C=CC1CC2CCN1CC2[C@H](OC(=O)c1ccccc1C(=O)OC(c1ccnc2ccc(OC)cc12)[C@@H]1CC2CCCN1C2)c1ccnc2ccc(OC)cc12</smiles>

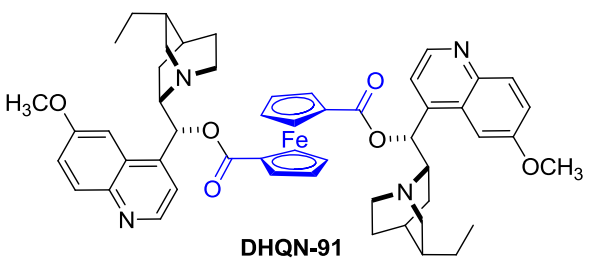

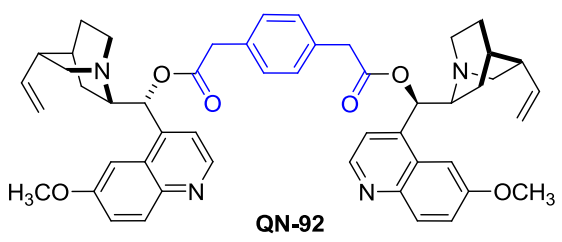

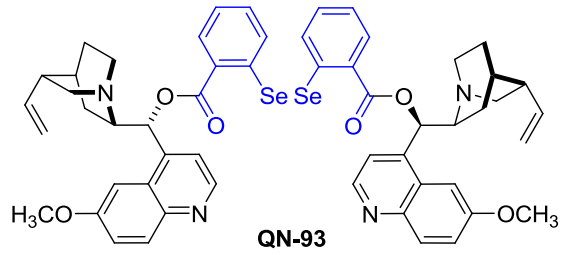

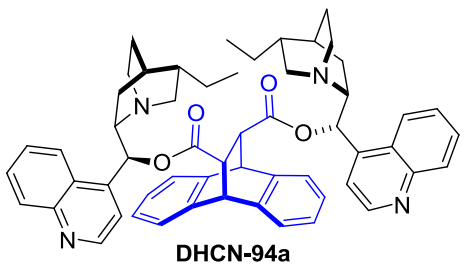

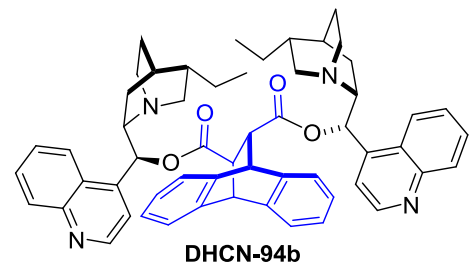

Fig. 24 Representative dimer structures with aromatic spacers

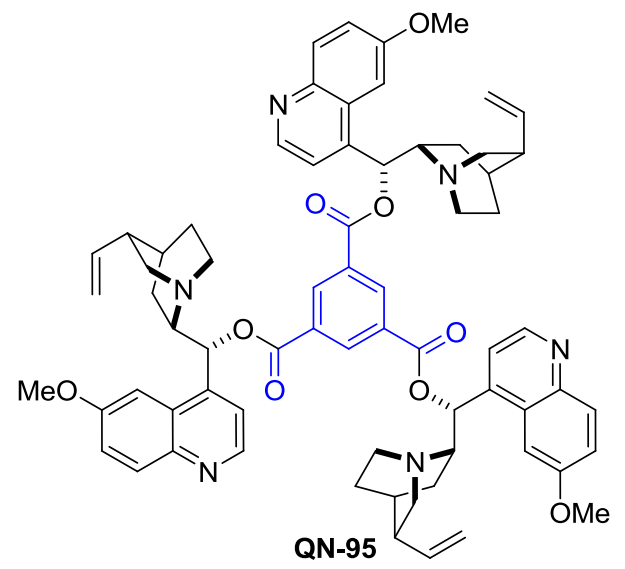

Fig. 25 Trimeric Cinchona alkaloid ester

active ester with diamine gave the corresponding dimeric carbamates in 35-66\% yield (Fig. 30). In both approaches the lowest yields were noted for 1,2-diamine derivatives [86].

Some of the Cinchona carbamates were subsequently immobilized on silica (through addition of thiols to the vinyl group) and used as chiral solid phases for anion exchange chromatography of amino acids. The use of dimers, compared to monomeric carbamates, led to longer retention times of the analytes, however, offered no improvement in the enantioselectivity of the separation. Out of the dimeric modifiers of silica gel, the best separation of enantiomers was achieved using the 1,3-adamantyl-linked QN-114 [86].

The esters and carbamates of Cinchona alkaloids were also examined as antimalarial agents. They were tested in vivo against drug-resistant Plasmodium falciparum and for the inhibition of PfCRT ${ }^{\mathrm{CQR}}$ — a multidrug resistance transporter protein. Dimers linked with $\left(\mathrm{CH}_{2}\right)_{8}$ ester $\mathbf{Q N - 8 0 e}$ and $\left(\mathrm{CH}_{2}\right)_{6}$ carbamate $\mathbf{Q N - 1 1 2 d}$ (both with the same number of bonds separating two quinine units) turned out to be the most effective [73]. 


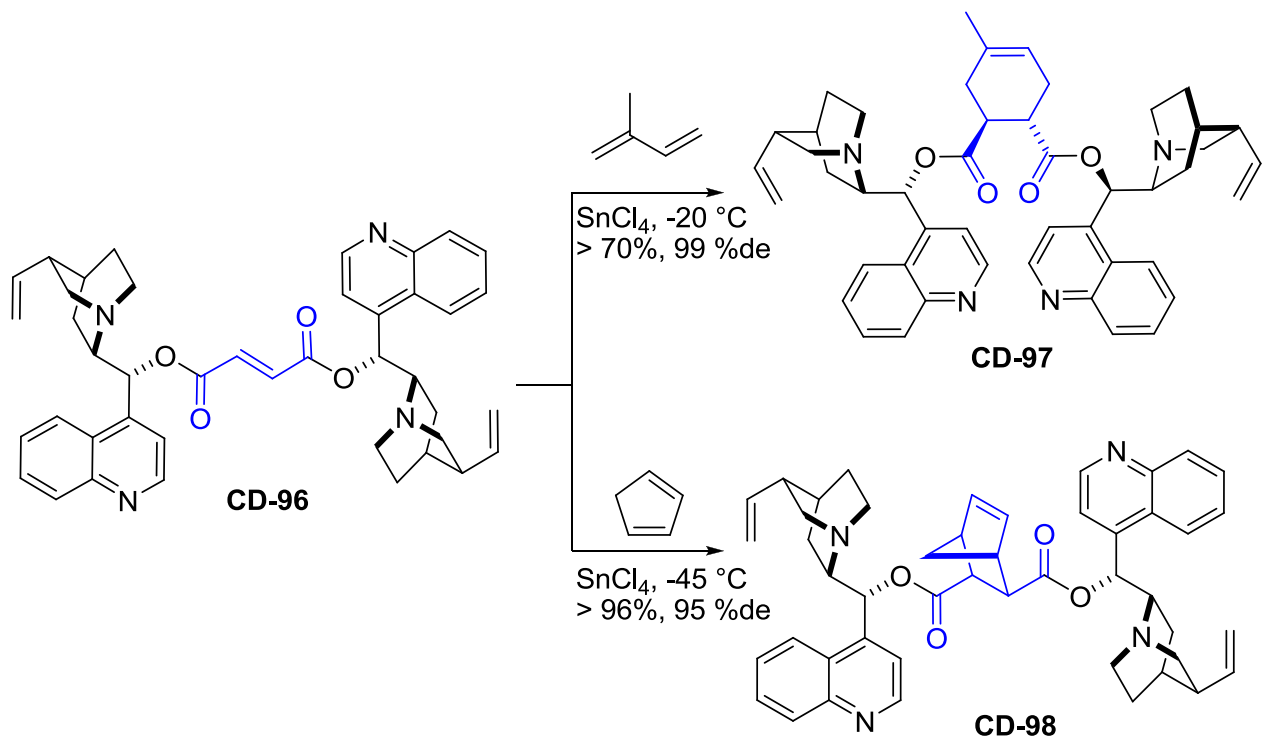

Fig. 26 Cyclic linkers obtained in diastereoselective Diels-Alder reaction

9-Nitrogen-linked dimers

The 9-hydroxyl group in the Cinchona alkaloids can be replaced with an amino group with inversion of configuration at C-9 [87]. The alkaloid undergoes a Mitsunobu reaction with an azide source (e.g., HN3, diphenylphosphoryl azide) to give 9-epi-azido-alkaloid 116. This azide is subsequently reduced with triphenylphosphine to amine $\mathbf{1 1 7}$ (Staudinger reduction). This sequence was often followed as a one-pot procedure. Alternatively, the azido-alkaloid $\mathbf{1 1 6}$ can be obtained in an $\mathrm{S}_{N} 2$ reaction from alkaloid methanesulfonate and $\mathrm{NaN}_{3}$ in DMF. The primary amino group of the 9-epi-9-deoxy-9-aminoalkaloid $\mathbf{1 1 7}$ was then subjected to reactions with various activated linker molecules producing a series of derivatives including amides, ureas, imines, etc. Reaction of oxalyl and isophthalic acid dichlorides with 9-aminocinchonine $e \mathbf{C N}-117$ gave the respective dimers $e \mathbf{C N}-118$ and $e \mathbf{C N - 1 1 9}$ [88]. Dimeric amide $e \mathbf{C N}$ 120 was obtained in a reaction of aminoalkaloid and sebacic acid applying HATU, a standard peptide coupling reagent (Fig. 31) [73]. The oxalyldiamide $e \mathbf{C N}-118$ was tested as catalyst in the diethylzinc addition to benzaldehyde providing only moderate enantioselectivity, though the ee was improved compared to monomeric amide analogs [88].

Gawroński and coworkers obtained dimeric imides $e$ DHCN-122 and $\mathbf{1 2 4}$ in the reaction of Cinchona alkaloids with 1,2,4,5-benzenetetracarboxylic anhydride (121) and 1,4,5,8-naphthalenetetracarboxylic anhydride (123). The initially formed dimeric amide was cyclized to imide $e \mathbf{D H C N}$ 122 by heating of the reaction mixture with acetic anhydride. The linear alignment induced by the linker resulted in observable conformer populations, in which the alkaloid units were oriented either syn $(C)$ or anti $(S)$ (Fig. 32) [89].
Without an additive, the conformer populations were equal. However, the ratio varied in response to carboxylic and dicarboxylic acids [90]. The prevalent conformation and their equilibria were studied by circular dichroism (CD) [89] and ${ }^{1} \mathrm{H}$ NMR spectroscopy. In addition, an analytical system composed of $e$ QD-124 and bromophenol blue allowed for visual identification of $\alpha$-hydroxycarboxylic acids, as well as their spectrophotometric estimation. This indicator displacement technique allowed for the determination of tartaric acid in wine as well as some differentiation of enantiomers [91].

A bis-alkaloid-thiourea $e \mathbf{C N}-\mathbf{1 2 6}$ was obtained in a reaction of $\mathrm{CS}_{2}$ with 9 -aminocinchonine $e \mathbf{C N}-\mathbf{1 1 7}$ in $51 \%$ yield [92]. Dimer $e \mathbf{D H Q N - 1 2 6}$ with the same linker was furnished in a reaction of aminoalkaloid $e \mathbf{D H Q N}-\mathbf{1 1 7}$ with thiocarbonyldiimidazole (TCDI) in DCM in $73 \%$ yield (Fig. 33) [93]. However, other researchers obtained merely $2 \%$ of the identical product $e$ DHQN-126 using THF as a solvent [94]. The monomeric alkaloid thioureas are now wellestablished bifunctional organocatalysts in asymmetric synthesis [95], while the dimers $\mathbf{1 2 6}$ were found to provide higher level of enantioselectivity in a few cases including dynamic kinetic resolution (DKR) of racemic azalactones (91\%ee with $e$ DHCD-126) [93] and cooperative sulfonation of enones (76\%ee with $e \mathbf{Q N - 1 2 6 )}$ [96]. Also dimer $e \mathbf{D H Q N}$ $\mathbf{1 2 6}$ had pronounced cytotoxic and cytostatic effects on SHSY5Y and HL-60 tumor cell lines [94].

A set of ferrocene spacers was applied for the dimerization of $e \mathbf{D H Q N}-117$ (Fig. 35). Diamide $e \mathbf{D H Q N - 1 2 9}$ was formed in a reaction between the aminoalkaloid and $1,1^{\prime}$ bisfluorocarbonylferrocene, while analoguous urea derivative $e \mathbf{D H Q N}-131$ was obtained from 1,1'-bisisocyanatoferrocene. 1,1'-Bis(chlorocarbonyl)ferrocene was treated with $\mathrm{KSCN}$ in acetone giving a reactive $1,1^{\prime}$-bisisothiocyanatocar 


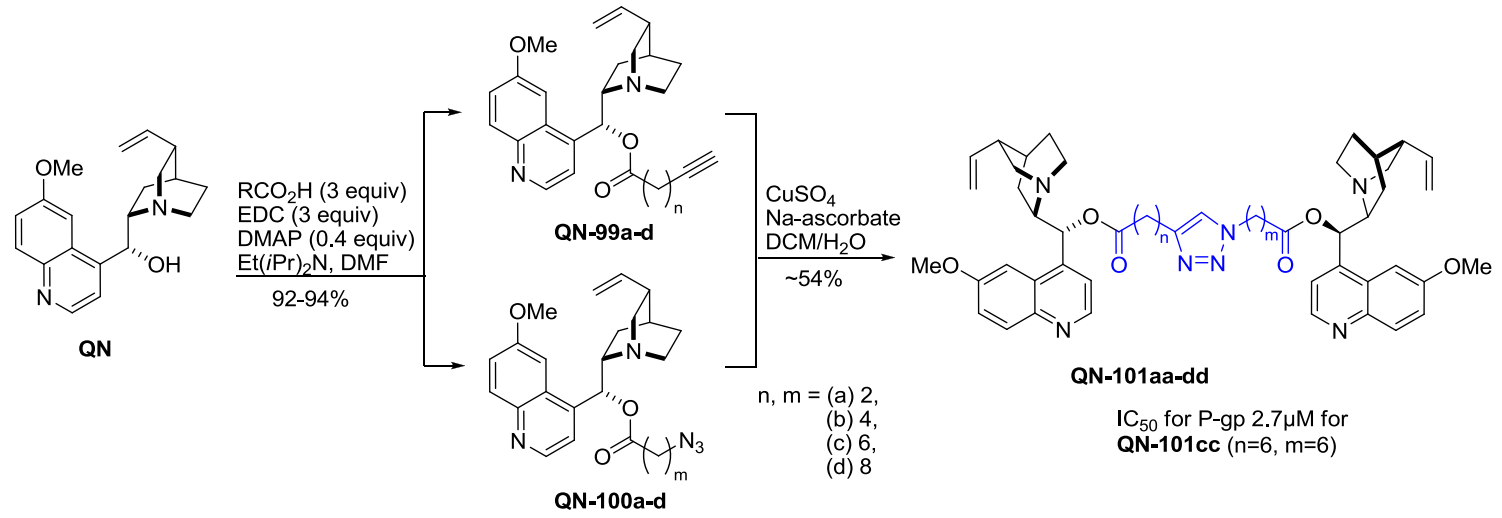

Fig. 27 "Click" assembly of quinine esters
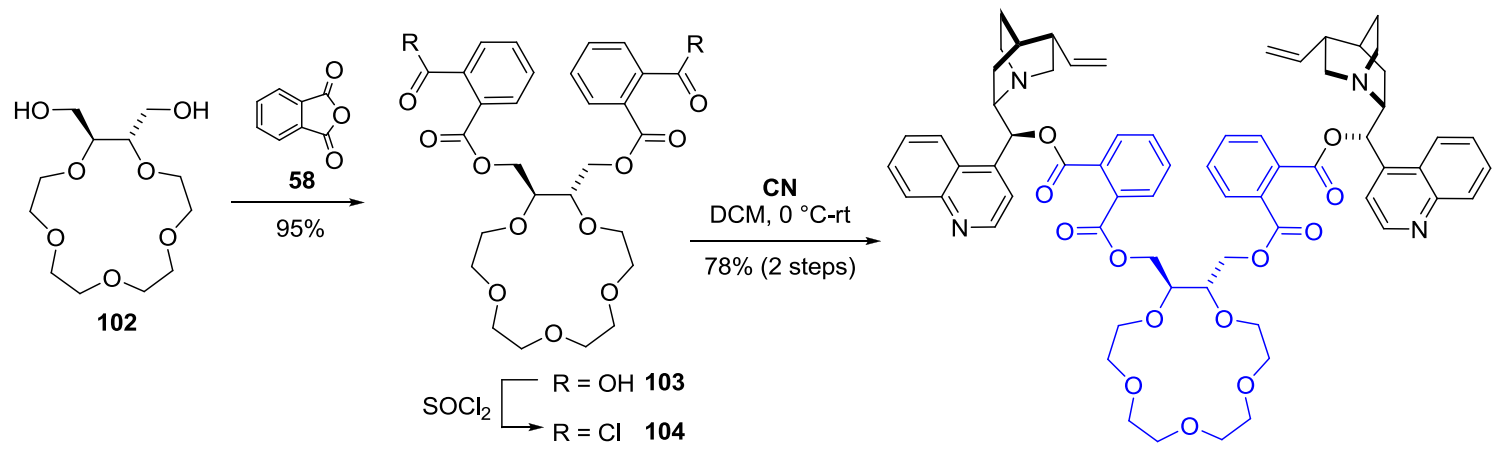

Fig. 28 Synthesis of crown ether-linked dimer

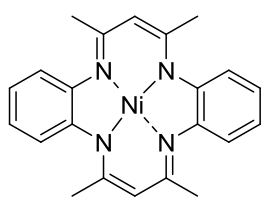

106

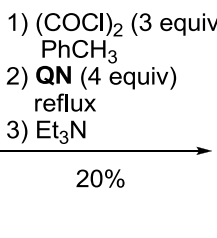

$\mathrm{PhCH}_{3}$

QN (4 equiv)

reflux

$20 \%$

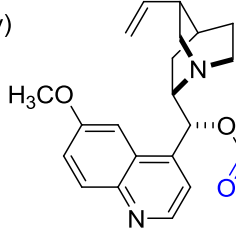

-<smiles>C=C1C2CCN(C2)C1c1ccnc2ccc(OC)cc12</smiles>

QN-107

Fig. 29 One-pot synthesis of dibenzotetraaza[14]annulene-linked dimer

bonyl intermediate 132. After solvent exchange, reaction of $\mathbf{1 3 2}$ with aminoalkaloid $e$ DHQN-117 provided dimeric acylthiourea $e \mathbf{D H Q N - 1 3 3}$. The reported yields for the urea derivatives were very poor $(2 \%)$ and were attributed to numerous acylation and polymerization reactions, and tedious purification processes. In the same work, the authors obtained benzenetricarboxylic acid triamide $e \mathbf{D H Q N}-\mathbf{1 3 4}$ in $87 \%$ yield from the respective acid trichloride (Fig. 36). The dimers, in particular $e \mathbf{D H Q N - 1 2 9}$ with the shortest ferrocene link, exhibited pronounced cytotoxic and cytostatic effects on HepG2, SH-SY5Y, HL-60, and MCF-7 human tumor cells [94].

Dimeric guanidine derivative $e$ DHCN-127 was also noted. It was obtained from 9-amino-dihydrocinchonine $e \mathbf{D H C N}$ 117 and $\mathrm{BrCN}$ (Fig. 34). This dimer was tested in a diastere- oselective Henry reaction, but it was not more selective than other catalysts [97].

Amides of squaric acid (1,2-dihydroxy-cyclobuten-3,4dione) with Cinchona alkaloid units were recently shown to be effective hydrogen bond donors in organocatalysis [98]. The synthesis of dimeric squaramides was straightforward. It entailed mixing 9-aminoalkaloid $\mathbf{1 1 7}$ and squaric acid dimethyl ester (135a) in methanol for $24 \mathrm{~h}$, while the products 136 precipitated in nearly quantitative yields (Fig. 37) [99]. The dimers were exploited in dynamic kinetic resolution of azalactones. Unlike the monomeric Cinchona squaramides, the dimers do not form self-associates [99]. Nevertheless, these two classes of compounds have different application scope, and often unsymmetrically substituted monomeric squaramides were superior catalysts [100]. 
400

Mol Divers (2015) 19:385-422
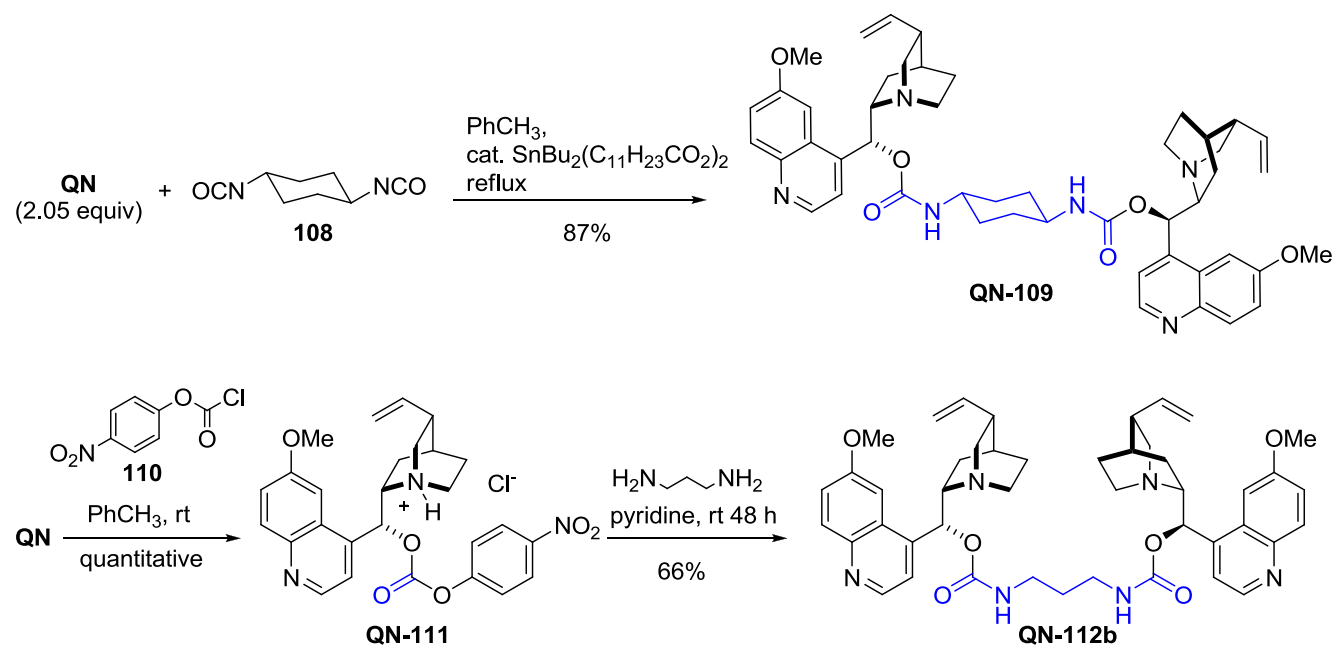<smiles>[R]OC(=O)NC(C)NC(=O)O[R]</smiles><smiles>[R]OC(=O)NC1CCCCC1NC(=O)O[R]</smiles>
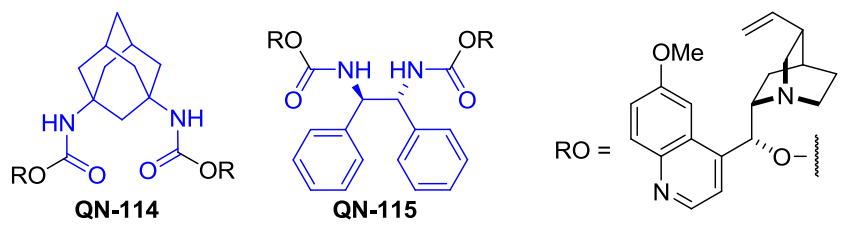

Fig. $30 \quad 9 O$-Alkaloid carbamate dimers and their synthesis<smiles>C=CC1CC2CCN1C2[C@H](O)c1ccnc2ccc(OC)cc12</smiles>

$\mathrm{HN}_{3}, \mathrm{PPh}_{3}$, DEAD, THE<smiles>C1CCCCC1</smiles>

1) $\mathrm{MsCl}$,

$\mathrm{Et}_{3} \mathrm{~N}, \mathrm{THF}$

2) $\mathrm{NaN}_{3}, \mathrm{DMF}$<smiles>C=CC1C2CCN1C([C@H](N)c1ccnc3ccc(OC)cc13)C2</smiles>

1) $\mathrm{PPh}_{3}$,

WHF

2) $\mathrm{H}_{2} \mathrm{O}$<smiles>C=CC1CC2CCN1C2C(N)c1ccnc2ccc(C#N)cc12</smiles>

eQN-117

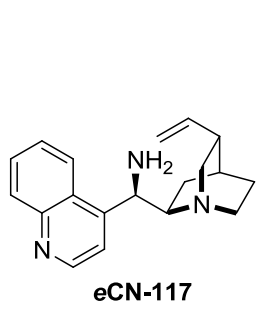

$(\mathrm{COCl})_{2}$

$32 \%$
$\mathrm{Et}_{3} \mathrm{~N}, \mathrm{DCM}$
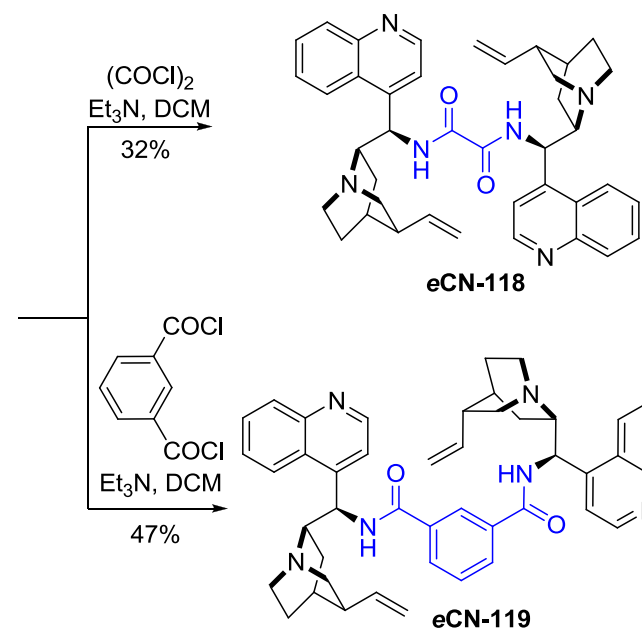

eCN-118
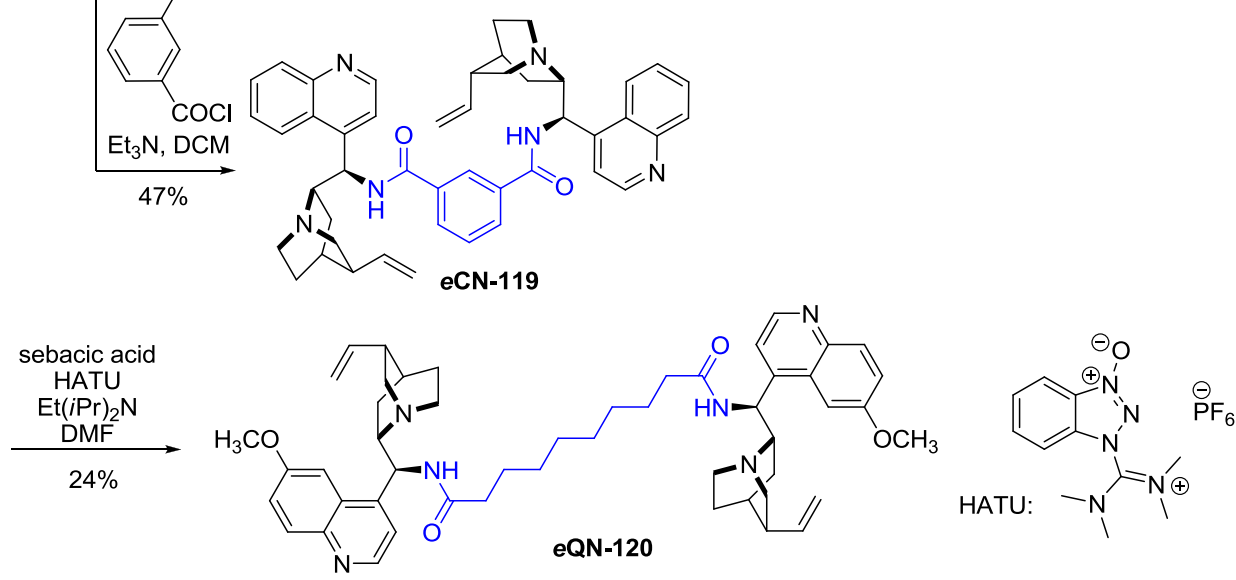

Fig. 31 Synthesis of dimeric amides

Springer 


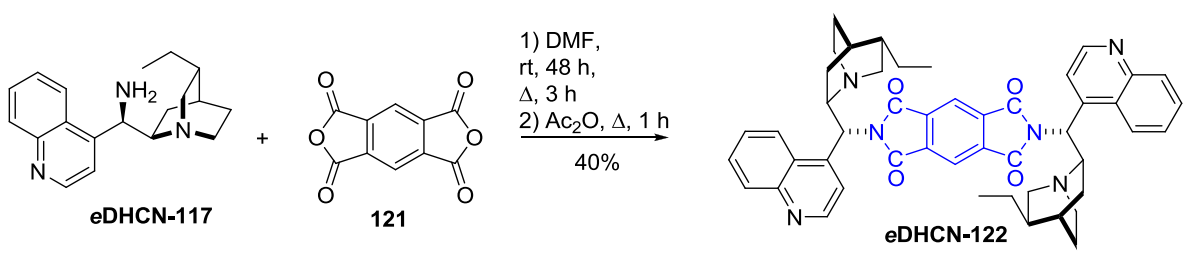<smiles>CC1(C)C2CCNC1C2c1ccnc2ccccc12</smiles>

eDHCN-117<smiles>O=C1OC(=O)c2ccc3c4c(ccc1c24)C(=O)OC3=O</smiles>

123

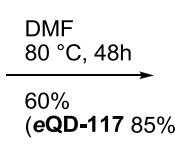

(eQD-117 85\%)

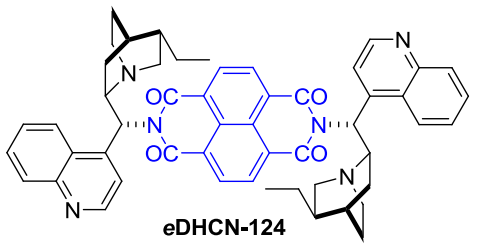
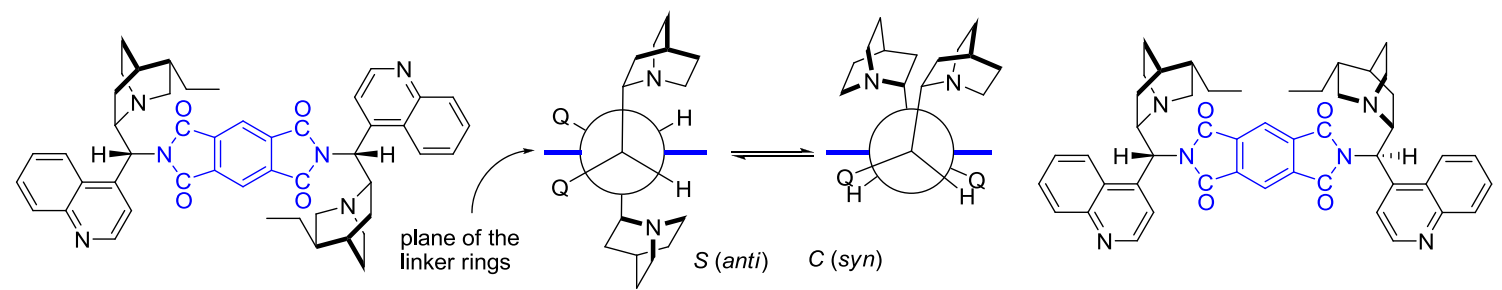

Fig. 32 Synthesis of bisimide and a simplified representation of their conformation equilibrium<smiles>CCC1CC2CCN1CC2C(N)c1ccnc2ccc(OC)cc12</smiles>

eDHQN-117

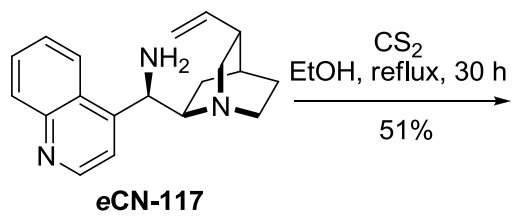

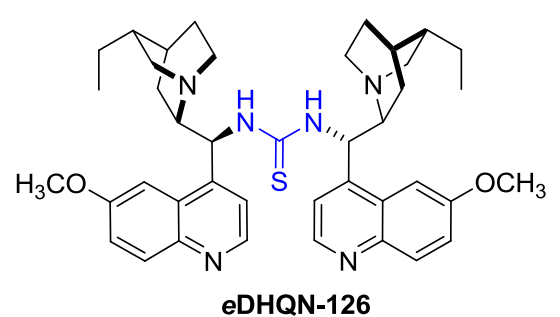

eDHQN-126<smiles>C=C1CC2CC1CN2C(NC(=S)NC(c1ccnc2ccccc12)C1CC2CCC1C2)c1ccnc2ccccc12</smiles>

Fig. 33 Synthesis of dimeric alkaloid thiourea

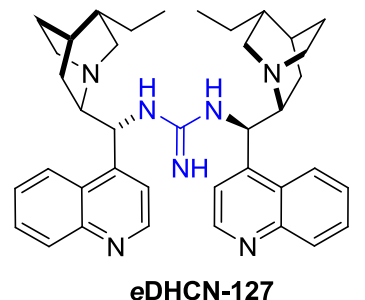

Fig. 34 Guanidine-linked dimer

Thus, dimer $e \mathbf{C N}-\mathbf{1 3 8}$ with two unsymmetrically substituted squaramide units was synthesized, as well as analogous trimers $e \mathbf{C N - 1 3 9}$ and $e \mathbf{C N - 1 4 0 ~ ( F i g . ~ 3 8 ) . ~ F i r s t , ~ 9 - ~}$ aminocinchonine $e \mathbf{C N}-117$ was treated with an equivalent amount of diethyl squarate $(\mathbf{1 3 5} \mathbf{b})$, and the intermediate monoester-monoamide $e \mathbf{C N}-\mathbf{1 3 7}$ was treated with 0.5 equivalents of $m$-xylylenediamine to give a $C 2$ symmetric ana$\log$ of monomeric squaramides. Analogous $C 3$-symmetric trimeric compounds were obtained in $81-87 \%$ yield, when instead of diamine, the reactive $e \mathbf{C N}-\mathbf{1 3 7}$ intermediate was treated with 0.32 equivalents of selected triamines [101]. The trimers $e \mathbf{C N - 1 3 9}$ and $e \mathbf{C N - 1 4 0}$ were effective catalysts in the asymmetric Michael addition of 1,3-dicarbonyl compounds to nitrostyrenes [101] and Friedel-Crafts alkylation reactions [102]. 


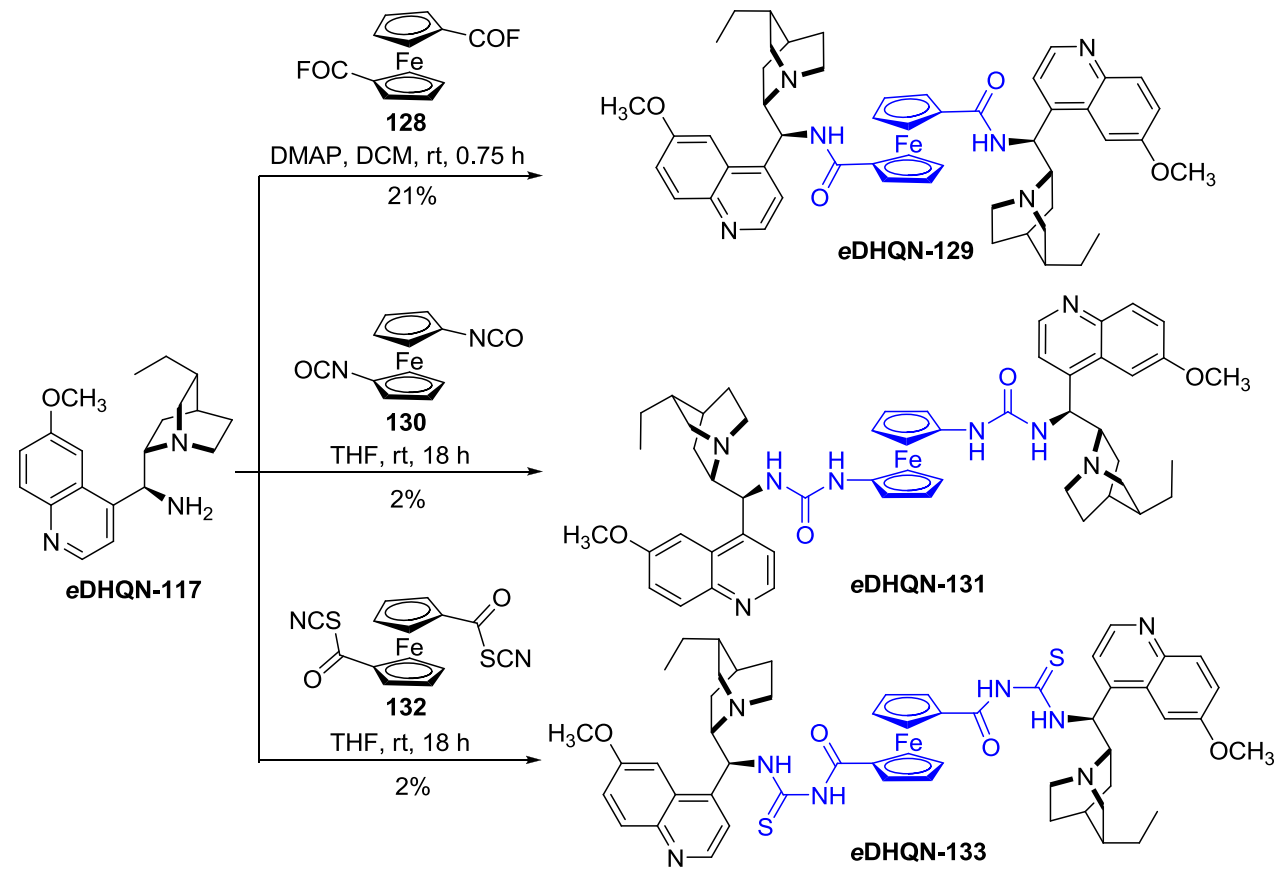

Fig. 35 Synthesis of ferrocene-linked dimers: amide, urea, and thiourea<smiles>CCC1CN2CCC1C2[C@H](NC(=O)c1cc(C(=O)NC(c2ccnc3ccc(OC)cc23)c2ccnc3ccc(OC)cc23)cc(C(=O)N[C@H](c2ccnc3ccc(OC)cc23)C2CC3CCN2C3)c1)c1ccnc2ccc(ON(C)C)cc12</smiles>

Fig. 36 C3-symmetric trimeric Cinchona alkaloid trimer

Although a few monomeric alkaloid sulfonamides are known [103], the only example of the corresponding dimers (with $m$-benzenedisulfonamide linker) appears in the patent literature (Chinese patent No. CN103570708, 2013).

Few Schiff bases obtained from the aminoalkaloids $\mathbf{1 1 7}$ and aromatic aldehydes were described. The reaction of aminoquinine $e \mathbf{Q N}-117$ with phthalic aldehyde gave rise to dimeric imine $e \mathbf{Q N - 1 4 1}$ in $54 \%$ yield. The efficiency of a single step corresponds to the yields achieved with monoaldehydes under similar conditions (75-81\%) [104]. Also, a dendrimeric Schiff base $e \mathbf{C N}-144$ incorporating eight alkaloid units was obtained. The first generation dendrimeric aldehyde $\mathbf{1 4 3}$ was obtained from 5-bromo1,3-bis(dimethoxymethyl)benzene (142) through halogenlithium exchange, reaction with bis(dichlorophosphino) ethane $(75 \%)$, and subsequent hydrolysis of dimethyl acetal (95\%). The condensation of aldehyde 143 with 9-aminocin chonine $e \mathbf{C N}-117$ using trimethyl orthoformate proceeded in nearly quantitative yield (Fig. 39) [105].

\section{9-Carbon- and 9-sulfur-linked dimers}

In our research we demonstrated the formation of dimer $\mathbf{Q N}$ 147 in which the alkaloid units are connected with a direct chemical bond between two C-9 carbon atoms. This product was obtained by treating quinine-derived 9-halides 145-146<smiles>CCC1C2CCN1C(C(N)c1ccnc3ccc(OC)cc13)C2</smiles>

eDHQN-117<smiles>COc1c(OC)c(=O)c1=O</smiles>

$135 a$

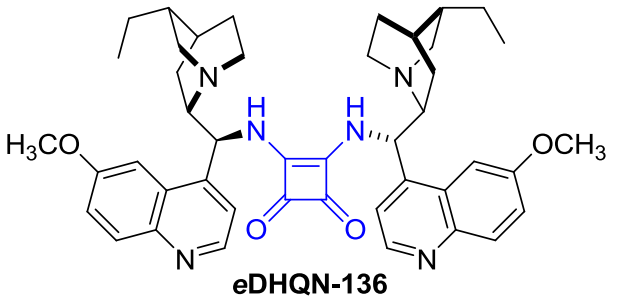

Fig. 37 Representative synthesis of dimeric squaramide 

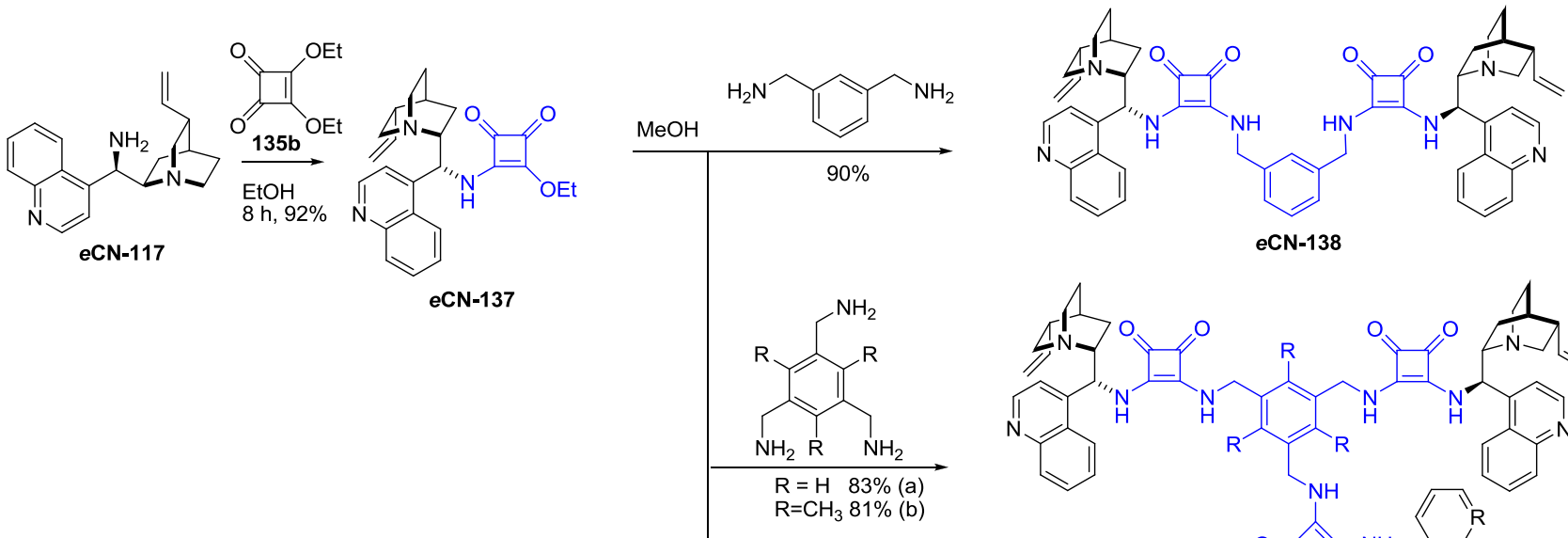

$(137$
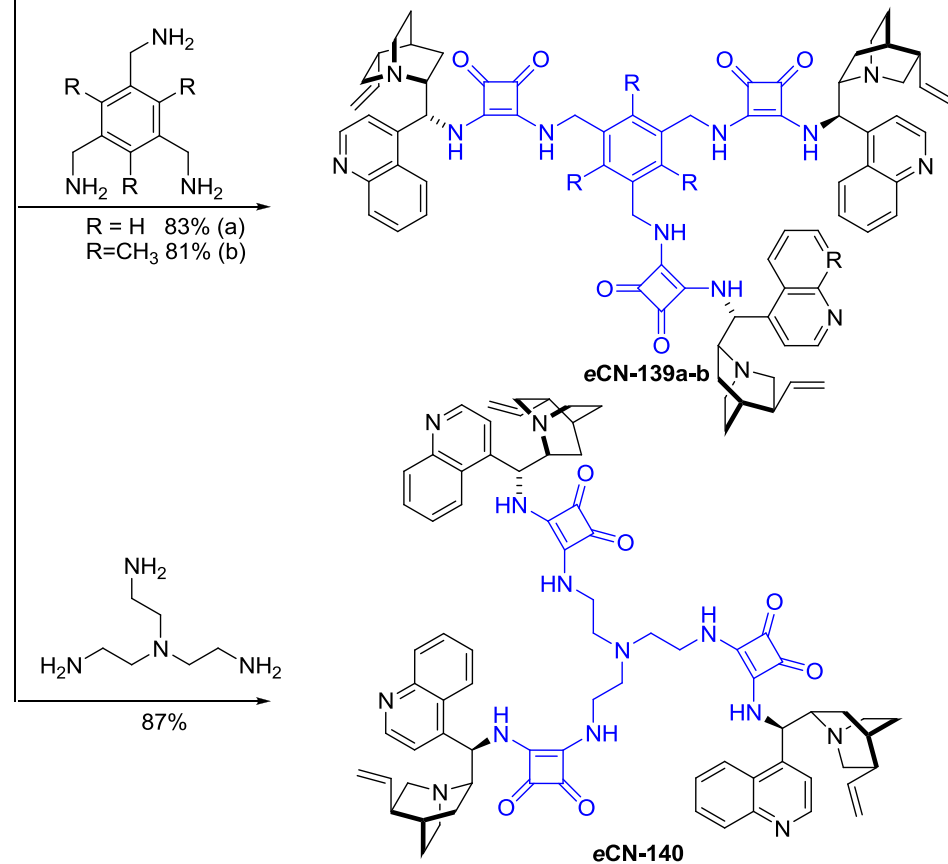

Fig. 38 Synthesis of $C 2$ and $C 3$-symmetric divergently substituted squaramide dimer and trimers<smiles>C=CC1CCN(CC(N)c2cncc3nccc(OC)c23)C1</smiles><smiles>O=Cc1ccccc1C=O</smiles>

QN-117

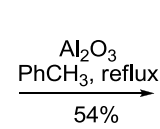

eQN-14<smiles>COC(OC)c1cc([18OH])cc(C(OC)OC)c1</smiles>
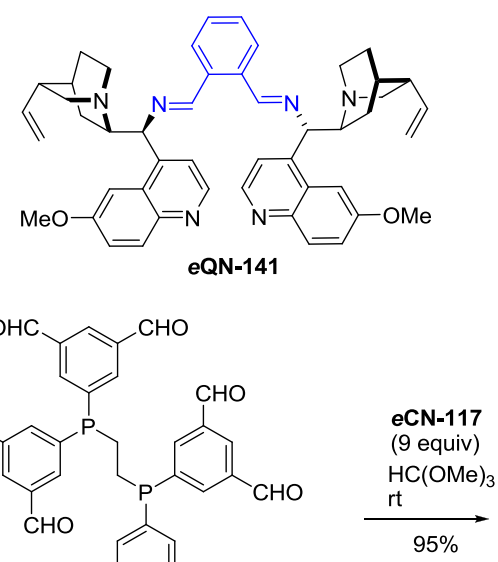

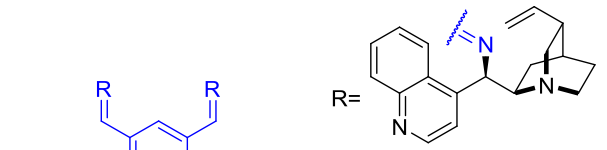

Fig. 39 Synthesis of dimeric and dendrimeric imines

with lithium or butyllithium in THF (Fig. 40). The same isomer of the product $(9 R)$ was obtained from both $9 R$ - and $9 S$ halides: $\mathbf{Q N - 1 4 5}$ and $e \mathbf{Q N - 1 4 5}$, respectively. The most likely explanation for this process was the transient formation of a 9-radical (either direct, or through oxidation of carbanion), followed by radical recombination. The dimers $\mathbf{Q N - 1 4 7}$ and
DHQN-147 are sterically hindered, thus anti and syn atropisomers (arising from rotation of the quinoline ring, Fig. 41) were separated. The rotational barrier was estimated at ca. 23 $\mathrm{kcal} / \mathrm{mol}$ in solution [106]. The reaction of organomagnesium reagents with 9-haloquinine [107] was shown to result in a stereoconvergent Würtz-type coupling (the similarity of this 
<smiles>[X]C(c1ccnc2ccc(OC)cc12)C1CN2CCC1C2C=C</smiles>

QN-145 (9R), $\mathrm{X}=\mathrm{Cl}$

eQN-145 (9S), $\mathrm{X}=\mathrm{Cl}$

eQN-146 (9S), $\mathrm{X}=\mathrm{Br}$

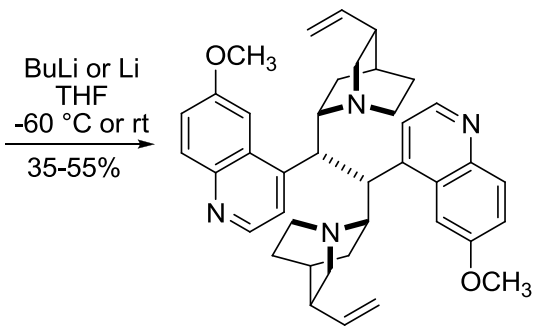

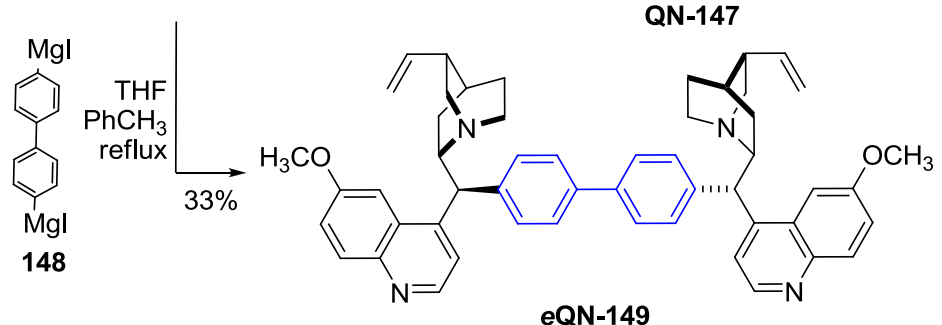

Fig. 40 Dimers bound by C-9 carbon-carbon bonds
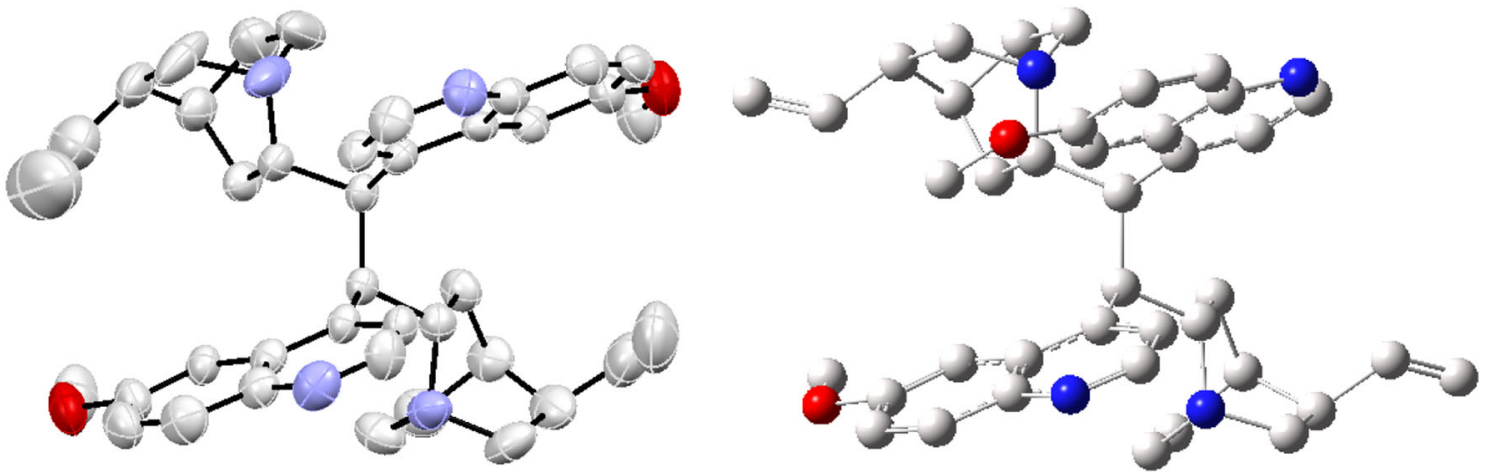

Fig. 41 Atropisomers of the dimer QN-147, X-ray structure (left) and DFT calculated structure for C1 rotamer (right)

process with the direct dimerization to $\mathbf{Q N - 1 4 7}$ is only superficial, since the products had different configuration at C-9) [108]. The extension of this approach with divalent Grignard reagent 148 gave the respective dimer $e \mathbf{Q N}-\mathbf{1 4 9}$ in moderate yield (Fig. 40) [106].

Also, a set of dimeric 9-disulfides was obtained in a sequence of Mitsunobu, reduction, and oxidation reactions. Seven different alkaloids including CD, QN, QD, DHQN, DHQD, as well as 9-epi-quinine ( $e \mathbf{Q N})$, and 9-epi-quinidine $(e \mathbf{Q D})$ reacted giving the corresponding dimers $\mathbf{1 5 2}$ in 24$45 \%$ yield with inversion of configuration. It is noteworthy that the oxidative dimerization step was the least demanding. The authors also regarded dimerization as a means for transient protection of the thiol group in $\mathbf{1 5 1}$, which could be cleanly regenerated with $\mathrm{LiAlH}_{4}$ (Fig. 42) [109].

\section{N1-Qarternary ammonium salts}

Quaternary ammonium salts of Cinchona alkaloids were applied in the 1980's in the asymmetric synthesis under
PTC conditions providing decent level of enantioselection. $C_{2}$-symmetric quaternary ammonium salts derived from binaphthalene emerged as even more effective catalysts in 1999 [110]. Shortly thereafter, highly efficient $C 2$-symmetric dimeric Cinchona alkaloid quaternary ammonium salts were developed [111]. Park and Jew obtained dimers CD-168a, CD-153a, CD-169a in a direct reaction of excess cinchonidine with ortho, meta, and para-xylylene dibromides, respectively, in a mixture of solvents (DMF/EtOH/ $\mathrm{CHCl}_{3}, 6: 5: 2$ $\mathrm{v} / \mathrm{v})$. The obtained dimers were then alkylated at the 9hydroxyl group with allyl bromide. The preparative yields were in the range of 90-94\% over two steps (Fig. 43) [111].

In Park's subsequent development of catalyst CD-153b, a dozen of analogs (CD-156b-167b) were obtained from differently 2- or 5- substituted $m$-xylylene dibromides in 85 $95 \%$ yield [112]. In later works, a quinine analogue was also obtained [113]. The same group synthesized dimers with an extended ring system of the linker, as in naphthalene derivatives DHCD-170-175. The appropriate reactive halides were obtained from dimethylnaphthalenes through radical bromination with NBS ( $88 \%$ yield for 2,7-derivative). Reaction of 


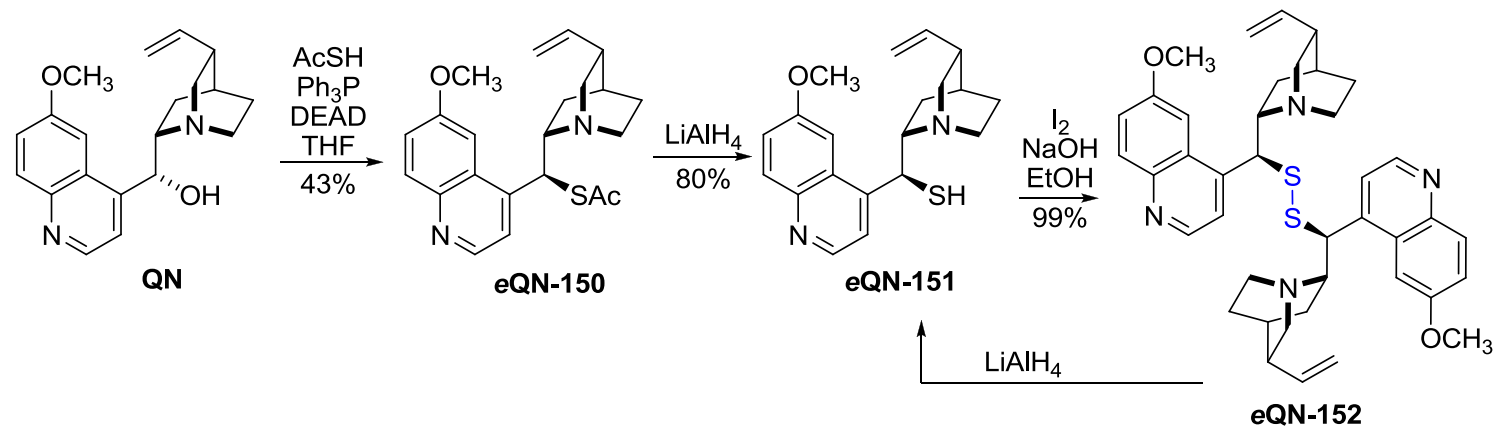

Fig. 42 Representative synthesis of 9-disulfide dimer
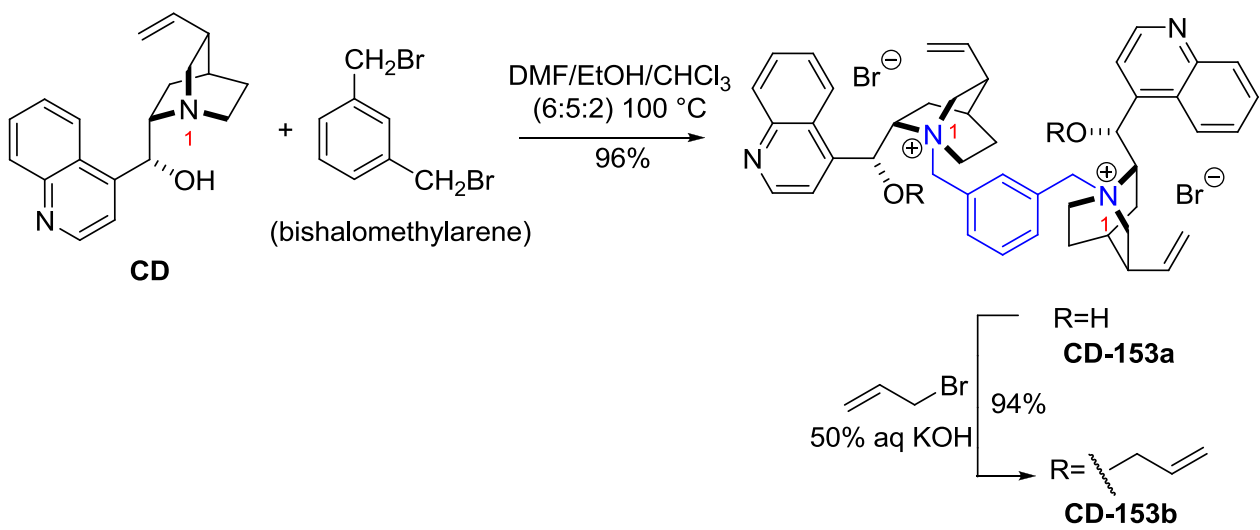

Fig. 43 General synthesis of dimeric ammonium salts

the dibromides with 2.03 equiv of dihydrocinchonidine and dihydrocinchonine and subsequent $9 O$-allylation gave the respective dimers 170b-175b in $90-95 \%$ yield [114]. Further expansion of the ring system was done by the Najera group, who introduced a 9,10-dimethylanthracenyl linker [115]. The required bis(chloromethyl)anthracene (154) was obtained from anthracene, paraformaldehyde, and $\mathrm{HCl}$ [116]. The reaction of $\mathbf{1 5 4}$ with cinchonidine proceeded with slightly better yield than with cinchonine. One of the obtained dimers was subsequently allylated (Fig. 44) [115]. Also dimers CD-177-179 incorporating a 4,4'- [117], 3,3'-, and 2,2'-dimethylbiphenyl linkers as well as DHCD-176 with 3,6-dimethylphenanthrene unit were obtained [39]. Apart from the benzyl-type linkers, also chains of $E$ - and $Z$-butene as well as butyne were applied in the dimers CD-180-182 (Fig. 45) [39].

The dimeric quaternary ammonium salts 153,155 , and 169 were also converted to ionic polymers (e.g., CD-184a) with adequate disulfonates 183a-i. The obtained materials were insoluble in water and most organic solvents (Fig. 46). Nonetheless, these polymers were effective in asymmetric transformations and were easily recovered from the reaction mixture [118,119].

Internal quaternary ammonium salts (i.e., betaines) where both cationic and anionic centers are present within a sin- gle molecule are also known. They differ in acid-base properties with the compounds described previously, in which there is no covalent bond between the oppositely charged species. The Gong group obtained a set of zwitterionic dimers 187 with a binaphthophenolate linker starting from all major Cinchona alkaloids and two axial enantiomers of BINOL [120]. The reactive MOM-protected 3,3'-bis(bromomethyl)BINOL derivative $\mathbf{1 8 5}$ was obtained in 5 steps from commercially available BINOL in $68 \%$ yield [121]. The reaction of enantiomeric $(a R)$-dibromide $\mathbf{1 8 5}$ with the alkaloids afforded the respective dimeric ammonium salts $\mathbf{1 8 6}$ in $64-74 \%$ yields. The coupling was slightly more efficient for the alkaloids of quinine configuration (i.e., $8 S, 9 R$ ). Then, the protecting MOM groups were removed producing betaines 187 in $68-75 \%$ yield (Fig. 47). Diastereomeric dimer $(a S)$-QD-187 was obtained from quinidine and $(a S)$ BINOL derivative $(a S)-\mathbf{1 8 5}$ in overall $\mathbf{7 0} \%$ yield. The authors also obtained species QD-188 with a net-positive charge, using monomethyl BINOL ether (Fig. 48). The obtained dimeric betaines 187-188 were tested as organocatalysts in Mannich reaction of azalactones and aliphatic imines. Dimeric betaine $(a R)-\mathbf{Q D}-\mathbf{1 8 7}$ was particularly successful providing 96-98\%ee [120].

Few dimeric Cinchona alkaloid quaternary ammonium salts incorporate functional linkers relevant to supramolecu- 


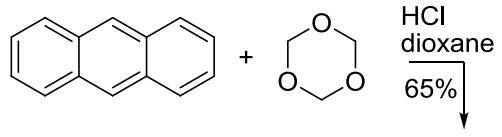<smiles>C=CC1CC2CCN1C2[C@H](O)c1ccnc2ccccc12</smiles><smiles>CC(C)(C)Cc1c2ccccc2c(CCl)c2ccccc12</smiles>
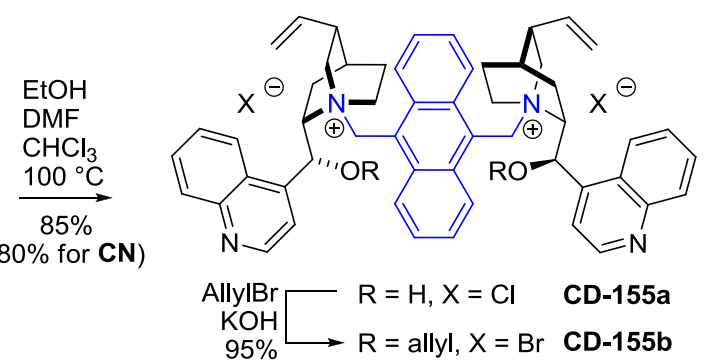

Fig. 44 Synthesis of dimethylanthracene-linked dimer

$\mathrm{R}^{1}$ :<smiles>[R]c1cc(CCC)c([R])c(CCC)c1</smiles>

$\begin{array}{lrll}\underline{R}^{2} & & \underline{R}^{5} & \\ \mathrm{H}, \mathrm{CD}-153 & & \mathrm{H}, \mathrm{CD}-153 \\ \mathrm{~F}, & 156 & \mathrm{~F}, & 162 \\ \mathrm{Cl}, & 157 & \mathrm{Br}, & 163 \\ \mathrm{Br}, & 158 & \mathrm{I}, & 164 \\ \mathrm{NO}_{2}, & 159 & \mathrm{NO}_{2}, & 165 \\ \mathrm{CN}^{2} & 160 & t \mathrm{tBu}, & 166 \\ \mathrm{OCH}_{3}, & 161 & \mathrm{OCH}_{3}, 167\end{array}$<smiles>CCCc1ccc2ccc(CCC)cc2c1</smiles>

DHCD-170<smiles>CCCc1cccc2cccc(CCC)c12</smiles>

DHCD-173<smiles>ICc1ccc2ccc3ccc(CI)cc3c2c1</smiles>

DHCD-176<smiles>ICCc1cccc(-c2cccc(CCI)c2)c1</smiles><smiles>CCC/C=C/CCCC</smiles>

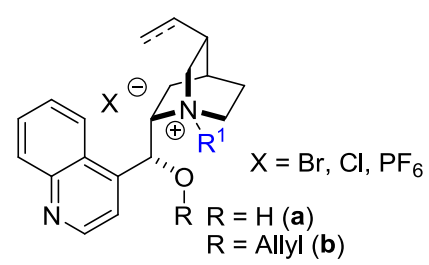<smiles>CCCc1ccccc1CCC</smiles>
CD-168<smiles>CCCc1cc2ccccc2cc1CCC</smiles>
DHCD-171

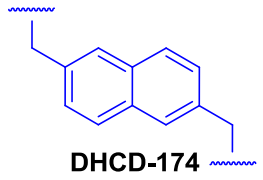

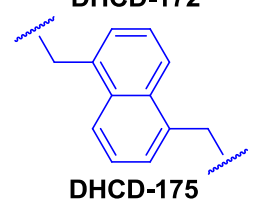<smiles>CCCc1c2ccccc2c(CCC)c2ccccc12</smiles><smiles>CCCc1ccc(-c2ccc(CCC)cc2)cc1</smiles><smiles>CCC/C=C\CCI</smiles><smiles>CCCc1ccccc1-c1ccccc1CCC(C)C(=O)OCc1ccccc1</smiles>

CD-169

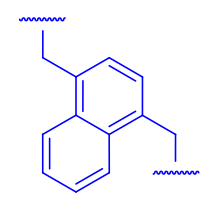

DHCD-172

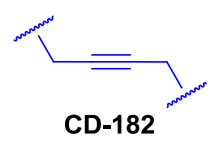

Fig. 45 Linkers in dimeric Cinchona quaternary ammonium salts 


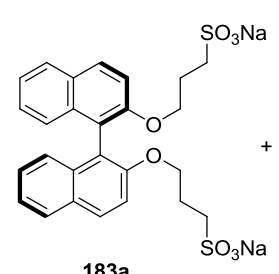

$183 a$

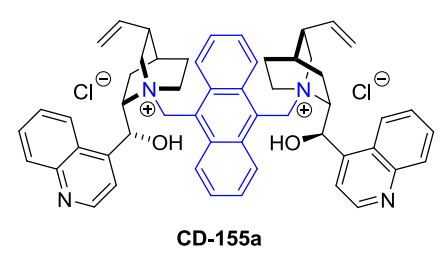

CD-155a

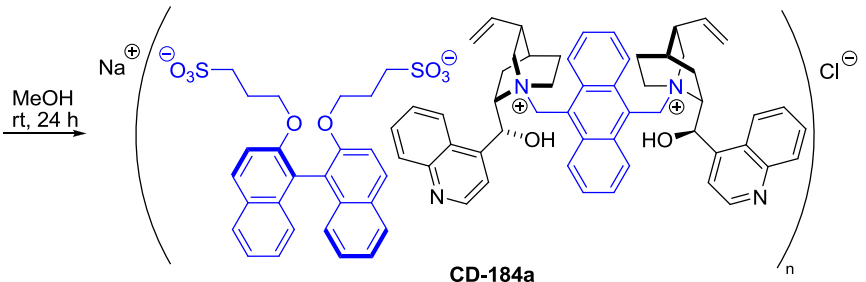

CD-184a

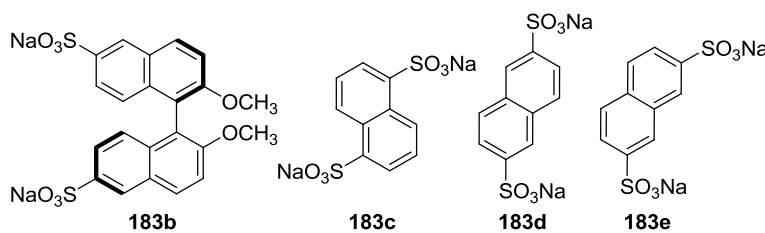
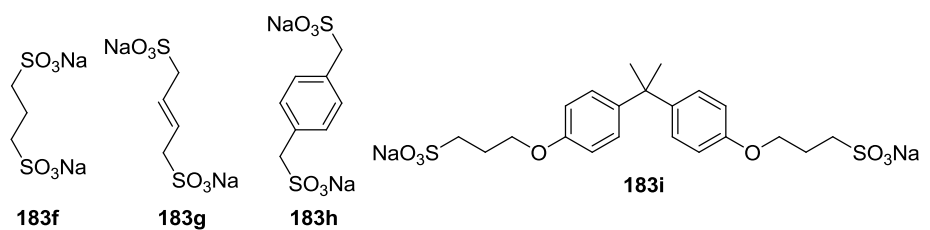

Fig. 46 Ionic polymer of dimeric Cinchona quaternary ammonium salt and structures of disulfonates

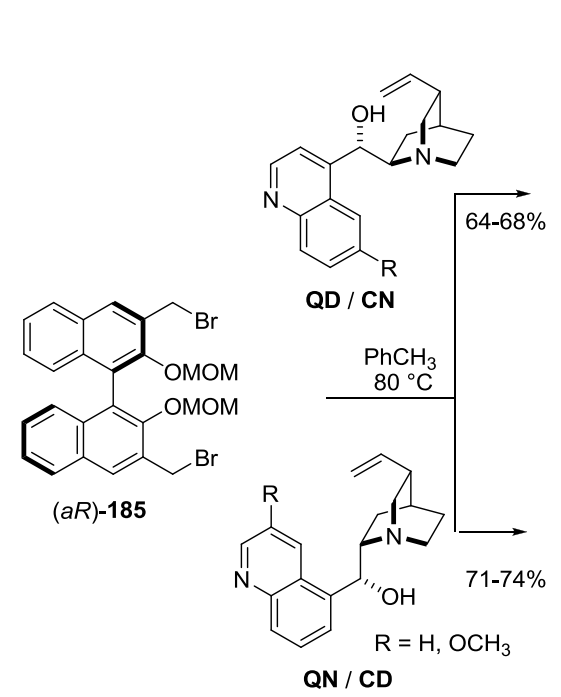

QN / CD

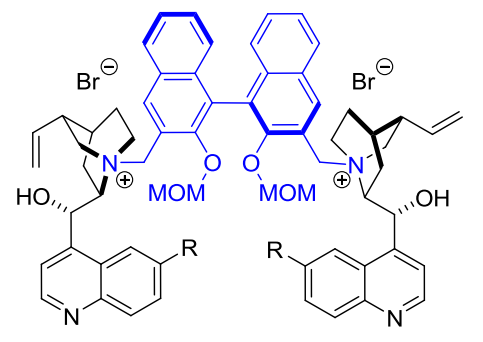

QD-186 / CN-186

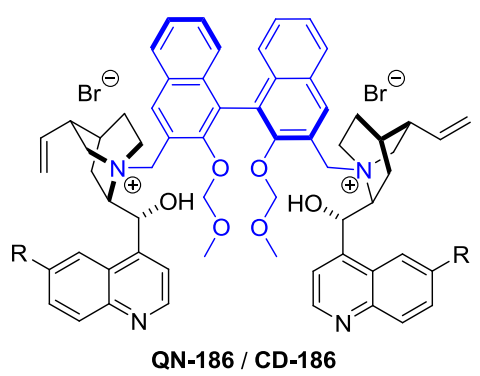

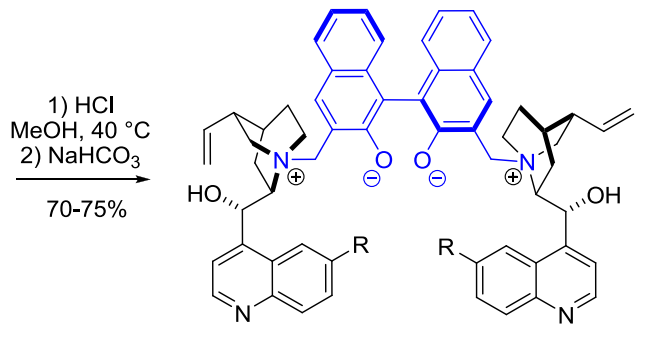

QD-187/ CN-187
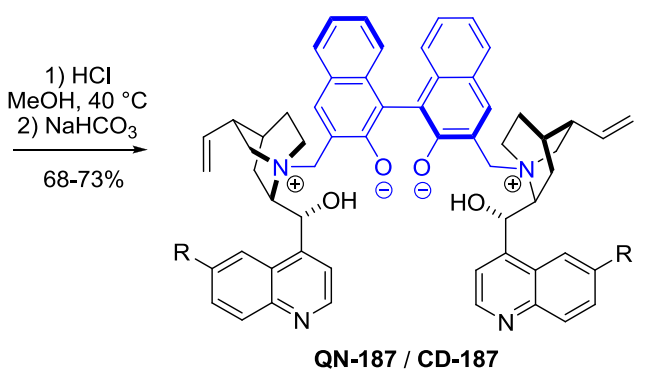

Fig. 47 Synthesis of dimeric betaines

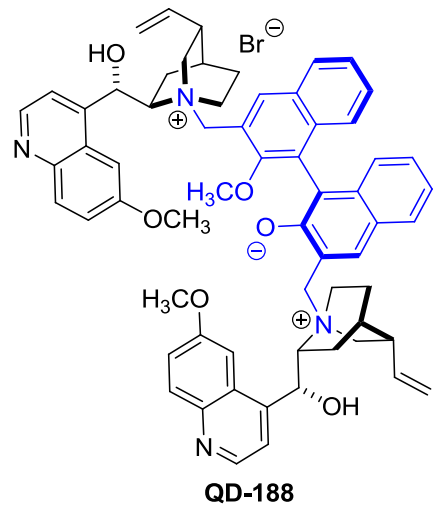

Fig. 48 Example of unsymmetrical dimer

lar chemistry, such as macrocyclic amine 190, calixarene 193, and polyethylene glycol 195. Siva and Murugan obtained di(bromobutyl)tetraazacyclotetradecane derivative, which was subsequently used for double $N 1$-quaternization of both cinchonine $(94 \%)$ and cinchonidine $(87 \%)$. The obtained dimeric products 190a were subsequently $9 O$-allylated to give 190b in high yield (Fig. 49). In the original paper there are, however, some discrepancies between the reported spectral data and structures of 189-190 [122].

Similarly, linkers of various lengths incorporating the calix[4]arene scaffold were synthesized. The reactive dihalides 192a-c were obtained from 4-tert-butylcalix[4] arene (191) and $\alpha, \omega$-dibromoalkanes [123]. The dihalides 192 were then used to $N 1$-alkylate cinchonidine furnishing dimers CD-193a-c. The yield of the coupling increased with the separation between the alkaloid units in $86-96 \%$ range (Fig. 50) [124].

Polyethylene glycol (PEG2000) was also used in the role of a linker [125]. For this purpose, PEG was converted to a reactive intermediate 194 by introduction of terminal 

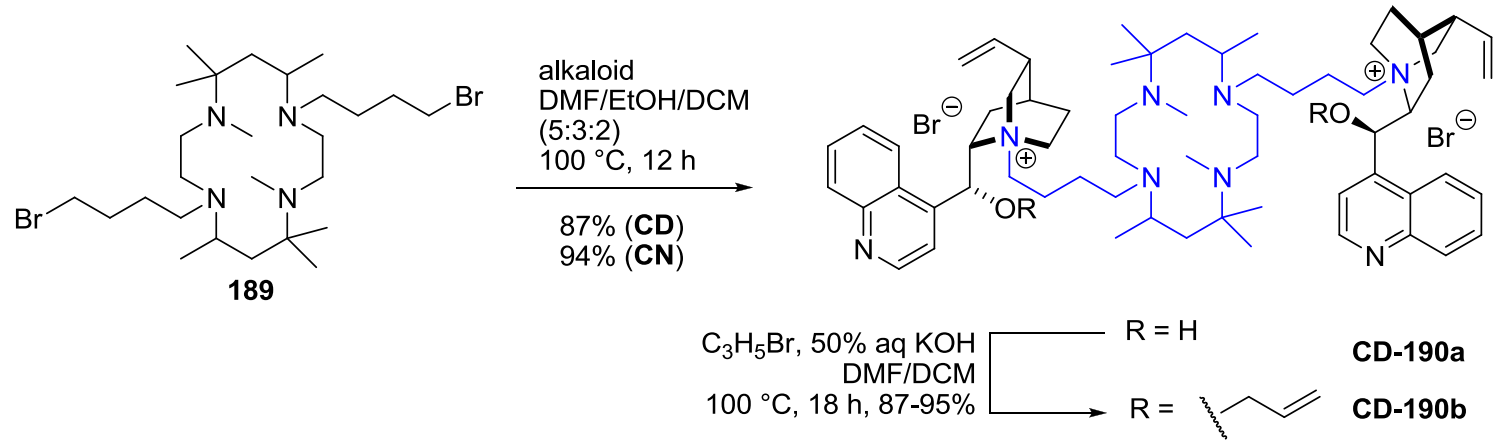

CD-190a

CD-190b

Fig. 49 Synthesis of macrocycle-based dimers
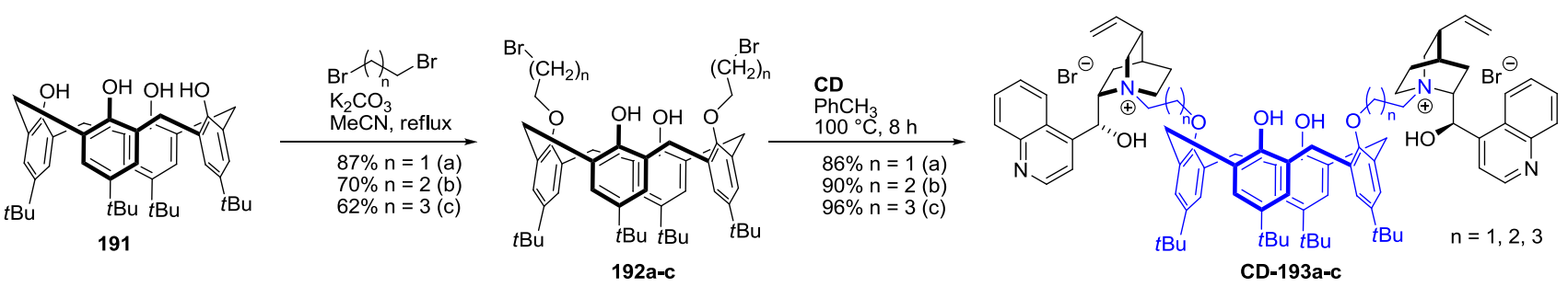

Fig. 50 Synthesis of calix[4]arene-linked dimers
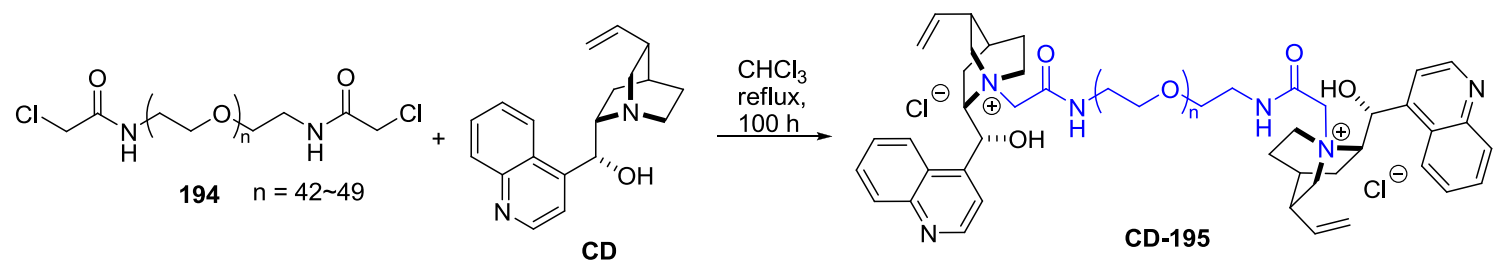

Fig. 51 Synthesis of PEG-linked dimers

chloroacetamide groups [126,127]. A subsequent reaction with the alkaloids ( $\mathbf{C D}, \mathbf{Q N}$, and $\mathbf{C N}$ ) in refluxing chloroform for 4 days afforded the respective dimers 195 (Fig. 51) [125].

It has to be noted that in addition to the dimers, the $C 1$ and $C 3$-symmetric trimers were obtained. The reaction of $\alpha, \alpha^{\prime}, \alpha$ "'-tribromomesitilene (76) and cinchonidine proceeded in nearly quantitative yield and was not impeded by steric interactions. Subsequent $9 O$-allylation gave CD196b in high yield (Fig. 52) [128]. Also, symmetric and nonsymmetric trimers 197-199 with farther separated alkaloid units were obtained in 79-88\% yield (Fig. 53) [129-131].

Quaternary ammonium salts of Cinchona alkaloids were most often employed in asymmetric phase transfer catalysis (PTC), which is useful, for example, for the synthesis of nonracemic amino acids [110,132]. Phenylalanine derivatives can be obtained through enantioselective benzylation of glycine benzophenone imine esters under PTC conditions. This reaction serves as a benchmark for various catalysts (Table 1) [133].

The first generation of Park's catalyst CD-153b, with meta-xylylene linker applied in the PTC benzylation of glycine imine outperformed the monomeric Cinchona cat- alysts both in terms of enantioselectivity and reactivity. On the other hand, application of the isomeric dimer with ortho-xylylene linker gave poor ees. The allylation of the 9-hydroxyl in the catalysts often significantly improved the enantioselectivity in the PTC transformation [111]. Study of analogues of CD-153 revealed that 2-fluorine atom in the xylylene linker as well as 10,11-hydrogenated alkaloid unit further improved the enantioselectivity [112]. A highly hindered trimer CD-196b provided high enantioselectivity (94\% ee at $-20^{\circ} \mathrm{C}$ ) at a cost of reactivity [128] The second generation Park's catalyst DHCD-170b with 2,7-naphthyl link was one of the most efficient and enantioselective catalysts for the benzylation of glycine imine under PTC conditions providing $97 \%$ ee at $0^{\circ} \mathrm{C}$ and $1 \%$ mol loading [114]. This catalyst is commercially available and can be acquired from major reagent suppliers (Fig. 54).

Functional linkers in the dimeric quaternary ammonium salts, in some cases improved their application scope or facilitated the recycling of the catalyst. The macrocyclic dimer CD-190b was claimed to be more suitable for PTC reactions carried in low base concentration [122]. Incorporation of PEG within the linker facilitated recovery of the PTC epoxidation catalyst CD-195 [125]. 


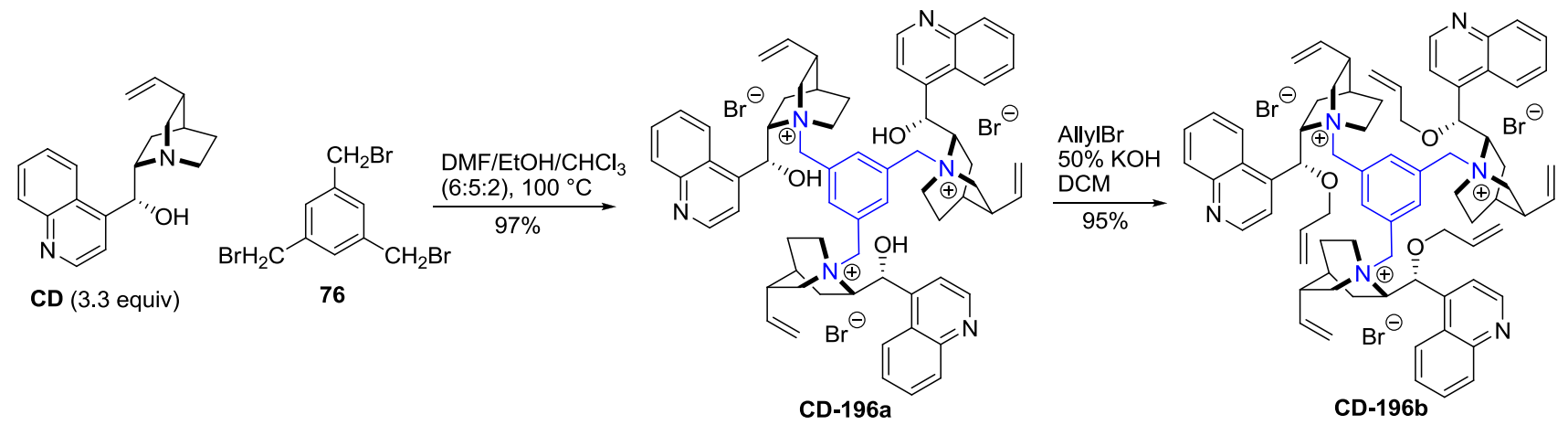

Fig. 52 Synthesis of $C 3$-trimer
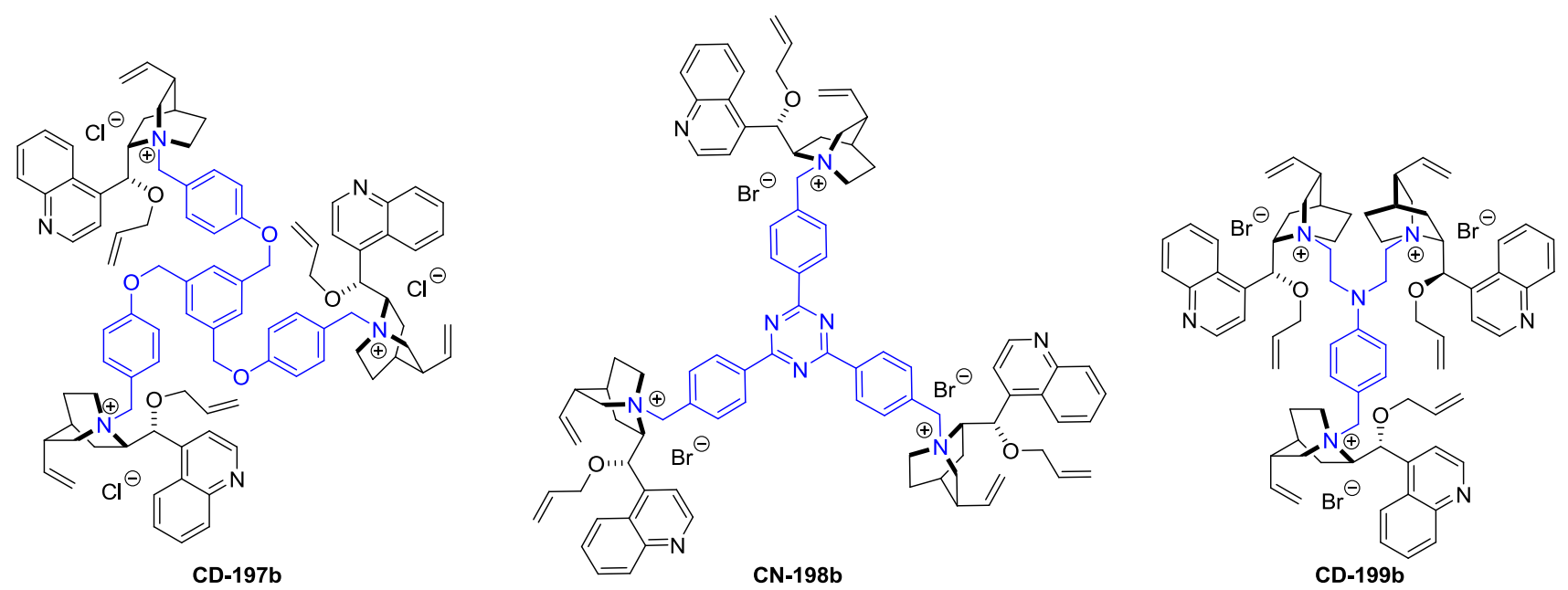

Fig. $53 C 3$ and $C 1$-symmetric trimeric Cinchona alkaloid ammonium salts

Unfortunately, in many of the studied transformations the replacement of cinchonidine with pseudoenantiomeric cinchonine units resulted in more or less noticeably lower enantioselectivity and conversion. Another major concern in asymmetric catalysis is that the success of a particular catalyst structure in one reaction (e.g., PTC alkylation of glycine imine) does not necessarily translate to other asymmetric processes. For example, epoxidation of enones required a catalyst with free 9-hydroxyl group in the alkaloid unit, and the best results were obtained for quinine analogue $\mathbf{Q N}$ 156a. Moreover, this epoxidation failed to proceed enantioselectively when using analogous monomeric catalysts [113]. Cyanation of aldehydes gave good enantioselectivities with CD-155a [134] and so did the Mannich reaction of azalactones catalyzed by QD-187 (up to $99 \%$ ee for adequately modified reactants) (Fig. 55) [187].

\section{3-Vinyl group}

The vinyl group of Cinchona alkaloids is an attractive site of derivatization and was often used to couple the alkaloid (and even their dimers) to solid support. This was often achieved by a 'click' thiol-ene radical addition. The corresponding reaction of dithiols (butane-1,4-dithiol, and 2-mercaptoethyl ether) and cinchonidine led to dimeric products CD-200a$\mathbf{b}$ in fair yields [135]. The dimers were subsequently polymerized by tethering at the quinuclidine nitrogen atom (vide supra) producing an array of polymers CD-201 (Fig. 56). These were assayed in the asymmetric benzylation of glycine imine under PTC conditions providing 71-88\%ee.

Similar polymers, tethered alternatively by quinuclidine nitrogen and vinyl groups, were developed using the Mizoroki-Heck reaction at the alkaloid vinyl groups. First, the dimer CD-202 was obtained in a palladium-catalyzed reaction of cinchonidine and 4,4'-diiodobiphenyl and subsequently was polymerized by quaternization with $4,4^{\prime}$ bis(chloromethyl)biphenyl. Polymers CD-203 were effective in the benzylation of $N$-diphenylmethylene glycine tert-butyl ester. Surprisingly, when using an inverted reaction sequence, i.e., polymerization of the dimeric quaternary ammonium salt CD-179a in a Heck reaction, the obtained polymeric material CD-203 had superior catalytic qualities (Fig. 57) [136]. 
Table 1 Benzylation of $N$-diphenylmethylene glycine tert-butyl ester catalyzed by quaternary ammonium salts

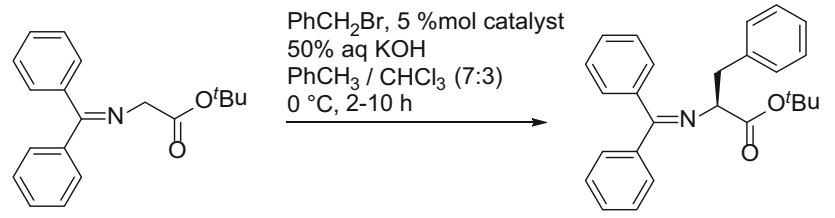

\begin{tabular}{llll}
\hline Catalyst & Time, $\mathrm{h}$ & Yield, \% & Ee, \% (S) \\
\hline DHCD-170b & 0.5 & 95 & 97 \\
DHCD-156b & 6 & 94 & 96 \\
CD-156b & 6 & 93 & 94 \\
DHCD-172b & 2 & 92 & 91 \\
CD-153b & 2 & 91 & 90 \\
CD-155a & 6 & 88 & 86 \\
DHCD-175b & 3 & 90 & 86 \\
CD-179b & & 93 & 84 \\
CD-169b & 4 & 92 & 80 \\
CD-175b & 3 & 92 & 80 \\
DHCD-174b & 3 & 90 & 79 \\
$O$-allyl- $N 1$-benzyl-cinchonidinium & 2 & 92 & 75 \\
bromide & & 84 & 70 \\
CD-155b & 1 & 82 & 44 \\
DHCD-173b & 10 & 88 & 36 \\
DHCD-171b & 10 & 90 & 31 \\
CD-168b & 3 & &
\end{tabular}
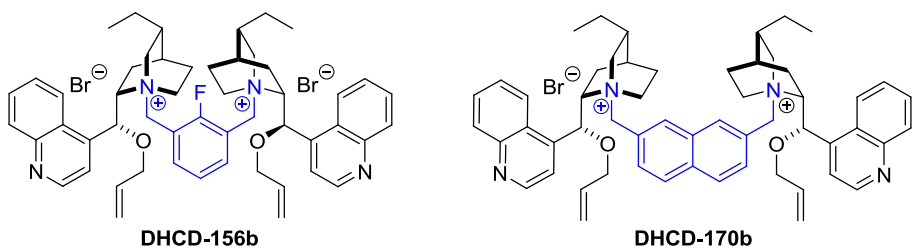

Fig. 54 The most effective 1st and 2nd generation Park's and Jew's catalysts for the alkylation of Schiff bases under PTC conditions

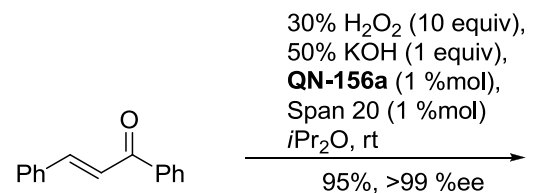

$30 \% \mathrm{H}_{2} \mathrm{O}_{2}$ (10 equiv),

$50 \% \mathrm{KOH}$ (1 equiv),

$95 \%,>99 \%$ ee<smiles>O=C(c1ccccc1)C1OC1c1ccccc1</smiles><smiles>COc1ccc(C=O)cc1</smiles>
$\mathrm{NCCO}_{2} \mathrm{Me}$<smiles>COC(OC)c1ccc(OC(C)C)cc1</smiles><smiles>CC(C)[C@@H]1N=C(c2ccccc2)OC1=O</smiles>
QD-187 (5\%mol)
$\underset{\text { THF, }-40{ }^{\circ} \mathrm{C}, 24 \mathrm{~h}}{78 \%, 43 \% \mathrm{de}, 81} \%$ ee

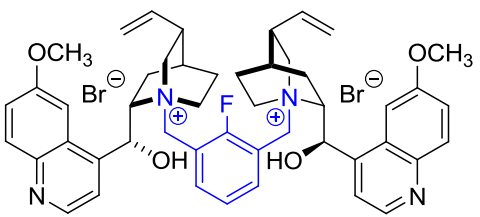

QN-156a catalyst

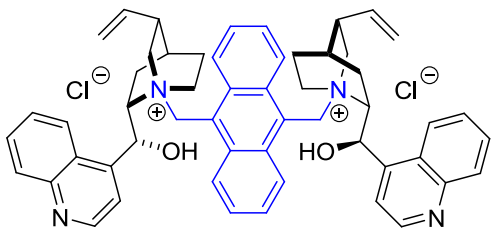

CD-155a catalyst

Fig. 55 Asymmetric reactions catalyzed by dimeric quaternary ammonium salts of Cinchona alkaloids: epoxidation of enones, cyanoformylation of aldehydes, and Mannich reaction 


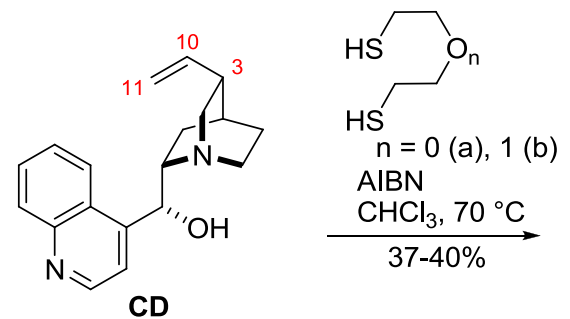<smiles>O[C@H](c1nccc2ccccc12)C1CN2CCC1CC2CCSCCOCCSCCC1CN2CCC1CC2[C@@H](O)[C@H](O)c1ccnc2ccccc12</smiles><smiles>COOC(=O)C(=O)OC</smiles>

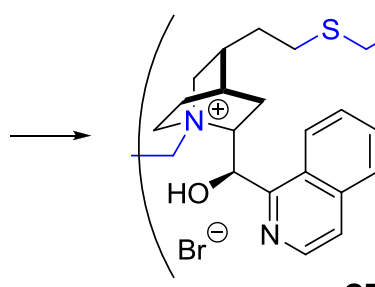<smiles>[R16][R16]([H])([H])[O-]</smiles>

CD-200a-b

Fig. 56 Synthesis of dimer and polymer with dithiol linker

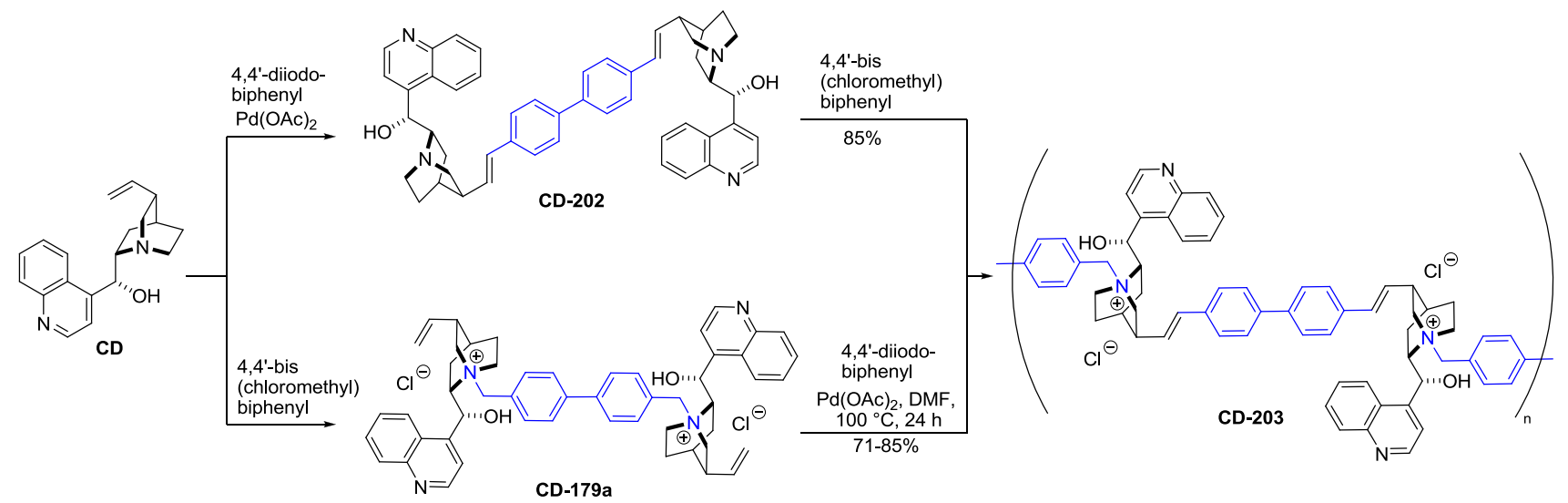

Fig. 57 Synthesis of dimers and polymeric materials using the Mizoroki-Heck reaction
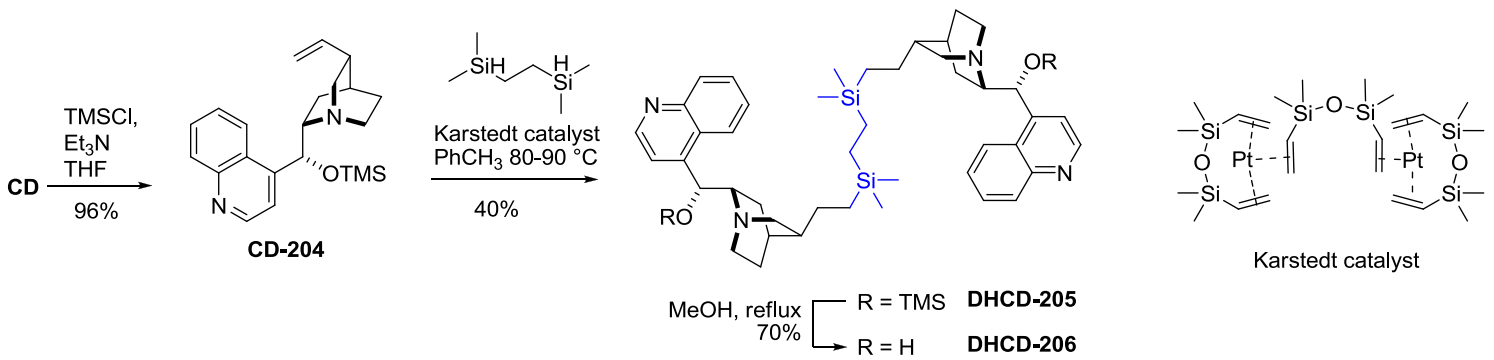

Fig. 58 Synthesis of silicon-linked dimers

Cinchonidine dimer CD-205 tethered at the vinyl groups with silicon linker was also synthesized. First the 9-hydroxyl group of cinchonidine was transiently protected with TMS ether. Then, hydrosilylation of the ether CD-204 with bivalent silane (1,2-bis(dimethylsilyl)ethane) was performed applying Karstedt's catalyst. The coupling gave dimer DHCD-205 in moderate yield, similarly to reactions of CD-204 with other bulky mono-silanes. Finally, the TMS group was removed giving DHCD-206 (Fig. 58). The dimer
CD-206 was applied for modification of $\mathrm{Pt} / \mathrm{Al}_{2} \mathrm{O}_{3}$ catalyst surface for asymmetric hydrogenation of ethyl pyruvate and phenylpropanedione. Unfortunately the enantioselectivity was lower than for unmodified cinchonidine (62 vs. $84 \%$ ee) [137].

The terminal vinyl group is also reactive in alkene metathesis reactions. This approach was used to obtain a series of dimeric phosphite ligands derived from BINOL and Cinchona alkaloids. The phosphite esters QN-208, QD-208, 


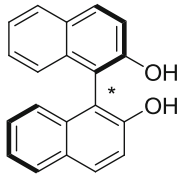

(aR)-207

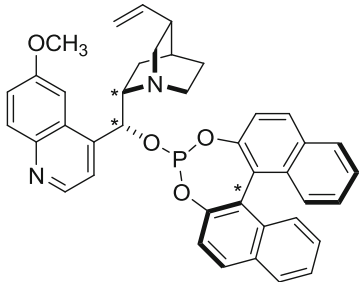

$(a R)-Q N-208$
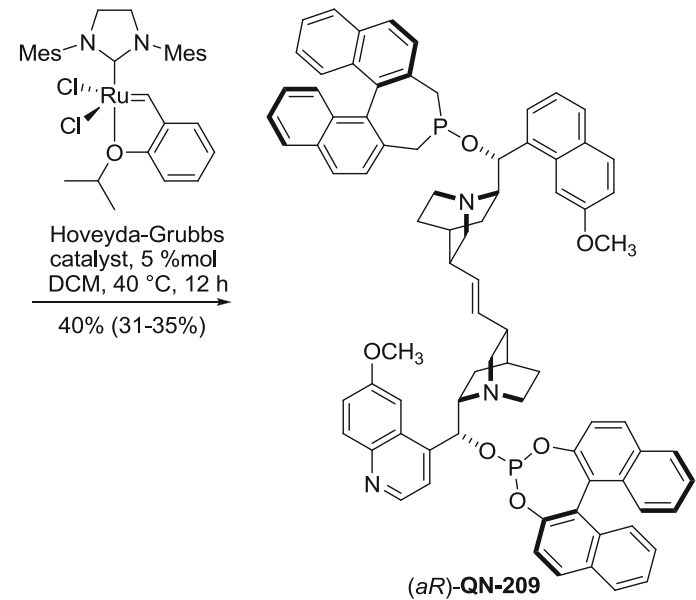

Fig. 59 Metathesis dimerization of Cinchona alkaloid derivatives (for starting materials of different configuration yields are given in parentheses)

and $\mathbf{C N}-208$ were obtained in a one-pot reaction from BINOL (of both $a R$ and $a S$ configurations) and the corresponding alkaloids. The metathesis reaction of $\mathbf{2 0 8}$ with HoveydaGrubbs catalyst produced dimers 209 which were isolated in fair yields (Fig. 59). The authors also obtained libraries containing heterodimers by combining different stereoisomers of the alkaloid phosphites $\mathbf{2 0 8}$ in the metathesis reaction. Dimers 209 along with crude metathesis mixtures were subsequently used in asymmetric iridium-catalyzed asymmetric hydrogenation of $\alpha, \beta$-unsaturated carboxylic acids. Out of the studied compounds, the dimer $(a R)-\mathbf{Q N}-209$ provided up to $92 \%$ ee and outperformed the initial monomer $(\Delta$ ee $8-$ $32 \%$ ) [138].

The vinyl group in the alkaloids can also be converted to a terminal alkyne by bromine addition followed by two $\mathrm{HBr}$ elimination reactions $[139,140]$. The alkynes QN-11 and QD-11 (didehydroalkaloids) were then directly coupled in a Sonogashira-type oxidation reaction. With iodine as an oxidant, the yields were good (71-72\%) and further improved when $9 O$-acetylated didehydroalkaloids $\mathbf{2 1 0}$ were used (86$95 \%$; Fig. 60) [36].

Direct dimerization of $\mathbf{2 1 0}$ using typical Glaser coupling (copper(I) salt $/ \mathrm{O}_{2}$ ) gave merely $15 \%$ yield [36]. In contrast, a similar homocoupling of didehydroalkaloid quaternary ammonium salt QN-212 with copper(II) salt (Elington reaction) proceeded smoothly giving dimer QN-213 in $89 \%$ yield. Also, Sonogashira coupling of the alkaloid alkyne QN-212 with aryl diiodides gave dimers $215 \mathbf{a}-\mathbf{b}$ with phenylene and biphenylene linkers, respectively. Unfortunately, the authors found that the dimerization cannot be performed as a one-pot reaction, instead a stepwise protocol had to be followed using excess of reagents at each coupling step: First QN-212 was coupled with 1,4-diiodobenzene or 4,4' diiodobiphenyl giving iodoaryl derivatives QN-214a and QN-214b, respectively. The subsequent coupling with QN-
212 required significant catalyst loading only to proceed in low yields (Fig. 61). The products QN-213 and QN215a-b were tested in the asymmetric aldol reaction under PTC conditions. Optimum performance was reported for the phenylene-linked dimer QN-215a, although only moderate enantioselectivity was achieved [141].

\section{Quinoline ring}

Quinoline and 6-methoxyquinoline rings also offer useful sites for modification. The $6^{\prime}$-methoxy ether in quinine and quinidine can be cleaved either by $\mathrm{HBr}$ or with alkyl thiolates in DMF giving cupreine and cupreidine, respectively. The latter conditions are tolerant of the 10,11-double bond [142]. The acidity of the phenol group in cupreine (QN-27) and cupreidine (QD-27) facilitates a selective Williamson etherification. The reaction of dihydrocupreine salts with various $\alpha, \omega$-dihaloalkanes provided a library of compounds DHQN-216-218 (Fig. 62). First dimers of this type were reported in the 1920's and used 1,4-E-but-2-ene and butane linkers in DHQN-216 and DHQN-217a, respectively [143]. Cowman obtained a series of dimers with linkers of 4-14 carbon atoms DHQN-217a-f as well as with piperazine-derived linker DHQN-218 [144]. Cupreidine (QD-27) was also dimerized using $m$-xylylene dibromide into QD-219 [66] and trimerized into QD-220 with tris(bromomethyl)benzene [69].

Nitration of the quinoline ring of dihydrocinchonine and dihydrocinchonidine occurs preferentially at the $8^{\prime}$ position. Subsequent reduction of the nitro group with hydrazine on palladium catalyst yielded primary aromatic amine DHCD222. The reactions of $8^{\prime}$-aminoalkaloid with several dicarboxylic acid chlorides yielded the corresponding dimers DHCD-225a-f in good to excellent yields. Similar reactiv- 

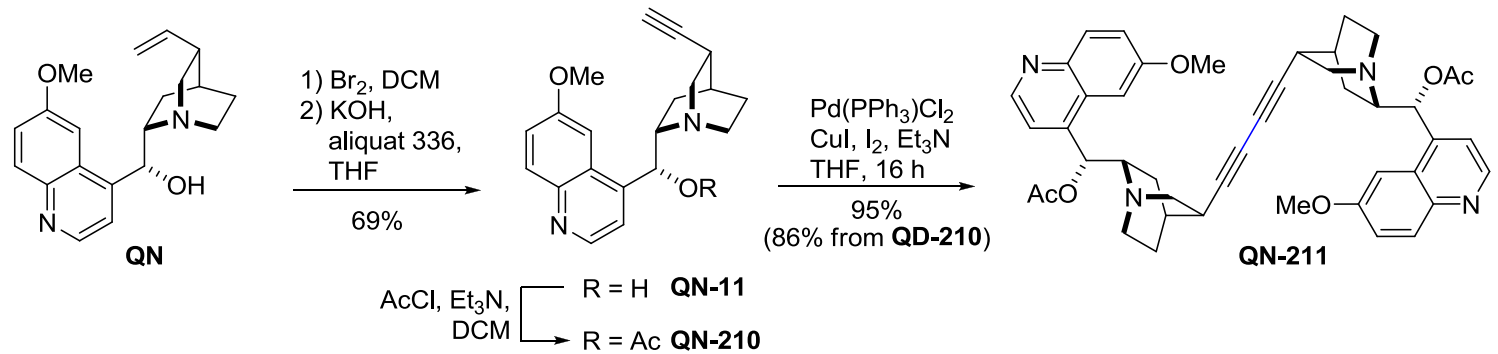

$\mathrm{DCM} \longrightarrow \mathrm{R}=\mathrm{AC}$ QN-210

Fig. 60 Palladium-copper-mediated dimerization of didehydroalkaloids

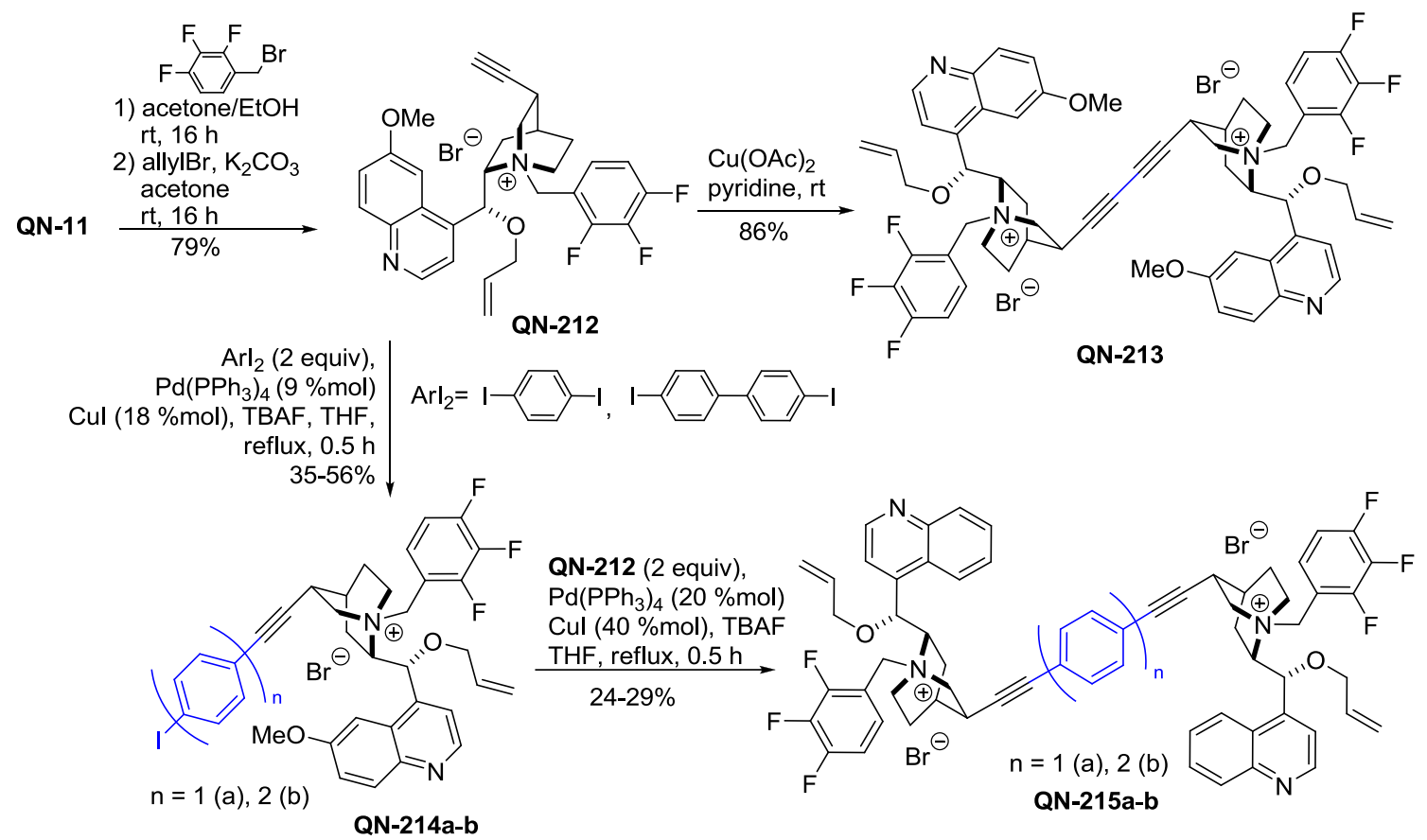

Fig. 61 Dimerization of quaternary ammonium salt

ity of 8'-amino and 9-hydroxy groups required prior use of a transient protecting group (i.e., salicylate) (Fig. 63) [144].

Both 6 -ethers DHQN-217a-f and $8^{\prime}$-amides DHCD225a-f were assayed for inhibition of various strains of Plasmodium falciparum. In vitro tests showed that dimers containing an 8-methylene unit linker were most effective. The amide DHCD-225d $\left(\mathrm{IC}_{50} 0.02-0.05 \mu \mathrm{M}\right)$ was more active than ether DHQN-217c $\left(\mathrm{IC}_{50} 0.08-0.26 \mu \mathrm{M}\right)$. Unfortunately, in vivo study of DHCD-225d revealed lower activity compared to chloroquine and pronounced toxicity [144].

Electrophilic substitution in 6'-metohxy- (QN, QD) and 6'-hydroxyquinoline (QN-27, QD-27) occurs favorably at the $5^{\prime}$-position. Susceptibility of cupreines to such aromatic substitution was exploited in the synthesis of alkaloidderived dyes (Fig. 64). A dimeric dye DHQN-226 was furnished in a reaction of cupreine sodium salt with bisdiazonium salt obtained from benzidine [145]. Also the phenol group in cupreine DHQN-27 was converted to an amine and diazotized. The coupling of the alkaloid $6^{\prime}$ - diazonium salt with cupreine yielded a heterodimer DHQN227 [146].

The 6'-methoxyquinoline ring of Cinchona alkaloids was also partially hydrogenated to give tetrahydroquinoline. The reaction for both $\mathbf{Q N}$ and $\mathbf{Q D}$ led to mixtures of 4 '-epimers which were separated by $2-3$ recrystallizations of mandelic acid salts $(8-15 \%$ yield for pure stereoisomers of 228). The secondary amines $\mathbf{2 2 8}$ were then coupled with glutaconic aldehyde enolate to give polymetine dyes $\mathbf{2 2 9}$ (Fig. 65). The dyes exhibited interesting chiral optical properties: markedly high specific rotation and maximum absorbance at $506-511 \mathrm{~nm}$. The rotatory power was mostly dependent on the configuration at the $4^{\prime}$ center, as exemplified by the $[\alpha]_{\mathrm{D}}(c 0.01, \mathrm{EtOH})$ values of -1545 and +1135 for $\left(4^{\prime} R\right)$ - and $\left(4^{\prime} S\right)$-DHQN-229, respectively [147].

The quinoline ring of the alkaloids is also susceptible to Grignard reagents addition at the $2^{\prime}$ and $4^{\prime}$ positions. In nonetheral solvents the Grignard adds at the $4^{\prime}$-position, and consecutively the deprotonated 9-hydroxyl group adds at the 

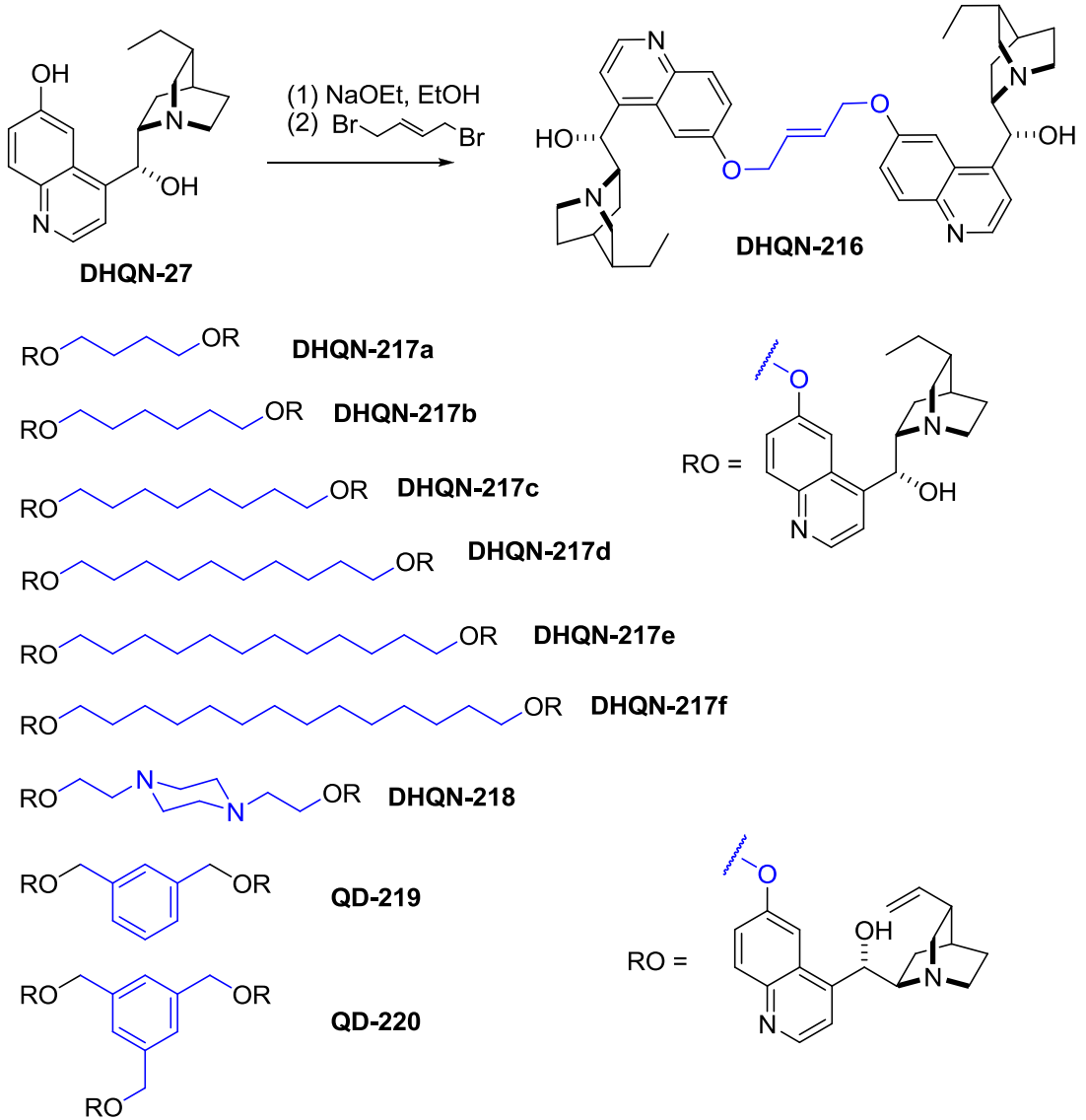

Fig. 62 Dimeric and trimeric cupreine and cupreidine ethers

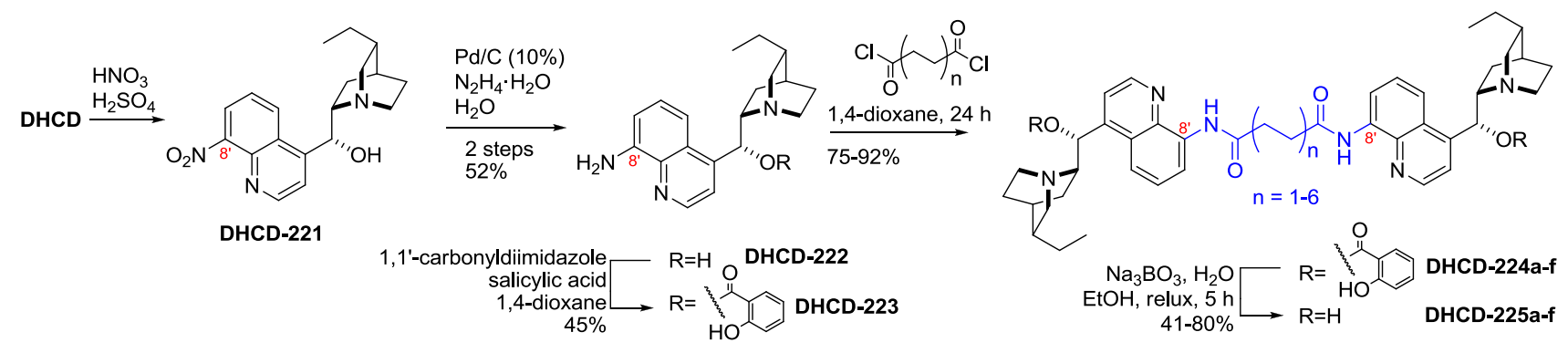

Fig. 63 Synthesis of dimeric $8^{\prime}$-anilides
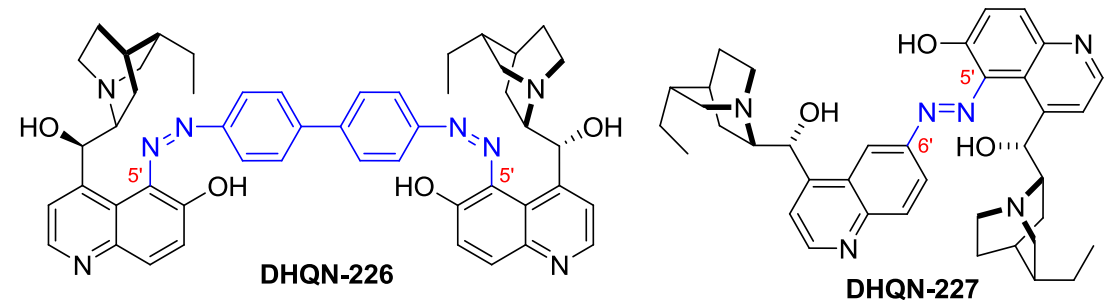

Fig. 64 Dimeric diazo dyes

2 '-position yielding a cyclic aminal with complete diastereoselection. The reaction gave fair yields (typically $65-35 \%$ ) for small and moderately bulky organomagnesium reagents.
Consequently, dimers QN-230 and CD-231 were obtained in 11-12\% yield in a reaction of divalent 1,4-phenylene and 4,4'-biphenylene Grignard reagents, respectively (Fig. 66). 


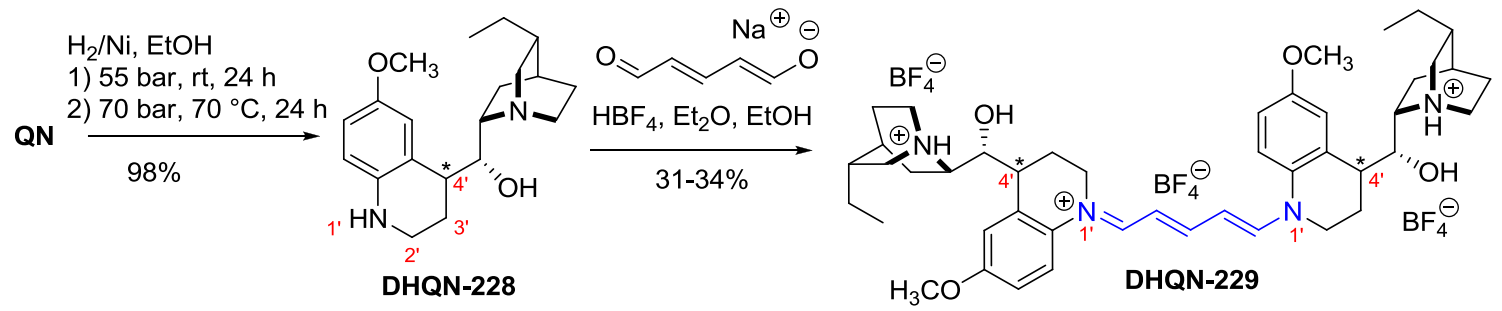

Fig. 65 Synthesis of dimeric polymetine dyes

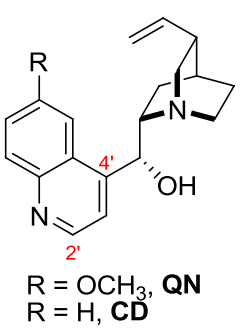

$\mathrm{R}=\mathrm{H}, \mathbf{C D}$

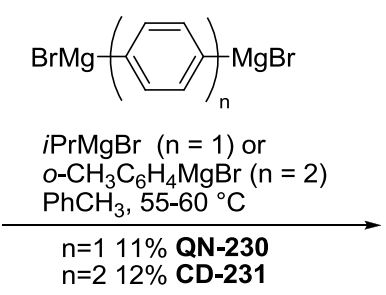

$\mathrm{n}=212 \%$ CD-231

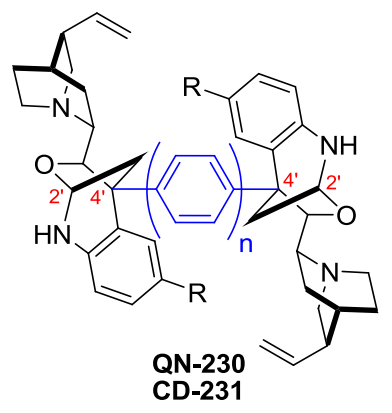

Fig. 66 Formation of $\mathrm{C} 4^{\prime}$-linked dimers

The formation of monomeric products was avoided by using an excess of the less reactive organomagnesium reagents [148].

\section{Double-bridged dimers}

There are only few reported cases where the Cinchona alkaloid units are connected simultaneously at two distinct sites using two independent linkers. These include linear polymers 201, 203 as well as copolymerized compound 10. However, multiple connections between just two alkaloid units would result in a cyclic product of restricted conformation. In studies conducted to explain the stereoselectivity of the asymmetric dihydroxylation, two such structures DHQD-235 and DHQN-237 were described by the groups of Corey and Lohray, respectively. Both used a pyridazine linker attached with 9-ether bond and additionally tethered the dimer by either the vinyl group (235) [149] or the quinoline ring (237) [150]. The 9,11-tethered structure was prepared in a sequence of reactions starting from a Brown hydroboration of quinidine at the vinyl group, followed by protection of the primary 11-hydroxyl group with the triisopropylsilyl (TIPS) group. The protected alcohol DHQD-232 was then coupled using dichloropyridazine (35), and the silyl ether was cleaved to form the dimeric 11-diol DHQD-234. In the last step, esterification of the dimer DHQD-234 with adipoyl chloride gave the bridged dimer DHQD-235, as confirmed by X-ray, in a total of $9.7 \%$ yield over 7 steps (Fig. 67) [149,151].

Lohray obtained cupreine DHQN-27 by cleavage of the $6^{\prime}$-methoxy ether. Then, reaction with 1,5-ditosyloxypentane gave the 6 '-tethered dimer DHQN-236. A subsequent reaction with dichloropyridazine (35) concluded the synthesis of DHQN-237 in 14\% overall yield in 3 steps (Fig. 68) [150].

Asymmetric dihydroxylation reaction using 9,11-double tethered dimer DHQD-235 provided enantioselectivity similar to AD with classic PYDZ-ligand (DHQD-36), and even outperformed it for some olefins. However, the additional 6 '-link in DHQN-237 resulted in much lower level of enantioselectivity (32-72\%ee vs. $88-99 \%$ ee).

Rowan and Sanders studied dynamic self-organization processes of modified Cinchona alkaloids possessing both a reactive ester and hydroxyl functionalities. Instead of careful stepwise tethering of the alkaloid molecules, they built a dynamic library of cyclic oligomeric alkaloids. They hydroborinated the 3-vinyl group, and the resulting 11-alcohol 239 was oxidized to the corresponding acid using the Jones reagent and then esterified to give $\mathbf{2 4 1}$ [152]. In an alternative approach, the 11-alcohol $\mathbf{2 3 9}$ was converted to a halide and etherified with methyl 4-hydroxybenzoate to give $\mathbf{2 4 5}$ [153]. The transestrification of $\mathbf{2 4 1}$ and $\mathbf{2 4 5}$ was carried with catalytic potassium methoxide and 18-crown-6. Under these conditions dynamic libraries of cyclic dimers, trimers, tetramers and acyclic products were formed (Fig. 69). The composition of these mixtures depended on both the linker and the alkaloid configuration. For quinine and cinchonidinederived species, the cyclic trimer was the predominant $(\mathbf{Q N}$ 247b, CD-247b) or nearly the exclusive product (QN-246b, CD-246b) that could be isolated upon crystallization [154]. In the case of quinidine, cyclic dimers were major products both with (QD-247a) and without a $p$-hydroxybenzoic spacer (QD-246a). In mixtures containing multiple types 


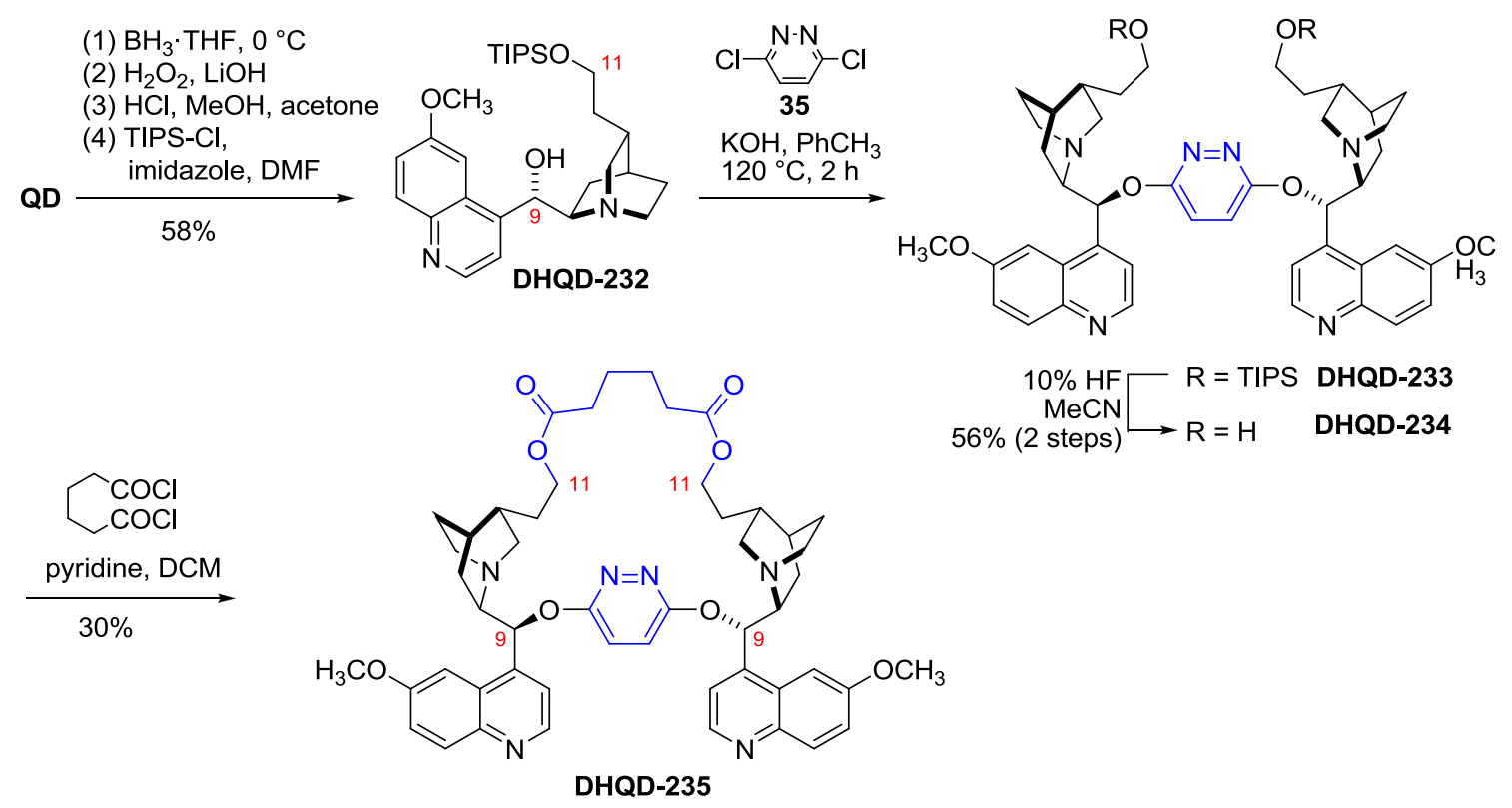

Fig. 67 Synthesis of 9- and 11-tethered dimer

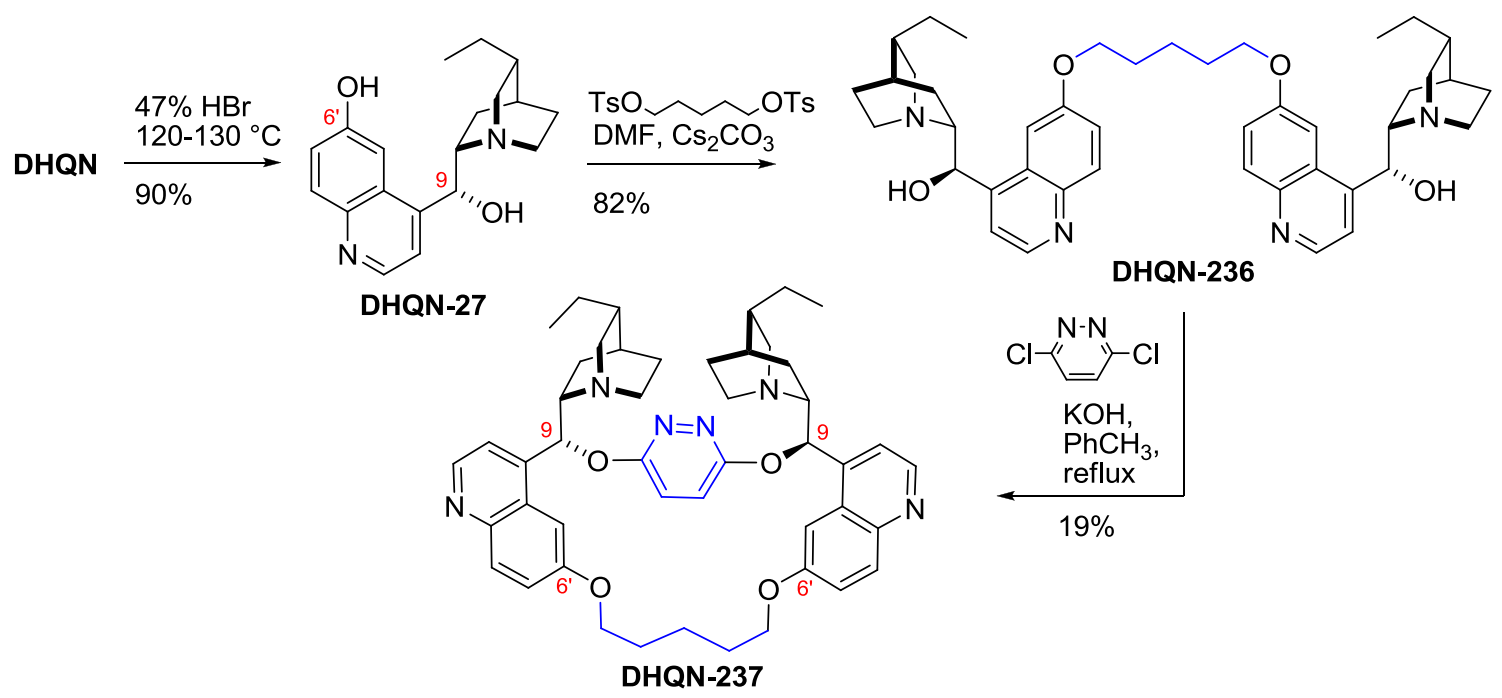

Fig. 68 Synthesis of 9- and 6'-tethered dimer

of precursors, the transesterification resulted in only small amounts of hybrid dimers and oligomers.

Experiments toward the total synthesis of the Cinchona alkaloids led to the formation of an unexpected dimer. One of the steps in Jankowski's synthesis was the aldol condensation of 3-quinuclidinone (248) and quinoline-4-carbaldehyde (249). When this reaction was performed under high pressure conditions, in addition to the desired product $\mathbf{2 5 0}$, a dimeric hemiacetal $\mathbf{2 5 1}$ was formed. The dimer $\mathbf{2 5 1}$ did not dissolve in any of the usual solvents, but heating a suspension in methanol resulted in a cleavage of the hemiacetal bonds to form hydroxyketone $\mathbf{2 5 0}$ (Fig. 70) [155]. The structure of 251 was suggested based on MALDI-MS and IR data, and the relative configuration was not determined.

\section{Closing remarks}

There are many ways of combining multiple Cinchona alkaloid units into dimeric and oligomeric species, few avoid the use of linkers, and even highly hindered dimers can be formed. Most of the dimeric products were a result of planned stepwise syntheses of target molecules; nevertheless, methods of combinatorial chemistry were applied, including formation of dynamic libraries. The most exploited points of chemical diversity involve the central 9 position (Sharplesstype ligands and organocatalysts) and the quinuclidine $\mathrm{N}-1$ nitrogen atom (phase transfer catalysts).

When compared to a monomeric analogue, the dimer has twice the number of reactive polar or electrically charged 


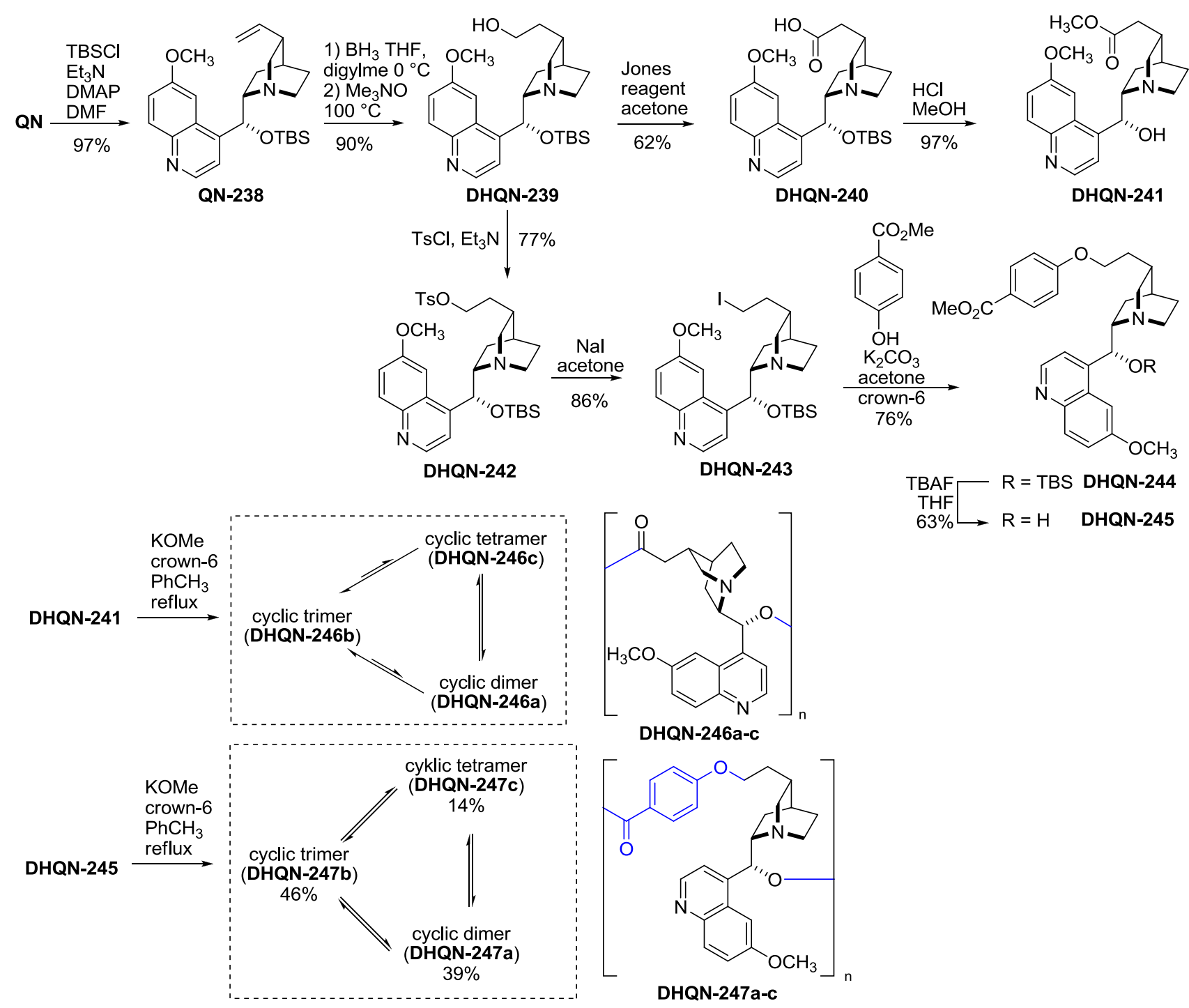

Fig. 69 Synthesis of dynamic self-organizing libraries of cyclic oligomeric Cinchona alkaloids

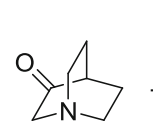

248<smiles>O=Cc1ccnc2ccccc12</smiles>

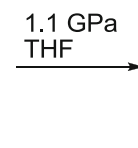<smiles>O=C1CCC2(CCN2C[C@H](O)c2ccnc3ccccc23)C1</smiles>

$250(60 \%)$

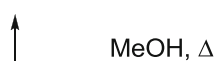

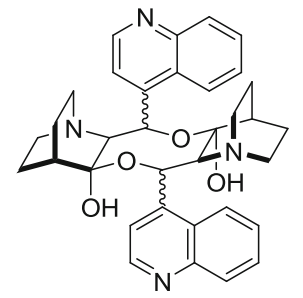

$251(40 \%)$

Fig. 70 High pressure formation of dimeric hemiacetal

groups in close vicinity. The proximity of two bulky units also significantly restricts molecular conformation and may lead to formation of cavities. These factors are of principal significance in catalysis, and, in proper arrangement, have caused certain Cinchona alkaloid dimers to become some of the most effective asymmetric ligands and catalysts. A few of such products are now commercially available (e.g., 3, 51, 61, 170).

The dimeric Cinchona alkaloids are often more basic than their monomeric analogs and were more retained during their 
chromatographical isolation. Apparently, the same phenomenon seems to cause longer retention times on the solid phases modified with dimeric alkaloids.

In medicinal chemistry, the interaction of biomolecules with a tethered dimer may induce functional changes that could contribute to novel pharmacological properties. Few of the studies indicate that biological activity (cytotoxicity, transporter inhibition and antiplasmodial activity) can be found among the Cinchona dimers.

Acknowledgments The author is grateful to the National Science Center NCN, Poland for support (Grant No. 2013/11/D/ST5/02909).

Open Access This article is distributed under the terms of the Creative Commons Attribution License which permits any use, distribution, and reproduction in any medium, provided the original author(s) and the source are credited.

\section{References}

1. Tietze LF, Bell HP, Chandrasekhar S (2003) Natural product hybrids as new leads for drug discovery. Angew Chem Int Ed 42:3996-4028. doi:10.1002/anie.200200553

2. Boyd MR, Hallock YF, Cardellina JH, Manfredi KP, Blunt JW, McMahon JB, Buckheit RW, Bringmann G, Schaeffer M (1994) Anti-HIV Michellamines from Ancistrocladus korupensis. J Med Chem 37:1740-1745. doi:10.1021/jm00038a003

3. Tasler S, Bringmann G (2002) Biarylic biscarbazole alkaloids: occurrence, stereochemistry, synthesis, and bioactivity. Chem Rec 2:113-126. doi:10.1002/tcr.10014

4. Song CE (2009) Cinchona alkaloids in synthesis and catalysis. Wiley, Weinheim

5. Kolb HC, VanNieuwenhze MS, Sharpless KB (1994) Catalytic asymmetric dihydroxylation. Chem Rev 94:2483-2547. doi:10. 1021/cr00032a009

6. Adolfsson H, Zaitsev A (2006) Recent developments in asymmetric dihydroxylations. Synthesis-Stuttgart 2006:1725-1756. doi:10.1055/s-2006-942378

7. Bodkin JA, McLeod MD (2002) The Sharpless asymmetric aminohydroxylation. J Chem Soc Perk T 1:2733-2746. doi:10. 1039/b111276g

8. Donohoe TJ, Callens CKA, Flores A, Lacy AR, Rathi AH (2011) Recent developments in methodology for the direct oxyamination of olefins. Chem Eur J 17:58-76. doi:10.1002/chem.201002323

9. Thakur VV, Sudalai A (2003) $\mathrm{WO}_{3}-30 \% \mathrm{H}_{2} \mathrm{O}_{2}$-cinchona alkaloids: a new heterogeneous catalytic system for the asymmetric oxidation of sulfides and the kinetic resolution of racemic sulfoxides. Tetrahedron Asymmetr 14:407-410. doi:10.1016/ S0957-4166(02)00865-0

10. Kacprzak K, Gawroński J (2001) Cinchona alkaloids and their derivatives: versatile catalysts and ligands in asymmetric synthesis. Synth Stuttg 2001:961-998. doi:10.1055/s-2001-14560

11. Marcelli T, Hiemstra H (2010) Cinchona alkaloids in asymmetric organocatalysis. Synth Stuttg 2010:1229-1279. doi:10.1055/ s-0029-1218699

12. Yoon TP, Jacobsen EN (2003) Privileged chiral catalysts. Science 299:1691-1693. doi:10.1126/science. 1083622

13. Nicolaou KC, Simmons NL, Ying Y, Heretsch PM, Chen JS (2011) Enantioselective dichlorination of allylic alcohols. J Am Chem Soc 133:8134-8137. doi:10.1021/ja202555m
14. Ishimaru T, Shibata N, Horikawa T, Yasuda N, Nakamura S, Toru T, Shiro M (2008) Cinchona alkaloid catalyzed enantioselective fluorination of allyl silanes, silyl enol ethers, and oxindoles. Angew Chem Int Ed 47:4157-4161. doi:10.1002/anie.200800717

15. Lozano O, Blessley G, Thompson AL, Giuffredi GT, Bettati M, Walker M, Borman R, Gouverneur V (2011) Organocatalyzed enantioselective fluorocyclizations. Angew Chem Int Ed 50:8105-8109. doi:10.1002/anie.201103151

16. Chen Y, Tian S-K, Deng L (2000) A highly enantioselective catalytic desymmetrization of cyclic anhydrides with modified Cinchona alkaloids. J Am Chem Soc 122:9542-9543. doi:10.1021/ ja001765+

17. Tang L, Deng L (2002) Dynamic kinetic resolution via dualfunction catalysis of modified Cinchona alkaloids: asymmetric synthesis of $\alpha$-hydroxy carboxylic acids. J Am Chem Soc 124:2870-2871. doi:10.1021/ja0255047

18. Ogawa S, Shibata N, Inagaki J, Nakamura S, Toru T, Shiro M (2007) Cinchona-alkaloid-catalyzed enantioselective direct aldoltype reaction of oxindoles with ethyl trifluoropyruvate. Angew Chem Int Ed 46:8666-8669. doi:10.1002/anie.200703317

19. Poulsen TB, Alemparte C, Saaby S, Bella M (2005) Direct organocatalytic and highly enantio- and diastereoselective mannich reactions of $\alpha$-substituted $\alpha$-cyanoacetates. Angew Chem Int Ed 44:2896-2899. doi:10.1002/anie.200500144

20. McDaid P, Chen Y, Deng L (2002) A highly enantioselective and general conjugate addition of thiols to cyclic enones with an organic catalyst. Angew Chem Int Ed 41:338-340. doi:10.1002/ 1521-3773(20020118)41:2

21. Kowalczyk R, Wierzba AJ, Boratyński PJ, Bąkowicz J (2014) Enantioselective conjugate addition of aliphatic thiols to divergently activated electron poor alkenes and dienes. Tetrahedron 70:5834-5842. doi:10.1016/j.tet.2014.06.035

22. Xue D, Chen Y-C, Wang Q-W, Cun L-F, Zhu J, Deng J-G (2005) Asymmetric direct vinylogous Michael reaction of activated alkenes to nitroolefins catalyzed by modified Cinchona alkaloids. Org Lett 7:5293-5296. doi:10.1021/ol052283b

23. Bella M, Jørgensen KA (2004) Organocatalytic enantioselective conjugate addition to alkynones. J Am Chem Soc 126:5672-5673. doi:10.1021/ja0493594

24. Tian S-K, Deng L (2001) A Highly enantioselective chiral Lewis base-catalyzed asymmetric cyanation of ketones. J Am Chem Soc 123:6195-6196. doi:10.1021/ja010690m

25. Papageorgiou CD, Cubillo de Dios MA (2004) Enantioselective organocatalytic cyclopropanation via ammonium ylides. Angew Chem Int Ed 43:4641-4644. doi:10.1002/anie.200460234

26. Li Y, Liang F, Li Q, Wang Q-R, Jiang L (2011) Room temperature asymmetric allylic trifluoromethylation of MoritaBaylis-Hillman carbonates. Org Lett 13:6082-6085. doi:10. 1021/ol202572u

27. Sharpless KB, Amberg W, Bennani YL, Crispino GA, Hartung J, Jeong KS, Kwong HL, Morikawa K, Wang ZM (1992) The osmium-catalyzed asymmetric dihydroxylation: a new ligand class and a process improvement. J Org Chem 57:2768-2771. doi:10.1021/jo00036a003

28. Norrby P-O, Kolb HC, Sharpless KB (1994) Toward an understanding of the high enantioselectivity in the osmium-catalyzed asymmetric dihydroxylation. 2. A qualitative molecular mechanics approach. J Am Chem Soc 116:8470-8478. doi:10.1021/ ja00098a006

29. Amberg W, Bennani YL, Chadha RK, Crispino GA, Davis WD, Hartung J, Jeong KS, Ogino Y, Shibata T, Sharpless KB (1993) Syntheses and crystal structures of the Cinchona alkaloid derivatives used as ligands in the osmium-catalyzed asymmetric dihydroxylation of olefins. J Org Chem 58:844-849. doi:10.1021/ jo00056a015 
30. Jiang R, Kuang Y, Sun X, Zhang S (2004) An improved catalytic system for recycling $\mathrm{OsO}_{4}$ and chiral ligands in the asymmetric dihydroxylation of olefins. Tetrahedron Asymmetr 15:743-746. doi:10.1016/j.tetasy.2004.01.011

31. Han H, Janda KD (1997) A soluble polymer-bound approach to the sharpless catalytic asymmetric dihydroxylation (AD) reaction: Preparation and application of a [(DHQD $)_{2}$ PHALPEG-OMe] ligand. Tetrahedron Lett 38:1527-1530. doi:10.1016/ s0040-4039(97)00129-9

32. Lee HM, Kim S-W, Hyeon T, Kim BM (2001) Asymmetric dihydroxylation using heterogenized Cinchona alkaloid ligands on mesoporous silica. Tetrahedron Asymmetr 12:1537-1541. doi:10. 1016/S0957-4166(01)00284-1

33. Gavioli E, Maier NM, Minguillón C, Lindner W (2004) Preparative enantiomer separation of dichlorprop with a Cinchona-derived chiral selector employing centrifugal partition chromatography and high-performance liquid chromatography: a comparative study. Anal Chem 76:5837-5848. doi:10.1021/ac040102y

34. Song CE, Yang JW, Ha HJ (1996) Efficient and practical polymeric catalysts for heterogeneous asymmetric dihydroxylation of olefins. Tetrahedron Asymmetr 7:645-648. doi:10.1016/ 0957-4166(96)00054-7

35. Park YS, Yo CH, Choi HY, Kwon EK, Song CE (2004) Osmylated macroporous Cinchona alkaloid resins: highly efficient and recyclable catalysts for asymmetric dihydroxylation of olefins. B Korean Chem Soc 25:1671-1675. doi:10.5012/bkcs.2004.25.11. 1671

36. Seidel MC, Smits R, Stark CB, Frackenpohl J, Gaertzen O, Hoffmann HM (2004) Studies on the asymmetric dihydroxylation of advanced Bryostatin C-ring segments. Synth Stuttg 2004:13911398. doi:10.1055/s-2004-822400

37. Frackenpohl J, Braje WM, Hoffmann HMR (2001) Crosscoupling reactions in Cinchona alkaloid chemistry: arylsubstituted and dimeric quinine, quinidine, as well as quincorine and quincoridine derivatives. J Chem Soc Perk T 1:47-65. doi:10. 1039/b004693k

38. Choi DS, Han SS, Kwueon EK, Choi HY, Hwang SH, Park YS, Song CE (2006) New mono-quarternized bis-Cinchona alkaloid ligands for asymmetric dihydroxylation of olefins in aqueous medium: Unprecedented high enantioselectivity and recyclability. Adv Synth Catal 348:2560-2564. doi:10.1002/adsc.200600253

39. Lee J-H, Yoo M-S, Jung J-H, Jeong B-S (2007) Polymeric chiral phase-transfer catalysts derived from Cinchona alkaloids for enantioselective synthesis of $\alpha$-amino acids. Tetrahedron 63:79067915. doi:10.1016/j.tet.2007.05.076

40. Yang X-W, Liu H-Q, Xu M-H, Lin G-Q (2004) A highly efficient and practical new PEG-bound bi-Cinchona alkaloid ligand for the catalytic asymmetric aminohydroxylation of alkenes. Tetrahedron Asymmetr 15:1915-1918. doi:10.1016/j.tetasy.2004.05.007

41. Becker H, King SB, Taniguchi M, Vanhessche KPM, Sharpless KB (1995) New ligands and improved enantioselectivities for the asymmetric dihydroxylation of olefins. J Org Chem 60:39403941. doi:10.1021/jo00118a005

42. Koermendy K, Ruff F (1990) Pyridazines condensed with a hetero ring: IV Formation of pyrazino [2,3-d]pyridazine skeletons by cyclocondensation of 4,5-diamino- and 3,4,5-triaminopyridazinones. Acta Chim Hung 127:587-599

43. Kolb HC, Bennani YL, Sharpless KB (1993) Short and practical syntheses of (R)-(-)-carnitine and (R)-(-)- $\gamma$-amino- $\beta$ hydroxybutyric acid (GABOB). Tetrahedron Asymmetr 4:133141. doi:10.1016/S0957-4166(00)86023-1

44. Corey EJ, Noe MC, Lin S (1995) A mechanistically designed bisCinchona alkaloid ligand allows position- and enantioselective dihydroxylation of farnesol and other oligoprenyl derivatives at the terminal isopropylidene unit. Tetrahedron Lett 36:8741-8744. doi:10.1016/0040-4039(95)01920-D

45. Hill J, Ehrlich J (1971) Nucleophilic heteroaromatic substitution. II. Phthalazines. J Org Chem 36:3248-3251. doi:10.1021/ jo00820a610

46. Corey EJ, Noe MC, Sarshar S (1993) The origin of high enantioselectivity in the dihydroxylation of olefins using osmium tetraoxide and Cinchona alkaloid catalysts. J Am Chem Soc 115:3828-3829. doi:10.1021/ja00062a080

47. Sun R, Zhang Y, Bi F, Wang Q (2009) Design, synthesis, and bioactivity study of novel benzoylpyridazyl ureas. J Agric Food Chem 57:6356-6361. doi:10.1021/jf900882c

48. Cai L, Cuevas J, Temme S, Herman MM, Dagostin C, Widdowson DA, Innis RB, Pike VW (2007) Synthesis and structureaffinity relationships of new 4-(6-iodo-H-imidazo[1,2-a]pyridin2-yl)-N-dimethylbenzeneamine derivatives as ligands for human $\beta$-amyloid plaques. J Med Chem 50:4746-4758. doi:10.1021/ jm0702231

49. Palamidessi G, Bernardi L (1964) On 2,5-dichloropyrazine1. J Org Chem 29:2491-2492. doi:10.1021/jo01031a536

50. Corey EJ, Noe MC, Ting AY (1996) Improved enantioselective dihydroxylation of bishomoallylic alcohol derivatives using a mechanistically inspired bis-Cinchona alkaloid catalyst. Tetrahedron Lett 37:1735-1738. doi:10.1016/0040-4039(96)00163-3

51. Jumde RP, Mandoli A, De Lorenzi F, Pini D, Salvadori P (2010) Simple preparation of dimeric Cinchona alkaloid derivatives on polystyrene supports and a highly enantioselective catalytic heterogeneous dimerization of ketenes. Adv Synth Catal 352:14341440. doi:10.1002/adsc. 201000165

52. Balzano F, Jumde RP, Mandoli A, Masi S, Pini D, Uccello-Barretta G (2011) Mono- and bis-quinidine organocatalysts in the asymmetric methanolysis of cis-1,2,3,6-tetrahydrophthalic anhydride: A conformational and mechanistic NMR study. Chirality 23:784795. doi:10.1002/chir.20993

53. Crispino GA, Jeong KS, Kolb HC, Wang ZM, Xu D, Sharpless KB (1993) Improved enantioselectivity in asymmetric dihydroxylations of terminal olefins using pyrimidine ligands. J Org Chem 58:3785-3786. doi:10.1021/jo00067a002

54. Calter MA, Wang J (2009) Catalytic, asymmetric Michael reactions of cyclic diketones with $\beta, \gamma$-unsaturated $\alpha$-ketoesters. Org Lett 11:2205-2208. doi:10.1021/o1900586f

55. Calter MA, Phillips RM, Flaschenriem C (2005) Catalytic, asymmetric, "interrupted" Feist-Bénary reactions. J Am Chem Soc 127:14566-14567. doi:10.1021/ja055752d

56. Calter MA, Korotkov A (2011) Catalytic, asymmetric, interrupted Feist-Bénary reactions of $\alpha$-tosyloxyacetophenones. Org Lett 13:6328-6330. doi:10.1021/ol2026697

57. Blagg BSJ, Boger DL (2002) Total synthesis of (+)camptothecin. Tetrahedron 58:6343-6349. doi:10.1016/ S0040-4020(02)00633-6

58. McNamara CA, King F, Bradley M (2004) A triazine core for a new class of Sharpless asymmetric dihydroxylation ligands. Tetrahedron Lett 45:8527-8529. doi:10.1016/j.tetlet.2004.09.098

59. Becker H, Sharpless KB (1996) A new ligand class for the asymmetric dihydroxylation of olefins. Angew Chem Int Ed 35:448451. doi:10.1002/anie.199604481

60. Kim HS, Song Y-M, Choi JS, Yang JW, Han H (2004) Heterogeneous organocatalysis for the asymmetric desymmetrization of meso-cyclic anhydrides using silica gel-supported bis-Cinchona alkaloids. Tetrahedron 60:12051-12057. doi:10.1016/j.tet.2004. 10.046

61. Song Y-M, Seok Choi J, Woon Yang J (2004) Silica gel-supported bis-Cinchona alkaloid: a chiral catalyst for the heterogeneous asymmetric desymmetrization of meso-cyclic anhydrides. Tetrahedron Lett 45:3301-3304. doi:10.1016/j.tetlet.2004.02.079 
62. Bolm C, Maischak A (2001) Asymmetric dihydroxylations using immobilized alkaloids with an anthraquinone core. Synlett 2001:93-95. doi:10.1055/s-2001-9701

63. Wöltinger J, Krimmer H-P, Drauz K (2002) The potential of membrane reactors in the asymmetric opening of meso-anhydrides. Tetrahedron Lett 43:8531-8533. doi:10.1016/ S0040-4039(02)02075-0

64. Wöltinger J, Henniges H, Krimmer H-P, Bommarius AS, Drauz K (2001) Application of the continuous Sharpless dihydroxylation. Tetrahedron Asymmetr 12:2095-2098. doi:10.1016/ S0957-4166(01)00355-X

65. Bui T, Hernández-Torres G, Milite C, Barbas CF (2010) Highly enantioselective organocatalytic $\alpha$-amination reactions of aryl oxindoles: developing designer multifunctional alkaloid catalysts. Org Lett 12:5696-5699. doi:10.1021/ol102493q

66. Bui T, Candeias NR, Barbas CF (2010) Dimeric quinidinecatalyzed enantioselective aminooxygenation of oxindoles: An organocatalytic approach to 3-hydroxyoxindole derivatives. J Am Chem Soc 132:5574-5575. doi:10.1021/ja101032j

67. Lohray BB, Bhushan V, Nandanan E (1996) On the mechanism of asymmetric dihydroxylation of alkenes. Indian J Chem B 35:1119-1122

68. Ahamed P, Haque MA, Ishimoto M, Parvez MM, Haraguchi N, Itsuno S (2013) Synthesis of chiral quaternary ammonium polymers for asymmetric organocatalysis application. Tetrahedron 69:3978-3983. doi:10.1016/j.tet.2013.03.018

69. Siew WE, Ates C, Merschaert A, Livingston AG (2013) Efficient and productive asymmetric Michael addition: development of a highly enantioselective quinidine-based organocatalyst for homogeneous recycling via nanofiltration. Green Chem 15:663. doi:10. 1039/c2gc36407g

70. Suszko J, Szeląg F (1934) O anomalnych własnościach chlorokarbonylowych pochodnych alkaloidów kory chinowej. Rocz Chem 14:1202-1215

71. Lohray BB, Bhushan V (1992) On the mechanism of osmium catalyzed asymmetric dihydroxylation (ADH) of alkenes. Tetrahedron Lett 33:5113-5116. doi:10.1016/S0040-4039(00)61205-4

72. Pires MM, Emmert D, Hrycyna CA, Chmielewski J (2009) Inhibition of P-glycoprotein-mediated paclitaxel resistance by reversibly linked quinine homodimers. Mol Pharmacol 75:92100. doi:10.1124/mol.108.050492

73. Hrycyna CA, Summers RL, Lehane AM, Pires MM, Namanja H, Bohn K, Kuriakose J, Ferdig M, Henrich PP, Fidock DA, Kirk K, Chmielewski J, Martin RE (2014) Quinine dimers are potent inhibitors of the Plasmodium falciparum chloroquine resistance transporter and are active against quinoline-resistant P. falciparum. ACS Chem Biol 9:722-730. doi:10.1021/ cb4008953

74. Hajamis UD, Gadre JN, Pednekar S (1998) Asymmetric dihydroxylation of olefins by osmium tetroxide coordinated with chiral Cinchona alkaloid. Indian J Chem B 37:925-928

75. Chen H, Jin Y, Jiang R, Sun X-L, Li X-Y, Zhang S-Y (2008) New Cinchona alkaloid ester derivatives as catalysts in asymmetric "Interrupted" Feist-Bénary reaction. Catal Commun 9:18581862. doi:10.1016/j.catcom.2008.03.003

76. Palus J, Młochowski J, Juchniewicz L (1998) 2,2'Diselenobisbenzoates and 2,2'-diselenobisbenzenesulfonates: new chiral aryl diselenides. Pol J Chem 78:1931-1936

77. Muñiz K, Nieger M (2003) Ferrocenoyl-substituted Cinchona alkaloids: synthesis, structure, and application in asymmetric catalytic oxidation. Organometallics 22:4616-4619. doi:10.1021/ om0304457

78. Lohray BB, Bhushan V (1993) Kinetic resolution of racemic allylic acetates by asymmetric dihydroxylation (ADH) process: an indication of $\pi-\pi$ interaction. Tetrahedron Lett 34:39113914. doi:10.1016/S0040-4039(00)79261-6
79. Chen H, Wang QF, Sun XL, Luo J, Jiang R (2010) Cinchona alkaloid ester derivatives as ligands in the asymmetric dihydroxylation and aminohydroxylation of alkenes. Mendeleev Commun 20:104-105. doi:10.1016/j.mencom.2010.03.013

80. Suzuki H, Mochizuki K, Hattori T, Takahashi N, Tajima O, Takiguchi T (1988) Asymmetric Diels-Alder reactions of some chiral dienophiles derived from Cinchona alkaloids. B Chem Soc Jpn 61:1999-2005

81. Periasamy M, Ramanathan CR, Kumar NS, Thirumalaikumar M (2001) Asymmetric dihydroxylation of trans-stilbene with a new chiral ligand prepared using dihydrocinchonine and the C2 symmetric chiral trans-9,10-dihydro-9,10-ethanoanthracene11,12-dicarboxylic acid. J Chem Res 2001:512-513. doi:10.3184/ 030823401103168901

82. Kuriakose J, Hrycyna CA, Chmielewski J (2012) Click chemistryderived bivalent quinine inhibitors of P-glycoprotein-mediated cellular efflux. Bioorg Med Chem Lett 22:4410-4412. doi:10. 1016/j.bmcl.2012.04.125

83. Dehmlow EV, Knufinke V (1991) Synthese neuer optisch aktiver Kronenether. Liebigs Ann Chem 1991:1091-1094. doi:10.1002/ jlac. 1991199101188

84. Eilmes J, Ptaszek M, Zielińska K (2001) A new synthetic strategy towards dibenzotetraaza[14]annulenes bearing alkoxycarbonyl and aryloxycarbonyl pendant substituents. Polyhedron 20:143149. doi:10.1016/S0277-5387(00)00600-8

85. Eilmes J, Michalski O, Woźniak K (2001) New chiral receptors based on dibenzotetraaza[14]annulenes. Inorg Chim Acta 317:103-113. doi:10.1016/S0020-1693(01)00342-5

86. Franco P, Lämmerhofer M, Klaus PM, Lindner W (2000) Novel Cinchona alkaloid carbamate C9-dimers as chiral anion-exchange type selectors for high-performance liquid chromatography. J Chromatogr A 869:111-127. doi:10.1016/ S0021-9673(99)00877-8

87. Brunner H, Bügler J, Nuber B (1995) Enantioselective catalysis 98. Preparation of 9-amino(9-deoxy)cinchona alkaloids. Tetrahedron Asymmetr 6:1699-1702. doi:10.1016/ 0957-4166(95)00215-B

88. Brunner H, Bügler J (1997) Enantioselektive Katalyse, 106: 9Amino(9-doxy)Cinchona-Alkaloide und derren Derivate. B Soc Chim Belg 106:77-84

89. Gawroński J, Gawrońska K, Kacprzak K (2001) Chiral C and S conformers of aromatic diimide triads. Chirality 13:322-328. doi:10.1002/chir.1038

90. Kacprzak K, Gawronski J (2003) Bifunctional receptor triad for efficient recognition of mono- and dicarboxylic acids. Chem Commun 13:1532-1533. doi:10.1039/b302003g

91. Kacprzak K, Grajewski J, Gawroński J (2006) Indicator displacement sensor for efficient determination of $\alpha$-hydroxydicarboxylic acids and their chiral discrimination. Tetrahedron Asymmetr 17:1332-1336. doi:10.1016/j.tetasy.2006.03.037

92. Ye J, Dixon DJ, Hynes PS (2005) Enantioselective organocatalytic Michael addition of malonate esters to nitro olefins using bifunctional cinchonine derivatives. Chem Commun 35:44814483. doi:10.1039/b508833j

93. Oh JS, Lee JW, Ryu TH, Lee JH, Song CE (2012) Selfassociation free bifunctional thiourea organocatalysts: synthesis of chiral alpha-amino acids via dynamic kinetic resolution of racemic azlactones. Org Biomol Chem 10:1052-1055. doi:10. 1039/c1ob06629c

94. Károlyi BI, Bősze S, Orbán E, Sohár P, Drahos L, Gál E, Csámpai A (2012) Acylated mono-, bis- and tris- Cinchona-based amines containing ferrocene or organic residues: synthesis, structure and in vitro antitumor activity on selected human cancer cell lines. Molecules 17:2316-2329. doi:10.3390/molecules17032316

95. Vakulya B, Varga S, Csámpai A, Soós T (2005) Highly enantioselective conjugate addition of nitromethane to chalcones using 
bifunctional Cinchona organocatalysts. Org Lett 7:1967-1969. doi:10.1021/o1050431s

96. Jin Z, Xu J, Yang S, Song B-A, Chi YR (2013) Enantioselective sulfonation of enones with sulfonyl imines by cooperative N-heterocyclic-carbene/thiourea/tertiary-amine multicatalysis. Angew Chem Int Ed 52:12354-12358. doi:10.1002/anie. 201305023

97. Ma D, Pan Q, Han F (2002) Diastereoselective Henry reactions of $\mathrm{N}, \mathrm{N}$-dibenzyl $\alpha$-amino aldehydes with nitromethane catalyzed by enantiopure guanidines. Tetrahedron Lett 43:9401-9403. doi:10. 1016/S0040-4039(02)02332-8

98. Malerich JP, Hagihara K, Rawal VH (2008) Chiral squaramide derivatives are excellent hydrogen bond donor catalysts. J Am Chem Soc 130:14416-14417. doi:10.1021/ja805693p

99. Lee JW, Ryu TH, Oh JS, Bae HY, Jang HB, Song CE (2009) Self-association-free dimeric Cinchona alkaloid organocatalysts: unprecedented catalytic activity, enantioselectivity and catalyst recyclability in dynamic kinetic resolution of racemic azlactones. Chem Commun 46:7224-7226

100. Bae HY, Some S, Lee JH, Kim J-Y, Song MJ, Lee S, Zhang YJ, Song CE (2011) Organocatalytic enantioselective Michaeladdition of malonic acid half-thioesters to $\beta$-nitroolefins: From mimicry of polyketide synthases to scalable synthesis of $\gamma$ amino acids. Adv Synth Catal 353:3196-3202. doi:10.1002/adsc. 201100458

101. Min C, Han X, Liao Z, Wu X, Zhou H-B, Dong C (2011) C3Symmetrical cinchonine-squaramide as new highly efficient, and recyclable organocatalyst for enantioselective Michael addition. Adv Synth Catal 353:2715-2720. doi:10.1002/adsc.201100066

102. Han X, Liu B, Zhou H-B, Dong C (2012) Enhanced efficiency of recyclable $\mathrm{C} 3$-symmetric cinchonine-squaramides in the asymmetric Friedel-Crafts reaction of indoles with alkyl trifluoropyruvate. Tetrahedron Asymmetr 23:1332-1337. doi:10.1016/j.tetasy. 2012.08.015

103. Brunner H, Baur Markus A (2003) $\alpha$-Amino acid derivatives by enantioselective decarboxylation. Eur J Org Chem 2003:28542862. doi:10.1002/ejoc.200300206

104. Wei Y, Yao L, Zhang B, He W, Zhang S (2011) Novel Schiff base ligands derived from Cinchona alkaloids for $\mathrm{Cu}(\mathrm{II})$-catalyzed asymmetric Henry reaction. Tetrahedron 67:8552-8558. doi:10. 1016/j.tet.2011.08.076

105. Brunner H, Janura M, Stefaniak S (1998) Enantioselective catalysis: 123: Octaaldehyde type chelating ligands - A divergent synthesis approach to easily tunable expanded ligands for enantioselective catalysis. Synth Stuttg 1998:1742-1749. doi:10.1055/ s-1998-2226

106. Boratyński PJ, Turowska-Tyrk I, Skarżewski J (2008) Stereoselective $\mathrm{C} 9$ carbon-carbon couplings of quinine: synthesis and conformational analysis of new C2-symmetric dimers. J Org Chem 73:7357-7360. doi:10.1021/jo801205n

107. Ochiai E, Tsunashima K, Kobayashi Y (1949) Syntheses of quinine base derivatives. XVI. Synthesis of 9-phenylsubstituent of quinine. Yakugaku Zasshi 69:164-165

108. Boratyński PJ, Turowska-Tyrk I, Skarżewski J (2008) Stereoselective C9 arylation and vinylation of Cinchona alkaloids. Org Lett 10:385-388. doi:10.1021/o17026625

109. Zielińska-Błajet M, Skarżewski J (2009) New chiral thiols and C2-symmetrical disulfides of Cinchona alkaloids: ligands for the asymmetric Henry reaction catalyzed by CuII complexes. Tetrahedron Asymmetr 20:1992-1998. doi:10.1016/j.tetasy.2009.07. 020

110. Hashimoto T, Maruoka K (2007) Recent development and application of chiral phase-transfer catalysts. Chem Rev 107:56565682. doi:10.1021/cr068368n

111. Jew S-S, Jeong B-S, Yoo M-S, Huh H, Park H-G (2001) Synthesis and application of dimeric alkaloid phase-transfer catalysts: $\alpha, \alpha^{\prime}$-bis[(9)-allylcinchonidinium]-, or -xylene dibromide. Chem Commun :1244-1245. doi:10.1039/B102584H

112. Park H-G, Jeong B-S, Yoo M-S, Lee J-H, Kim MG (2003) Highly efficient ortho-fluoro-dimeric Cinchona-derived phasetransfer catalysts. Tetrahedron Lett 44:3497-3500. doi:10.1016/ S0040-4039(03)00687-7

113. Jew S-S, Lee J-H, Jeong B-S, Yoo M-S, Kim M-J, Lee Y-J, Lee J, Lee K, Lah MS (2005) Highly enantioselective epoxidation of 2,4diarylenones by using dimeric Cinchona phase-transfer catalysts: enhancement of enantioselectivity by surfactants. Angew Chem Int Ed 44:1383-1385. doi:10.1002/anie.200462254

114. Park H-G, Jeong B-S, Yoo M-S, Lee J-H, Park M-K, Lee Y-J, Kim M-J, Jew S-S (2002) Highly enantioselective and practical Cinchona-derived phase-transfer catalysts for the synthesis of $\alpha$ amino acids. Angew Chem Int Ed 41:3036-3038. doi:10.1002/ 1521-3773(20020816)41:16

115. Chinchilla R, Mazón P, Nájera C (2002) New dimeric anthracenyl-derived Cinchona quaternary ammonium salts as phase-transfer catalysts for the asymmetric synthesis of $\alpha$ amino acids. Tetrahedron Asymmetr 13:927-931. doi:10.1016/ S0957-4166(02)00211-2

116. Miller MW, Amidon RW, Tawney PO (1955) Some mesosubstituted anthracenes. I. 9,10-Bis-(chloromethyl)-anthracene as a synthetic intermediate. J Am Chem Soc 77:2845-2848. doi:10. 1021/ja01615a053

117. Li L, Zhang Z, Zhu X, Popa A, Wang S (2005) Asymmetric alkylation of a tert-butyl benzophenone Schiff base derivative in water. Synlett 2005:1873-1876. doi:10.1055/s-2005-871559

118. Parvez MM, Haraguchi N, Itsuno S (2012) Molecular design of chiral quaternary ammonium polymers for asymmetric catalysis applications. Org Biomol Chem 10:2870-2877. doi:10.1039/ C2OB06909A

119. Parvez MM, Salam MA, Haraguchi N, Itsuno S (2012) Synthesis of chiral ionic polymers containing quaternary ammonium sulfonate structure and their catalytic activity in asymmetric alkylation. J Chin Chem Soc 59:815-821. doi:10.1002/jccs. 201100724

120. Zhang W-Q, Cheng L-F, Yu J, Gong L-Z (2012) A chiral bis(betaine) catalyst for the Mannich reaction of azlactones and aliphatic imines. Angew Chem Int Ed 51:4085-4088. doi:10. 1002/anie.201107741

121. Li H, Da C-S, Xiao Y-H, Li X, Su Y-N (2008) Direct asymmetric aldol reaction of aryl ketones with aryl aldehydes catalyzed by chiral BINOL-derived zincate catalyst. J Org Chem 73:7398-7401. doi:10.1021/jo801182n

122. Siva A, Murugan E (2005) Syntheses of new dimeric-Cinchona alkaloid as a chiral phase transfer catalysts for the alkylation of Schiff base. J Mol Catal A Chem 241:111-117. doi:10.1016/j. molcata.2005.06.059

123. Li Z-T, Ji G-Z, Zhao C-X, Yuan S-D, Ding H, Huang C, Du A-L, Wei M (1999) Self-assembling calix[4]arene [2]catenanes. Preorganization, conformation, selectivity, and efficiency. J Org Chem 64:3572-3584. doi:10.1021/jo9824100

124. Bozkurt S, Durmaz M, Yilmaz M, Sirit A (2008) Calixarenebased chiral phase-transfer catalysts derived from Cinchona alkaloids for enantioselective synthesis of $\alpha$-amino acids. Tetrahedron Asymmetr 19:618-623. doi:10.1016/j.tetasy.2008.02.006

125. Lv J, Wang X, Liu J, Zhang L, Wang Y (2006) Catalytic asymmetric epoxidation of chalcones under poly(ethylene glycol)-supported Cinchona ammonium salt catalyzed conditions. Tetrahedron Asymmetr 17:330-335. doi:10.1016/j.tetasy.2006. 01.029

126. Neumann M, Geckeler KE (1997) Telechelic polymers with azido functions as reagents for 1,3-dipolar cycloaddition reactions. React Funct Polym 33:173-184. doi:10.1016/ S1381-5148(97)00035-7 
127. Speziale AJ, Hamm PC (1956) Preparation of some new 2chloroacetamides. J Am Chem Soc 78:2556-2559. doi:10.1021/ ja01592a061

128. Park P-S, Jeong B-S, Yoo M-S, Park M-K, Jew S-S (2001) Trimeric Cinchona alkaloid phase-transfer catalyst: $\alpha, \alpha^{\prime}, \alpha^{\prime \prime}-$ tris[O(9)- allylcinchonidinium]mesitylene tribromide. Tetrahedron Lett 42:4645-4648. doi:10.1016/S0040-4039(01)00809-7

129. Siva A, Murugan E (2005) A new trimeric Cinchona alkaloid as a chiral phase-transfer catalyst for the synthesis of asymmetric $\alpha$-amino acids. Synth Stuttg 2005:2927-2933. doi:10.1055/ s-2005-872170

130. Siva A, Murugan E (2006) New trimeric Cinchona alkaloid-based quaternary ammonium salts as efficient chiral phase transfer catalysts for enantioselective synthesis of $\alpha$-amino acids. J Mol Catal A Chem 248:1-9. doi:10.1016/j.molcata.2005.11.039

131. Jayaraman S, Kumaraguru D, Arockiam J, Paulpandian S, Rajendiran B, Siva A (2014) Highly enantioselective asymmetric Michael addition reactions with new chiral multisite phase-transfer catalysts. Synlett 25:1685-1691. doi:10.1055/ s-0033-1339124

132. Maruoka K, Ooi T (2003) Enantioselective amino acid synthesis by chiral phase-transfer catalysis. Chem Rev 103:3013-3028. doi:10.1021/cr020020e

133. O'Donnell MJ, Bennett WD, Wu S (1989) The stereoselective synthesis of $\alpha$-amino acids by phase-transfer catalysis. J Am Chem Soc 111:2353-2355. doi:10.1021/ja00188a089

134. Chinchilla R, Nájera C, Ortega FJ (2008) Enantioselective cyanoformylation of aldehydes using a recyclable dimeric cinchonidine ammonium salt as an organocatalyst. Tetrahedron Asymmetr 19:265-268. doi:10.1016/j.tetasy.2008.01.011

135. Haraguchi N, Ahamed P, Parvez MM, Itsuno S (2012) Synthesis of main-chain chiral quaternary ammonium polymers for asymmetric catalysis using quaternization polymerization. Molecules 17:7569-7583. doi:10.3390/molecules17067569

136. Parvez MM, Haraguchi N, Itsuno S (2014) Synthesis of Cinchona alkaloid-derived chiral polymers by Mizoroki-Heck polymerization and their application to asymmetric catalysis. Macromolecules 47:1922-1928. doi:10.1021/ma5001018

137. Busygin I, Toukoniitty E, Sillanpää R, Murzin DY, Leino R (2005) Synthesis of chiral catalyst modifiers by hydrosilylation of cinchonidine and their application in the hydrogenation of 1-phenylpropane-1,2-dione and ethyl pyruvate on a supported $\mathrm{Pt} / \mathrm{Al}_{2} \mathrm{O}_{3}$ catalyst. Eur J Org Chem 2005:2811-2821. doi:10. 1002/ejoc.200500161

138. Khumsubdee S, Burgess K (2014) Metathesis for catalyst design: metacatalysis. Tetrahedron 70:1326-1335. doi:10.1016/ j.tet.2013.12.040

139. Braje WM, Frackenpohl J, Schrake O, Wartchow R, Beil W, Hoffmann HMR (2000) Synthesis of 10,11-didehydro Cinchona alkaloids and key derivatives. Helv Chim Acta 83:777-792. doi:10. 1002/(SICI)1522-2675(20000412)83:4

140. Kacprzak KM, Lindner W, Maier NM (2008) An improved synthesis of 10,11-didehydro Cinchona alkaloids. Chirality 20:441445. doi:10.1002/chir.20461

141. Ma B, Parkinson JL, Castle SL (2007) Novel Cinchona alkaloid derived ammonium salts as catalysts for the asymmetric synthesis of beta-hydroxy alpha-amino acids via aldol reactions. Tetrahedron Lett 48:2083-2086. doi:10.1016/j.tetlet.2007.01.132
142. Li H, Wang Y, Tang L, Deng L (2004) Highly enantioselective conjugate addition of malonate and $\beta$-ketoester to nitroalkenes: asymmetric $\mathrm{C}-\mathrm{C}$ bond formation with new bifunctional organic catalysts based on Cinchona alkaloids. J Am Chem Soc 126:99069907. doi:10.1021/ja0472811

143. Braun JV, Lemke G (1922) Ungesättigte Reste in chemischer und pharmakologischer Beziehung (III.). Ber Dtsch Chem Ges 55:3536-3559. doi:10.1002/cber.19220551022

144. Cowman AF, Deady LW, Deharo E, Desneves J, Tilley L (1997) Synthesis and activity of some antimalarial bisquinolinemethanols. Aust J Chem 50:1091-1096. doi:10.1071/C97086

145. Heidelberger M, Jacobs WA (1919) Syntheses in the Cinchona series. III. Azo dyes derived from hydrocupreine and hydrocupreidine. J Am Chem Soc 41:2131-2147. doi:10.1021/ja02233a029

146. Ochiai E, Hamana M, Noguchi Y (1946) Synthesis of cinchona base derivatives. VI. Azo dyes from diazotized 6aminohydrocinchonidine. Yakugaku Zasshi 66:48-50

147. Reichardt C, Stein J (1999) Synthesis and UV/Vis spectroscopic properties of chiral symmetrical pentamethinium cyanine dyes with $1^{\prime}, 2^{\prime}, 3^{\prime}, 4^{\prime}, 10,11$-hexahydroquinin-1'-yl and hexahydroquinidin-1'-yl end groups. Eur J Org Chem 1999:2899 2908. doi:10.1002/(SICI)1099-0690(199911)1999:11

148. Hintermann L, Schmitz M, Englert U (2007) Nucleophilic addition of organometallic reagents to Cinchona alkaloids: simple access to diverse architectures. Angew Chem Int Ed 46:51645167. doi:10.1002/anie.200701341

149. Corey EJ, Noe MC (1993) Rigid and highly enantioselective catalyst for the dihydroxylation of olefins using osmium tetraoxide clarifies the origin of enantiospecificity. J Am Chem Soc 115:12579-12580. doi:10.1021/ja00079a045

150. Lohray BB, Singh SK, Bhushan V (2002) A mechanistically designed Cinchona alkaloid ligand in the osmium catalyzed asymmetric dihydroxylation of alkenes. Indian J Chem B 41: 1226

151. Corey EJ, Noe MC, Sarshar S (1994) X-ray crystallographic studies provide additional evidence that an enzyme-like binding pocket is crucial to the enantioselective dihydroxylation of olefins by $\mathrm{OsO}_{4}$-bis-Cinchona alkaloid complexes. Tetrahedron Lett 35:2861-2864. doi:10.1016/S0040-4039(00)76644-5

152. Rowan SJ, Brady PA, Sanders JKM (1996) Structure-directed synthesis under thermodynamic control: macrocyclic trimers from Cinchona alkaloids. Angew Chem Int Ed 35:2143-2145. doi:10. 1002/anie.199621431

153. Rowan SJ, Reynolds DJ, Sanders JKM (1999) Effects of shape on thermodynamic cyclizations of Cinchona alkaloids. J Org Chem 64:5804-5814. doi:10.1021/jo982496x

154. Rowan SJ, Hamilton DG, Brady PA, Sanders JKM (1997) Automated recognition, sorting, and covalent self-assembly by predisposed building blocks in a mixture. J Am Chem Soc 119:25782579. doi:10.1021/ja963320k

155. Jankowski R, Joseph D, Cavé C, Dumas F, Ourevitch M, Mahuteau J, Morgant G, d'Angelo J (2003) Synthetic approaches to Cinchona alkaloids: the C-8/C-9 disconnection strategy. Tetrahedron Lett 44:4187-4190. doi:10.1016/s0040-4039(03)00926-2 